\title{
Supramolecular Photochemistry Concepts Highlighted with Select Examples
}

\author{
V. Ramamurthy* and Barnali Mondal \\ Department of Chemistry, University of Miami, Coral Gables, FL, 33146, USA \\ Email: murthy1@ @iami.edu; Voice: 1-305-284 1534
}

\begin{abstract}
'Supramolecular Photochemistry' (SP) deals with a study of the properties of molecules in their excited states where the medium plays a significant role. While 'Molecular Photochemistry' (MP) deals with studies in isotropic solution, the SP deals with reactant molecules that interact weakly with their surroundings. The surroundings in general are highly organized assemblies such as crystals, liquid crystals, micelles, hostguest structures etc. The behavior of exited molecules in SP unlike in isotropic solution is controlled not only by their inherent electronic and steric properties but also by the immediate surroundings. The weak interactions that control the chemistry include van der Walls, hydrophobic, $\mathrm{C}-\mathrm{H}---\pi, \pi---\pi$ and several types of hydrogen bonds. In this review the uniqueness of SP compared to MP is highlighted with examples chosen from reactions in crystals, micelles and host-guest assemblies. In spite of distinctly different structures (crystals, micelles etc.) the influence of the medium could be understood on the basis of a model developed by G. M. J. Schmidt for photoreactions in crystals. The principles of reaction cavity model are briefly outlined in this review. There are a few important features that are specific to SP. For example, highly reactive molecules and intermediates could be stabilized in a confined environment; they enable phosphorescence to be observed at room temperature and favor chiral induction in photochemical reactions. Using such examples the uniqueness of SP is highlighted. The future of SP depends on developing efficient and unique catalytic photoreactions using easily available reaction 'containers'. In addition, their value in artificial photosynthesis should be established for SP to occupy a center stage in the future.
\end{abstract}

Key words: Supramolecular chemistry, Reaction cavity, Solid state photochemistry, Micellar chemistry, Chiral photochemistry, Host-guest chemistry, Cavitands. 


\section{Introduction}

The momentous term, 'supramolecule' coined by J. M. Lehn, the Nobel Prize awarded to Pederson, Lehn and Cram "for their development and use of molecules with structure-specific interactions of high selectivity" in 1978 and Lehn's book [1] attracted a number of researchers to the topic of supramolecular photochemistry that has already been of sporadic interest for over a century. Thermal and photoreactions carried out in the preferred solid state during late 1800 and early 1900 were replaced by 'liquid' by mid1900. Reaction controls that were largely achieved by varying the reagent, temperature, pressure and solvent were difficult in light activated reactions. In this context the strategy of controlling photoreactions by confinement and through weak interactions has been extensively investigated since 1970s. The several reviews and monographs emphasizing the value of highly ordered media to manipulate photoreactions [2-13] published during 1975-1991 were quite pivotal to the development of the field.

Under the broad canopy 'supramolecular', several media have been explored to carry out photoreactions. These include crystals, liquid crystals, micelles, LB films, internal and external surfaces of solids (silica, clay and zeolites), polymer films, hostguest complexes in solution and solid state. Of the various assemblies, crystals have received considerable attention and numerous reviews have been written; readers are directed to these for in depth understanding of the topic.[14-110] Despite the crystal's ability to pre-organize molecules toward a particular product, reliable predictability of crystal structure and packing remains unsatisfactory. Tremendous experimental and technological progress in X-ray crystal structure solution has contributed to the renaissance of the field of solid-state photochemistry. Difficulty in handling large amounts of samples for preparative scale irradiations of liquid crystals, polymer films and LB films have curtailed their becoming a medium of choice for manipulating photoreactions. Reviews should be consulted to comprehend the opportunities and limitations involved in the use of these media.[3, 7, 111-117] Amongst the solid surfaces considerable control on excited state reactions have been achieved in zeolites which are easier to handle and commercially available at modest cost; even large pore zeolites (MCMs) can be easily synthesized. Availability of zeolites with varying internal structures and dimensions has attracted a number of groups to explore them as reaction 
media. For in depth coverage of photochemistry within zeolites readers are directed to reviews on this topic.[118-147] One and two-dimensional surfaces such as silica, clay and $\mathrm{Zr}$ phosphate have also been examined as reaction media. Better predictable confinement of zeolites than silica, clay and $\mathrm{Zr}$ phosphate is noteworthy. Several reviews on photochemistry and photophysics of organic molecules on solid surfaces are available for consultation.[8, 142, 148-163] Lastly, organic solid-state host-guest complexes have also been examined to probe the role of hosts on the excited state behavior of guests. Examined hosts include cyclodextrins, urea, deoxycholic acid, Dianin's compound, trisorthothymotide etc. Despite initial promising examples, progress on this topic has been limited.[164-166] The idea of employing organic hosts to control photoreactions has been revived and a recently published review on this topic should be of considerable interest to the readers.[165]

Impressive examples have been reported in crystals, zeolites, micelles, and hostguest complexes in aqueous solution that have shown considerable promise among the various confined media. Concepts involved in supramolecular photochemistry will be illustrated utilizing some of these examples. Micelles, zeolites and host-guest complexes in aqueous solution have been demonstrated to enhance fluorescence, phosphorescence, and emission from excimers and provide selectivity during photodimerization of olefins, recombination of singlet and triplet radical pairs resulting from photo-Fries and Norrish type I and type II reactions of carbonyl compounds and geometric isomerization of olefins. Classic studies on magnetic isotope and magnetic field effects on radical recombination reactions in micellar media conducted by Turro[167] stimulated further interest in supramolecular photochemistry. This review highlights examples of zeolites and cyclodextrins that have played an important role in the development of 'chiral photochemistry', yet another topic of importance specific to confined media. Readers are directed to the numerous published reviews on micelles, cyclodextrins, cucurbiturils, Pdnanocage and capusles [4, 168-197] and recent books for additional information.[198200] 


\section{Organization}

This review focuses mainly on highlighting concepts. For detailed reports on this topic readers are directed to reviews and monographs cited in reference section. In Section III, the role of confined media on reactants in the ground and excited states and on intermediates and products are brought to light with chosen examples from zeolites, micelles and host-guest complexes in solution. In Section IV, a model enabling an understanding of photoreactions in various confined media is presented with supporting examples. Section V highlights two phenomena, namely room temperature phosphorescence and asymmetric induction in photochemical reactions in which supramolecular structures have played an important role. These selected examples should help the readers appreciate the value and potential of supramolecular assemblies in bringing out the hidden side of reactive molecules.

\section{Controlling photoreactions through the influence of media on reactants, intermediates and products.}

As illustrated in Scheme 1 photochemical reactions are comprised of four stages, reactant in the ground state $(R)$, reactant in the excited state $(* R)$, intermediate (I) and product in the ground state (P).[201] Organized media could control product distributions by influencing the reaction at any or all stages. Examples of these are discussed in the following sections. For easy conceptualization, the influence of organized media on photoreactions is discussed under four sections, $\mathrm{R}, * \mathrm{R}, \mathrm{I}$ and $\mathrm{P}$.

\section{IIIa. Effect of organized media on reactants in the ground state $(R)$}

For an olefin with two substituents (Scheme 2) geometric isomerization from $\mathrm{S}_{1}$ and $\mathrm{T}_{1}$ in solution is typical.[201] At higher concentrations however, dimerization is likely and of the several isomeric dimers possible in principle, single dimer can be targeted by preventing geometric isomerization and by pre-organization of olefin molecules and their continued preservation during the excited state lifetime. The latter conditions can be achieved with the help of organized media. Examples of solid-state photodimerization provided below illustrate the concept. 
Trans-cinnamic acid yields the $c i$ is isomer upon irradiation in solution. On the other hand, irradiation of the crystalline material results in [2+2] dimerization (Scheme 3) $[17,18]$ and more importantly a single one from arrested isomerization due to increased barrier for the process in the excited state. How does the crystalline state favor a single dimer? Cinnamic acids crystallize in three polymorphic forms, namely, $\alpha, \beta$ and $\gamma$ determining their photochemical behavior. In the $\beta$-type packing with the molecules separated by a short repeat distance of 3.9-4.1 $\AA$, the neighboring molecules above the stack being translationally equivalent and with considerable face to face overlap as shown for a substituted cinnamic acid in Figure 1. All cinnamic acids, which crystallize thus react photochemically to give products of the same stereochemistry (mirror symmetric dimers). In the $\alpha$-type the double bond of a molecule in one stack overlaps with that of a centrosymmetrically related molecule in an adjacent stack. The distance between the equivalent double bonds is greater than $5.5 \AA$, but that between the overlapping double bonds is less than $4.2 \AA$. This type of crystal produces centrosymmetric dimers upon irradiation. $\alpha$-Type packing arrangement of a substituted cinnamic acid is shown in Figure 2. Crystals with the $\gamma$-type structure have the adjacent molecules offset such that the reactive double bonds do not overlap, and have large distance (4.7-5.1 $\AA$ ) between them and are photostable.

In the example above, pre-organization is achieved during crystallization. With the less understood knowledge of factors controlling crystal packing that prevents extrapolating information from one molecule to another, a strategy known as 'intermolecular templation' is utilized to orient molecules. This concept illustrated with an example below shows how molecules could be preorganized with the help of a carefully chosen host molecule. In this strategy utilizing a carefully chosen template the packing of the template/host molecules in the crystalline state is tailored to enable the potentially reactive guest molecules to pack in a manner facilitating photodimerization. trans-1,2-Bis(4-pyridyl)ethylene (4, 4'-BPE) upon irradiation in solution undergoes cistrans isomerization. Irradiation of its crystals does not give any products. Scrutiny of the crystal structure reveals that the molecules crystallize in a layered structure in which olefins of neighboring molecules are separated by more than $5.7 \AA$ ( $\gamma$-type packing; (Figure 3), a distance too large for dimerization to occur. However, this molecule can be 
engineered to dimerize in the crystalline state when co-crystallized in the presence of 1,3dihydroxybenzene or thiourea (Figure 3). [55, 56, 202] For example, contrary to the geometric isomerization observed on irradiation of a mixture of 1,3-dihydroxybenzene and trans-1,2-bis(4-pyridyl)ethylene in solution a single photodimer was obtained in quantitative yield when the mixture was irradiated in the crystalline state. The hydrogen bonding between 1,3-dihydroxybenzene and trans-1,2-bis(4-pyridyl)ethylene keeps the olefins parallel to each other and within $4.2 \AA$, to facilitate the dimerization process.

Thiourea is another molecule that functioned as a template by the same principle in our hands.[202] We noted that the anti $\mathrm{N}-\mathrm{H}$ atoms were free to interact with a hydrogen bond acceptor such as pyridyl unit when thiourea self-assembled into tapes via $\mathrm{N}-\mathrm{H} \cdots \mathrm{S}$ hydrogen bonded dimer synthon using syn $\mathrm{N}-\mathrm{H}$ atoms from a published structure. The co-crystal engineering strategy yielded 1:1 co-crystals of thiourea with trans-1,2-bis(4-pyridyl)ethylene. The parallel alignment of the alkene double bonds of the alkene pairs and within $4.15 \AA$ A resulted in syn dimer in quantitative yield on photoexcitation (Scheme 4, Figure 4a). Surprisingly thiourea forms 2:1 co-crystals with trans-1-(2-pyridyl)-2-(4-pyridyl)ethylene where each thiourea molecule interacts with only one pyridyl nitrogen via an acceptor bifurcated $(\mathrm{N}-\mathrm{H})_{2 \text { (thiourea) }}{ }^{\cdots} \mathrm{N}_{(\text {pyridyl) }}$ hydrogen bond (Figure 4b). The reactant molecules here preorganized in head to tail fashion with the double bonds separated by $3.95 \AA$ and gave anti $\mathrm{H}$-T dimer in $100 \%$ yield upon photoexcitation (Scheme 4). An inspection of this co-crystal structure hinted at the auxiliary role of the thiourea molecule at 2-pyridyl position and that the interdigitation 4stilbazole class of molecules would form a similar packing in 1:1 co-crystal with thiourea to direct them to align in head to tail fashion.

An experimental investigation of the above hypothesis revealed the versatility of thiourea template (Figure 5). Six stilbazole derivatives aligned in head to tail orientation in their 1:1 thiourea cocrystals successfully yielded anti $\mathrm{H}$-T dimer products in about 95\% by photoirradiation (Scheme 4). In 1:1 cocrystals of thiourea and cyanostilbazole the cyano group accepts hydrogen bond similar to pyridine nitrogen in 1:1 cocrystals of thiourea and 4,4'-BPE and forms a similar packing. Photoexcitation of these cocrystals where the cyanostilbazole molecules preorganized in head to head fashion leads to the formation of syn $\mathrm{HH}$ dimer with $100 \%$ yield. Achieving similar packing patterns with 
different molecules in a family of compounds in solid-state remains a challenge since very small changes in the geometrical and chemical properties of a functional group could lead to entirely different supramolecular arrangements. We were able to successfully employ thiourea as a template for nine different pyridyl olefin compounds to yield a single regio-isomer with $>95 \%$ yield and have recently published the results.[203]

Photodimerization of coumarin in the solid state provides the most impressive product control achieved. Excitation of coumarin dissolved in organic solvents yields several dimers while dimerization does not occur in crystalline state due to unfavorable packing. Coumarin can however be steered to yield a single optically active anti-headhead dimer when crystallized within an optically active host (Scheme 5).[65, 66] The host not only brings the two molecules within the reactive distance but also aligns them in a single pro-chiral form (Figure 6). Such a control has not been achieved in solution.

Above remarkable pre-orientation of reactants during cycloaddition reactions is not restricted to solid state. It can occur in aqueous solution in microheterogeneous medium. Micellar surfaces and water-soluble hosts such as cyclodextrins, cucurbutrils and Pd host can prealign the reactants prior to excitation. In these organized media the boundary of the 'reaction cavity' may separate two phases of very different properties. For example the boundary of a micelle separates the non-polar hydrocarbon interior from the polar aqueous exterior (Figure 7). Similarly cyclodextrins, cucurbutrils and Pd host provide both a non-polar interior and a polar aqueous exterior region (Figure 7). These interfacial boundaries allowing one to simultaneously expose a reactant molecule to two phases with different properties work well with reactant molecules with both hydrophobic and hydrophilic components.

For example, a molecule with a hydrophobic nonpolar hydrocarbon part and hydrophilic polar functional groups such as $\mathrm{OH}, \mathrm{COOH}$ and $\mathrm{CO}$ when solubilized in water with the help of a micelle or a water-soluble host will adopt a specific arrangement with the hydrophobic part in the hydrocarbon like phase and the hydrophilic part exposed to the aqueous phase of the micelle. The alignment of the reactants at the interface might restrict the randomness in their orientation and could hence lead to regioselectivity during bimolecular reactions. For example, photocycloaddition of 3-n-butylcyclopentanone in 
the presence of heptenyl acetate in organic solvents gives a single adduct B (Scheme 6).[204-206] Irradiation of these two compounds solubilized in a potassium decanoate micelle yields a mixture of two adducts $\mathbf{A}$ and $\mathbf{B}$ in which the $\mathbf{A}$ is the major product and consistent with the notion that a micellar interface helps orient the reactant molecules (Figure 8).

The doughnut shaped cyclodextrins (CDs) are cyclic oligosaccharides consisting of six to eight glucose units ( $\alpha-, \beta$ - and $\gamma$-CDs, respectively) with a hydrophobic inner cavity and a hydrophilic outside. These cavities with internal diameters varying from 4.7 to $8.3 \AA$, and with an almost same depth $(7.8 \AA)$ allow formation of inclusion complexes with a variety of guest molecules. Complexation between CDs and substrates in solution has been successfully exploited to induce selectivity in some unimolecular and bimolecular photochemical reactions. Tamaki and co-workers' first exploration of the utility of the CD-cavity in effecting regio- and stereo-selective dimerization of guest molecules such as anthracene-2-sulfonate and anthracene-2-carboxylate [207] has been followed by several groups' exploration of photodimerization of acenaphthylene, coumarin, stilbene derivatives, trans-2-styrylpyridine and bispyridylethylenes included within $\gamma$-CD (Scheme 7).[208-211] The dimer formed in majority of these examples could be rationalized on the basis of pre-orientation due to hydrophobic-hydrophilic alignment at the interface and confinement by the CD cavity.

Cucurbiturils with reaction cavities similar to cyclodextrins form water-soluble host-guest complexes. Kim and coworkers first demonstrated that the cavity of $\mathrm{CB}[8]$ can accommodate and orient the olefinic bonds of diaminostilbene hydrochloride to high yields of syn dimer (Scheme 8).[212] The selective formation of syn dimer within the host was proposed to be due to the arrangement of the olefins within the cavity favoring the formation of the syn dimer.

A few years ago, our group demonstrated that alkenes photostable in the solid state dimerize within cucurbiturils.[211, 213-215] The three cinnamic acids listed in Scheme 10 do not react in solid state and geometrically isomerize in solution. However, excitation of these included in cucurbituril [8] in water readily yielded syn-H-H dimer exclusively. While the two reactive olefin molecules could be brought together within the confined cavity, it is the weak intermolecular forces that orient them in a proper geometry 
for dimerization. We believe that the inclusion of the two olefins in a head-head fashion is a combination of $\pi---\pi$, hydrogen bonding and hydrophobic interactions. While the former two facilitate orientation, the later favors inclusion. In the head-head arrangement the two acid groups face the water and the two-phenyl groups would be forced to stay parallel favoring $\pi---\pi \square$ interaction (Figure 9).

Owing to space limitations we refer the readers to the publications by the groups of Sivaguru and $\mathrm{Li}-\mathrm{Zhu} \mathrm{Wu}$ on the use of cucurbiturils as templates in aqueous solution for photodimerization reactions (Schemes 8 and 9).[178-180, 216-221] In addition to cyclodextrins, cucurbiturils and micelles, an organometallic host synthesized by Fujita also used as a host in water to orient, confine and dimerize molecules.[181-184, 222-231] Most of the examples reported by Fujita that are listed in Scheme 11, 12 and 13 seem to rely on the confinement provided by the host. Two examples namely cinnamic acids and coumarins provided by our group highlight the importance of hydrophobic-hydrophilic interface during the alignment of olefins within these cages.

The Pd nanocage shown in Figure 7 readily included the cinnamic acids and coumarins listed in Schemes 14 and 15 and formed water-soluble complexes which upon irradiation gave a single, corresponding syn-head-head dimer clearly attributable to the confinement and templation afforded by the host.[232,233] The hydrophobic effect alone can't be responsible for such selectivity. While it could force the cinnamic acid and coumarin molecules to stay within the cage it is likely that the interface between hydrophobic exterior and hydrophilic interior aligned the polar groups at the interface towards water and favored syn-head-head dimers (Figure 10).

In the photochemistry of various olefins discussed above control is exerted by the organized medium where the reactant molecules are pre-organized prior to excitation. In the organized medium's confined reaction cavity the restricted mobility of the reactants in the ground as well as in the excited state reduces their entropy and the free energy barrier for competing reactions (e.g., isomerization) is increased in the excited state. Thus in the examples discussed above the media has an influence on the reactant(s) in their ground as well as in excited states. Thus one can't separate the influence of the medium on $\mathrm{R}$ and $\mathrm{R}^{*}$, it has on both. 


\section{IIIb Effect of organized media on reactant in the excited state ( $\left.\mathbf{R}^{*}\right)$}

The example in this category deals with intramolecular $\gamma$-hydrogen abstraction reaction in ketones shown in Scheme 16. In solution the excited carbonyl will abstract the both prochiral hydrogen atoms in these molecules with equal efficiency leading to $1: 1$ mixture of the two optical isomers of the product cyclobutanol.[28, 31, 33-35] Thus the two pathways have identical barriers. However, irradiation of crystals obtained from the salts of the corresponding acids and a chiral amine results in cyclobutanol in fairly high enantiomeric excess (ee) proving a preference for one of the hydrogen atoms. The main effects of the medium in this reaction is the ordering of the reactants in a single homochiral conformation that allows selective abstraction of one of the two available enantiotopic hydrogens. The two prochiral hydrogens are no longer identical and so are the barriers for abstraction of the two hydrogens. The X-ray crystal structure shown for a ketone in Figure 11 clearly shows that one of the hydrogens is closer to the carbonyl chromophore. The chiral packing of the crystal ensured by the base ephedrine provides identical arrangement of all molecules in the crystal with the same prochiral hydrogen closer to the carbonyl chromophore. In this strategy termed the 'ionic chiral auxiliary approach' though the ground state molecule is entropically prepared to form a single chiral product, the effect is felt during the reaction of $* \mathrm{R}$. In the supramolecular structure the activation barrier for one of the two possible reaction paths in the excited state is lower.

\section{IIIc Effect of organized media on reaction intermediates (I)}

In the examples discussed above the reaction medium influenced the photochemical outcome by lowering the entropy (restricting the freedom) of R and * $\mathrm{R}$. Organized media could likewise control the mobility (entropic control) of the reactive intermediates (I) generated in a photochemical reaction. The best examples of these are found in photofragmentation reactions of carbonyl compounds.[201] As illustrated in Scheme 17 excitation of 3-(4-methylphenyl)-1-phenylacetone (MeDBK) results in a radical pair intermediate yielding at least three products in a statistical ratio of 1:2:1. An organized medium such as micelles could influence the diffusional mobility of the radical pair A and B and thereby affect product distribution. The reaction cavity of a micelle is made up of a hydrophobic core and a hydrophilic exterior. A highly polar boundary 
separates the hydrophobic core from the aqueous exterior. Such a boundary provides features unique to these media absent in isotropic solution. Hindrance of the translational motion of a guest present within the reaction cavity by the well-defined boundary is a feature common to all heterogeneously organized media such as cyclodextrins, mono and bilayers, host-guest complexes and vesicles in water.

As shown in Scheme 17 photolysis of 3-(4-methylphenyl)-1-phenylacetone (MeDBK) results in a 1:2:1 mixture of three products $\mathrm{AA}, \mathrm{BB}$, and $\mathrm{AB}$. Photolysis of the same molecule solubilized in a micelle (hexadecyltrimethylammonium chloride) gives $\mathrm{AB}$ as the major product.[167, 234] The distinctive change in product distribution occurring at and above the critical micelle concentration $(\mathrm{cmc})$ (the minimum concentration at which a micelle is formed) due to the restricted mobility of the radical pair within the micellar structure is consistent with the radical intermediates trapped in a small hydrophobic reaction cavity. How does the reaction cavity present in an isotropic solution differ from that in a micelle? One of the differences lies in the size of the reaction cavity. In principle during the time required for a reaction, the entire volume of the solution in the flask is accessible to the excited molecule in an isotropic medium while it may be confined to a single micelle in a micellar medium. The space available for the reacting molecule has thus shrunk. In isotropic solution, the A and B radicals generated from DBK, experience unrestricted translational mobility (depending on the viscosity of the solution) that enables them to rapidly diffuse apart and find partners based only on a statistical probability (AA, AB, BB). On the other hand, in a micellar medium, at low occupancy levels, the single A and B radical pair likely to be generated within a single micelle with movement restricted by the hydrophobic-hydrophilic boundary is forced to find a partner within the same micelle $(A B)$, to result in their coupling.

The concomitant increase of the cage effect (see Figure 12 for definition) with the number of methyl groups is consistent with the hydrophobicity of the secondary radical pair (A and B). For example, the cage effect within hexadecyl trimethyl ammonium chloride micelle for DBK is 31\% whereas that for 4,4'-di-tert-butyl DBK is 95\%[235]. The results of photolysis of MeDBK in micelles with chain lengths varying between 6 - 14 as shown in Figure 13 suggests an inverse relationship between the rate 
of radical escape from a micellar cage and micelle size (larger the micelle, greater the radical pair retention). The estimated exit rates of the benzyl radical from micelles of different sizes (sodium decyl sulfate: $2.7 \times 10^{6} \mathrm{~s}^{-1}$; sodium dodecyl sulfate: $1.8 \times 10^{6} \mathrm{~s}^{-1}$ and sodium tetradecyl sulfate: $1.2 \times 10^{6} \mathrm{~s}^{-1}$ ) correlate well with the observed trends in the cage effect. Thus, the extent to which the radicals (A and B) can escape the micellar reaction cavity as estimated experimentally by measuring the cage effect depends upon both the size of the reaction cavity (micellar size) and the hydrophobicity of the radical pair. The more hydrophobic the radical, less it would venture outside the micellar reaction cavity. The larger the micelle, the less the radical pair escapes the reaction cavity. Thus, the cage effect, measuring the extent to which the radicals (A and B) can escape the micellar reaction cavity depends upon both the size of the reaction cavity (micellar size) and the hydrophobicity of the radical pair.

The difference in the nature of the restriction provided by an isotropic solvent and a micelle is nicely demonstrated by the isotope enrichment observed during the photolysis of DBK. This process can be understood using the detailed mechanism for cleavage process presented in Scheme 18. $\alpha$-Cleavage of the triplet excited DBK results in a triplet geminate primary radical pair $\left(\mathrm{C}_{6} \mathrm{H}_{5} \mathrm{CH}_{2}{ }^{\circ}\right.$ and $\left.\mathrm{C}_{6} \mathrm{H}_{5} \mathrm{CH}_{2} \mathrm{CO}^{\circ} ; \mathrm{PRP}^{3}\right)$. This initial radical pair loses $\mathrm{CO}$ to generate the secondary radical pair $\left(2 \mathrm{C}_{6} \mathrm{H}_{5} \mathrm{CH}_{2}{ }^{\circ} ; \mathrm{SRP}\right)$. The two benzylic SRP combines to yield the final product, 1,2-diphenylethane. An option, unavailable in isotropic solution to the $\mathrm{PRP}^{3}$, becomes available within a micellar reaction cavity. If the $\mathrm{PRP}^{3}$ is constrained long enough to allow intersystem crossing to the singlet state $\mathrm{PRP}^{1}$, it can recombine to yield the starting ketone with or without rearrangement. The latter would yield 1-phenyl-4-methylacetophenone (PMAP). In a micelle, the triplet-to-singlet intersystem crossing of $\mathrm{PRP}^{3}$ competes with the decarbonylation process and the cage escape. The rate of decarbonylation of phenylacetyl radical has been established to be $6.4 \times 10^{6} \mathrm{~s}^{-1}$ at room temperature, and the rate of cage escape from the micelle has been estimated to be in the range of $10^{6} \mathrm{~s}^{-1}$. Intersystem crossing facilitated by the hyperfine interaction between ${ }^{13} \mathrm{C}$ nuclei at the carbonyl carbon (or the benzylic position) and the unpaired electron spin could occur in the microsecond time scale. Since ${ }^{12} \mathrm{C}$ nuclei do not enhance intersystem crossing, the competition between decarbonylation and intersystem crossing of $\mathrm{PRP}^{3}$ to $\mathrm{PRP}^{1}$ can only 
occur in molecules containing $\mathrm{C}^{13}$ nuclei. Based on the established mechanism for the nuclear hyperfine-induced intersystem crossing one would expect a higher yield of PRP ${ }^{1}$ from molecules containing ${ }^{13} \mathrm{C}$ nuclei and decarbonylation from those with ${ }^{12} \mathrm{C}$ nuclei. This selective process would be expected to yield ${ }^{13} \mathrm{C}$ enriched DBK and PMAP. This has been demonstrated by photolysis of DBK that is $48 \%$ enriched in ${ }^{13} \mathrm{C}$ at the carbonyl carbon.[236-239] After 90\% photolysis the CO carbon of the remaining DBK has $62 \%$

${ }^{13} \mathrm{C}$ as compared to the $48 \%$ at the start. Similar enrichment in PMAP was also noted. The absence of such effects in homogeneous solutions is due to the lifetime of the solvent cage that is much shorter $\left(<10^{-10} \mathrm{~s}\right)$ than that of a micelle $\left(\sim 10^{-6} \mathrm{~s}\right)$. Within the short time of the solvent cage nuclear hyperfine coupling will not be able to induce intersystem crossing of $\mathrm{PRP}^{3}$ to form $\mathrm{PRP}^{1}$. The above remarkable results reported by Turro and coworkers prompted considerable interest in supramolecular photochemistry.[167, 192, 197]

\section{IIId. Effect of organized media on photoproducts (P)}

Photochemical reactions are often capable of producing highly energy rich and less stable products and intermediates. For example, molecules such as cyclobutadiene, benzyne, benzocyclopropanone etc. generally having a fleeting existence in solution at room temperature when generated within a confined space (organized structure) in solution, are stable for hours to days. In this regard, a class of organized host systems known as hemicarcerands has been very useful for this purpose (Scheme 19).[185-187]

Cyclobutadiene while stable only in inert gas matrices at extremely low temperatures when generated upon irradiation of $\alpha$-pyrone trapped in a hemicarcerand cyclobutadiene (Scheme 20) under oxygen free condition is indefinitely stable at room temperature. Stabilization of cyclobutadiene at room temperature in a confined container is a masterpiece in the area of supramolecular photochemistry.[240, 241]

Photolysis (>400 nm) of benzocyclobutenedione trapped within a hemicarcerand yields the highly strained benzocyclopropanone (Scheme 21). Benzocyclopropanone while normally unstable at room temperature when trapped hemicarcerand can even be subjected to X-ray crystallographic studies. Upon irradiation (>300 nm) this trapped molecule eliminates carbon monoxide to yield benzyne which is stable for several hours 
at $-78^{\circ} \mathrm{C}$ in this condition while stable only at cryogenic temperature in inert matrices.[242]

Benzyl radicals when generated in solution with lifetimes less than a microsecond when generated by photolysis of dibenzyl ketones trapped in cyclodextrins or zeolites, in solid state, have lifetime of days. For example, phenylethyl radical generated by photolysis of $\alpha, \alpha^{\prime}$-dimethyldibenzylketone included in $\beta$-cyclodextrin is stable for a minimum of three days.[243] The lifetime of diphenylmethyl radical in solution being controlled only by diffusion limited radical-radical coupling has a lifetime in the order of micro to nanoseconds. The same radical generated in the channels of ZSM-5 type zeolites by photolysis of tetraphenyl acetone is stable for weeks (Scheme 22).

In this regard, one should note that a number of carbenes and nitrenes have been stabilized in confined environments and their photochemical and photophysical properties investigated (Scheme 22). [144, 244-254] In this context one should note that radical cations of stilbene and other polyenes have been stabilized for even months in the channels of ZSM-5 zeolites. 1,n-Diphenyl alkene cation radicals, with normal lifetimes of nanoseconds, remain stable for months in the channels of ZSM-5.[255, 256] Their lengthy lifetimes enables their absorption and emission recordings at room temperature (Figure 14).

From the examples above, it should be clear that the concept of time within organized media is different from that in isotropic solution. What we normally consider as fleeting and short-lived become stable within organized media. Under such conditions, characterizing the excited state properties of radicals, radical ions, carbocations and reactive species such as cyclobutadiene requires no special time resolved techniques. In organized media, intermediates and products, which based on behavior in solution would be defined to be highly reactive, become 'tame' with lifetimes several orders longer than that in solution. That the organized medium stabilizes the products by restricting their mobility and accessibility by other reactants is noteworthy.

\section{A model for conceptualizing photoreactions in organized media: Reaction cavity model}


From the above discussion it is clear that reaction environment provided by an organized medium has unique features mimicking those of proteins. While crystals (and zeolites) provide inflexible reaction cavities a whole spectrum of organized media (e.g., micelles, host-guest complexes, monolayers and bilayers, liquid crystals etc.) allow different degrees of freedom to the reactant molecules. These systems demonstrate the clever usage of favorable entropy an important requisite to natural systems. An understanding of the unique features of individual organized media should enable appropriate choice of reaction medium to achieve the desired selectivity. In this section we discuss some of the common characteristics of the reaction cavities of the various organized media.[20, 41, 257-259]

A chemical reaction can be viewed as a molecular shape changing topological phenomenon. Occurrence of a particular reaction will depend upon whether the product would fit within the reactant occupied 'reaction cavity' (Figure 15). The reaction cavity by definition is the space occupied by the reactant molecules that includes the void space surrounding them (Figure 16). The reaction cavity wall is made up of molecules adjacent to the reacting molecules. Since the boundaries of a reaction cavity are undefined in an isotropic solution, size matching of the reactant, products and the reaction cavity is not important in this medium. On the other hand, when the reaction cavity has a well-defined boundary, as in most organized assemblies (especially in solid state and host-guest complexes), size matching between the reactant and the product can become important and occasionally may even be the sole factor controlling the feasibility of a reaction.

Consider for example conversion of reactant $\mathrm{R}$ into products $\mathrm{P} 1$ and $\mathrm{P} 2$ of different shapes (Figure 17). In gas phase both products would be formed. In isotropic solution fast relaxation of solvent molecules would accommodate the shape changes from $\mathrm{R}$ to both $\mathrm{P} 1$ and $\mathrm{P} 2$. On the other hand in an organized medium where the reaction cavity is time independent (crystals, host guest complexes in solid state and solution, zeolites and clay) only one of the two products would be formed. The free volume within a reaction cavity is an important parameter whose shape, size, location, directionality and dynamics control in large part the extent to which the medium influences a photoreaction. 


\section{IVa. Importance of free volume}

In order to accommodate the shape changes as the reactants transform to products, the reaction cavity must contain a certain amount of free volume. All condensed media, with the possible exception of perfect crystals of monatomic solids, contain free volume. Normally, the free volume in a liquid state is greater than in the crystalline state of the same substance, as evidenced by the decrease in density on melting of most solids except ice. In isotropic liquids, the free volume is highly mobile by virtue of the motions (translation, rotation, vibration, internal rotation, etc.) of the constituent molecules. In organized media, the free volume may be essentially immobile, as in crystals, or have mobility ranging from that found in crystals to that of isotropic liquids. Since the surfaces of silica, the inter planar regions of clay, and the interiors of zeolites, possess timeindependent structures similar to those of crystals (whose relaxation times are much greater than that necessary to transform a reactant molecule to its products), the free volume needed to accommodate the shape changes during the course of a reaction must be intrinsic to the reaction cavity. The "stiff" reaction cavities of such media may be characterized as being 'hard' (Figure 18).

Distinct differences exist between the rigidly organized structures discussed above and media such as micelles, microemulsions, molecular aggregates, and liquid crystals. In the latter group, the guest reaction cavities may contain minimal intrinsic free volume at the time of photoexcitation. However, since the molecules constituting these organized assemblies are mobile, the reaction cavity can respond to shape changes as the reaction proceeds. The extent and ease with which each medium can accommodate shape changes will determine the selectivity that can be obtained. The cavities of these media with 'flexible' walls may be characterized as being 'soft' (Figure 18). The ease of a medium's response to shape changes during the course of a reaction depends on the extent of co-operative motions involving guest and host molecules. For a guest molecule to react in the restrictive host's environment, requisite adjustments must be made by the medium within the time frame determined by the rate limiting paths leading to the transition state(s). No reaction will occur if the host medium fails to respond in a 'timely' fashion. 
The importance of free volume is nowhere more evident than in the photoreactions of crystalline molecules. Irradiation of crystalline 7-chlorocoumarin yields a single dimer (syn head-to-head) (Figure 19).[15, 16, 260, 261] The packing arrangement shown in Figure 19 reveals two potentially reactive pairs of 7chlorocoumarin molecules in a unit cell; the translationally related pair has a center-tocenter distance of $4.54 \AA$ (favored to yield the syn head-to-head dimer); the other centrosymmetrically related pair has a center-to-center distance of $4.12 \AA$ (favored to yield the anti head-to-tail dimer). Despite the favorable arrangement of the latter pair the dimer is obtained from the former pair only. Lattice energy calculations reveal a much higher relative increase if the centrosymmetrically related pair were to react. In other words, the larger free volume around the translationally related pair allows dimerization of this less favored pair while despite the centrosymmetrically related pair whose double bonds are initially closer the lack of free volume in the most topochemically favored pair leads to no reaction.

A comparison of the solid state photochemistry of two crystals, 7methoxycoumarin and methyl $m$-bromocinnamate, further exemplifies the importance of free volume near the reaction site (Figures 20 and 21).[262, 263] Photodimerization occurs in crystals of 7-methoxycoumarin to give the syn head-to-tail isomer despite the reactive double bonds being rotated by $65^{\circ}$ with respect to each other and a center-tocenter double-bond distance of $3.83 \AA$ (Figure 20). On the other hand, methyl $m$ bromocinnamate, with the distance between the centers of adjacent double bonds is 3.93 $\AA$, rotated and makes an angle of $28^{\circ}$ when projected down the line joining the centers of the bonds does not undergo photochemical dimerization in the crystalline state (Figure 21). Between these two poorly oriented molecules one yields a dimer while the other does not. Based on the estimated increase in lattice energy $(200 \mathrm{kcal} / \mathrm{mol})$ upon reorientation of the reactive pairs in the crystal lattice 7-methoxycoumarin's was found to be roughly the same order of magnitude as for many photoreactive crystals with favorably oriented pairs while enormous $(6726 \mathrm{kcal} / \mathrm{mol})$ for methyl $m$-bromocinnamate. The large increase in the lattice energy in the latter case is indicative of resistance from the cavity wall for the required reorganization. In terms of free volume within a reaction cavity the above conclusion could be interpreted as a pair of 7-methoxycoumarin 
molecules containing a greater amount of free volume than in the case of methyl $\mathrm{m}$ bromocinnamate thus the reaction cavities in 7-methoxycoumarin crystals can tolerate the large motions executed during dimerization. More important than availability of free volume is its presence in the right place.

\section{IVb. Role of cavity-guest interactions}

An important feature of reaction cavities in many organized media is that their functionalities can selectively interact with the solute molecules. The reaction cavity wall is termed "active" when there is an attractive or repulsive interaction between a guest molecule and the wall of the cavity and the interaction may serve as a template for the guest as it proceeds to products (Figure 22). The presence of active walls necessitates consideration of possible wall-guest interactions during the prediction (and rationalization) of product selectivity. If sufficiently attractive or repulsive, the interactions will influence the location or conformation of guest molecules and thereby the final products. Additionally, if the interactions are to have a discernable influence on the course of the transformations, they must persist for times at least comparable to those required for the reaction to occur. Interactions may vary from weak van der Waal's forces, to hydrogen bonds to strong electrostatic forces between charged centers. For example, a number of hosts capable of forming inclusion complexes (such as cyclodextrins, urea, cucurbuturil and deoxycholic acid) contain functional groups that can form hydrogen bonds with guest molecules; silanol groups of silica surfaces may orient the adsorbed molecules through hydrogen bonding; the large number of cations on the surfaces of clays and zeolites can interact electrostatically with guests. Most reaction cavity walls may, in fact, be "active" to some extent. Understanding this feature generally allows exploitation of the medium to the maximum extent.

Examples of asymmetric induction chosen from studies in zeolites illustrate the importance of cavity-guest interactions. Two sets of photoreactions illustrated in Schemes 23 and 24 demonstrate the uniqueness of cation-guest interactions in achieving chiral selectivity in photoreactions within zeolites. In one set of molecules (Scheme 23) the less than $10 \%$ diastereomeric excess (de) obtained in isotropic solution is enhanced within cation exchanged zeolites to over 80\%. In the other (Scheme 24), moderate 
enantiomeric excess (ee) is observed within zeolites from the lack of any chiral induction in solution. Examination of the results observed within zeolites in the case of (S(-)-1phenylethyl amide cis-diphenylcyclopropane carboxylic acid (Scheme 23) supports the conclusion that the cation-guest interaction is key to the observed chiral selectivity.[121] The de is dependent on the nature of the alkali metal ion (decreased with increasing mass; e.g., $\mathrm{NaY}$ to $\mathrm{CsY}$ ) and water content (decreased with higher water content of the NaY). The de is very low for silica gel, a surface lacking cations and decreased significantly on changing the $\mathrm{Si} / \mathrm{Al}$ ratio of $\mathrm{LiY}$ zeolite from 2.4 to 40 . Such a change in the $\mathrm{Si} / \mathrm{Al}$ ratio results in a drastic decrease in the number of cations per unit cell from 55 to 5 . For the current discussion important point to note is that interactions (cation-guest) that do not persist in isotropic solution do so in an organized media and play a critical role in controlling photoproduct selectivity.

\section{IVc. Role of interface}

A reaction cavity can be pictured as an enclosure that reduces the mobility of the reactant molecules and provides a boundary that the reactant molecules may not cross without overcoming an energy barrier. In certain organized media the boundary of the 'reaction cavity' may separate two phases of very different properties. As mentioned earlier the boundary of a micelle separates the non-polar hydrocarbon interior from the polar aqueous exterior. In Section IIIa we discussed the use of an interface to align molecules for photocycloaddition reactions. The example provided below illustrates how interface could be utilized to control molecular conformations and thereby photoproducts.

Photoproduct selectivity in unimolecular reactions can be achieved through conformational control of the reactant molecule at the interface. For example, the only observable Norrish type I reaction in organic solvents with benzoin alkyl ethers (Scheme 25) is completely suppressed and Norrish-Yang reaction becomes the major pathway for benzoin methyl ether in cetyl trimethyl ammonium bromide and cetyl trimethyl ammonium chloride micelles (Scheme 26) due to the cage effect as well as the conformational control at the interface. Of the two conformations A and B shown in Scheme 27 for benzoin methyl ether, conformer B at the micellar interface (probably from the preference for the polar carbonyl and methoxy groups to be situated in the 
aqueous phase) leads to the Norrish-Yang products. Production of only the parasubstituted benzophenone from benzoin octyl ether in the same medium is consistent with the rationale that the long alkyl chain would prefer to remain in the hydrocarbon interior to lead predominantly to conformer $\mathbf{A}$ at the micellar interface (Scheme 27). Such a condition would not favor Norrish-Yang reaction.

\section{Influence of the Supramolecular Assemblies on Excited State Processes: Exemplars}

In the above presentation we highlighted the features of supramolecular assemblies that could be utilized to modify excited state chemistry. We attempted to bring out common features of various types of supramolecular structures so that direct comparison between each of them could be made. For example one might ask how is cyclodextrin different from micelles, should I use zeolite or clay to bring about a desired change etc. More importantly, some of the features are unique to supramolecular assemblies and one can't achieve the same goal in isotropic solution. To highlight this aspect of supramolecular photochemistry we use two examples, room temperature phosphorescence (RTP) and chiral photochemistry.

\section{Va. Influence of Media on a Photophysical Process-Phosphorescence}

Phosphorescence from organic molecules is an area where organized media have made a tremendous impact. Phosphorescence emission from the triplet state of organic molecules is rare in neat solvents at room temperature. Intense phosphorescence has been observed at room temperature from arenes solubilized in sodium lauryl sulfate (SDS) micelles in the presence of $\mathrm{Tl}^{+}$or $\mathrm{Ag}^{+}$ions (Figure 23).[264, 265] Cyclodextrin has also proven to be an excellent matrix to observe phosphorescence at room temperature. In this case co-inclusion of a heavy atom inert co-guest is required to induce phosphorescence. For example, inclusion of aromatic molecules within cyclodextrin containing brominated alcohols (2-bromoethanol, 2,3-dibromo-1-propanol) results in RTP of aromatic molecules.[266-270]

Phosphorescence at room temperature (RTP) from a number of arenes and olefins has been observed with heavy-cation exchanged zeolites media. For example, 
naphthalene, when included in the X-type faujasites $\mathrm{M}^{+} \mathrm{X}^{-}$exchanged with $\mathrm{Rb}^{+}$or $\mathrm{Cs}^{+}$, exhibits intense RTP along with weak fluorescence. In faujasites exchanged with $\mathrm{Li}^{+}$, $\mathrm{Na}^{+}$, or $\mathrm{K}^{+}$ions, intense fluorescence and weak phosphorescence have been observed (Figure 24). [271, 272] The correlation of phosphorescence intensity with cation mass clearly suggests an external heavy-atom perturbation. The magnitude of the heavy-atom effect observed in zeolites is significantly larger than that observed for a similar cation included 1,5-naphtho-22-crown-6 complexes, where the cation is rigidly held over the naphthalene $\pi$ face. The observed heavy atom effect with zeolite samples is nearly as large as for a series of 1-halonaphthalenes where the perturbers are covalently attached to the chromophore. The unusually high external heavy-atom effect observed here is attributed to the close approach between naphthalene and the heavy cation, that is reenforced by the zeolite supercage, and to the presence of more than one heavy cation per supercage, which leads to high effective concentrations of the heavy atom in the vicinity of the naphthalene.

The normally absent or weak phosphorescence from olefins (e.g., trans-stilbene, 1,6-all-trans-diphenylhexatriene) even in the presence of conventional heavy atom solvents at low temperature is observable at room temperature due to the efficient close proximity between the cations and the olefin within a zeolite (Figure 25).[271]

Before closing this section we like to point out that RTP has been observed on silica surfaces and papers impregnated with heavy metal salts such as thalium nitrate.[273-276] Thus the heavy cation and its proximity to the emitter are the key factors that lead to enhancement of phosphorescence and the confined container prevents quenching by oxygen and by itself (self-quenching; see below).

The final example in this category deals with phosphorescence of thioketones, molecules analogous to ketones.[277] These molecules with high values of $\mathrm{k}_{\mathrm{ST}}$ and $\mathrm{k}_{\mathrm{P}}$ do not phosphoresce in solution at room temperature due to quenching by oxygen and by ground state thioketone molecules (self-quenching) at diffusion controlled rates. Supramolecular approach helps solve the lack of measurable phosphorescence of these thioketones at concentration above $10^{-6} \mathrm{M}$. For example, thiocamphor (Figure 26) showing no phosphorescence in aqueous solution $\left(10^{-4} \mathrm{M}\right)$, phosphoresces intensely when encapsulated in a 2:2 complex with octa acid in water. The absence of self-quenching 
though the effective concentration within the complex is $0.35 \mathrm{M}$ is ascribed to the supramolecular pre-organization of the two incarcerated thioketones into a relative orientation such that the two $\mathrm{C}=\mathrm{S}$ groups are situated at a significant distance from each other. Thus self-quenching is eliminated by host enforced pre-organization occurring as a result of guest-host complexation. Furthermore, the thioketone is also protected from quenching by oxygen by the octa acid capsule.

\section{Vb. Asymmetric photochemical transformations within zeolites}

Following the communication on the use of optically active sensitizers in the photosensitized isomerization of cis-diphenylcyclopropane by Hammond and Cole in 1965 [278] several groups have performed enantio- and diastereoselective phototransformations, both in solution and the solid state.[279-281] The best results in solution have been obtained via the chiral auxiliary methodology, yielding in select examples diastereomeric excesses (de) close to 100\%.[282, 283] There are relatively few examples of asymmetric induction during photolysis of achiral molecules in the crystalline state [70] due to the limited probability of the crystallization of achiral molecules crystallizing in chiral space groups. [284] Scheffer and co-workers have provided a number of examples that yield photoproducts in very high ee (or de) in the crystalline state using the "ionic chiral auxiliary approach" that facilitates the crystallization of achiral molecules in chiral space groups.[285-290]. Recognizing the above stated problem of crystallizing achiral molecules in chiral space groups, several researchers have explored chiral hosts as the reaction medium starting from Natta on the photopolymerization of 1,3-dienes included in the channels of optically active perhydrotriphenylene,[291] to the most achieved success by Toda using chiral diol hosts.[292, 293] While crystalline and host-guest assemblies have been extremely useful in conducting enantioselective photoreactions, their general applicability has been limited. Not all molecules crystallize alone or in the presence of organic host systems. Due to the paucity of reliable prediction capabilities of the molecular packing that control the reactivity of molecules in the crystalline state and in solid host-guest assemblies successful use of above methods offers no guarantee that the products will react in the crystalline state. Hence readily available and inexpensive zeolites have been employed 
as media to bring about asymmetric induction in photochemical reactions. In this section the different approaches to bring about chiral induction during photoreaction within zeolites will be discussed. Discussing the various methods utilized to achieve chiral induction in photochemical reactions is beyond the scope of the current review. The value of supramolecular assemblies in chiral photochemistry will be highlighted with examples from zeolite and cyclodextrins.

Provision of the asymmetric environment within a zeolite by adsorption of the chiral inductor such as ephedrine ('chiral inductor method') renders the nonchiral interior surface of the zeolite "locally chiral". This simple method affords easy isolation of the chiral photoproduct. The chiral inductor used will determine the magnitude of the enantioselectivity of the photoproduct.

Employing chirally modified zeolites as reaction media requires inclusion of two different molecules, a chiral inductor $(\mathrm{CI})$ and a reactant $(\mathrm{R})$, within the interior space of the zeolite, which by its very nature does not allow quantitative asymmetric induction. The six possible occupancies of molecules CI and R included within a given zeolite are shown in Figure 27: cages containing a single R or CI molecule (type C, Type E), two R or CI molecules (type A, type D), an R and one CI (type B) and neither R or CI molecule (type F). Of the three possible scenarios of photoreaction of $\mathrm{R}$, namely $\mathrm{A}, \mathrm{B}$ and $\mathrm{C}$, depending on the interaction between the chiral inductor and the reactant asymmetric induction is possible from B alone. High asymmetric induction therefore requires the placement of each reactant molecule next to a chiral inductor i.e., enhancement of the ratio of type $\mathrm{B}$ cages to the sum of types $\mathrm{A}$ and $\mathrm{C}$.

Reactions such as viz., electrocyclic reactions,[294-296] the Zimmerman di$\pi-$ methane reaction,[297] the oxa-di- $\pi$-methane rearrangement,[298] Yang cyclization,[299, 300] geometric isomerization of 1,2-diphenylcyclopropane derivatives, [121, 122, 301-307] and the Schenk-ene reaction[297] that gave racemic products even in the presence of chiral inductors in solution (see Schemes 28 and 29 for selected examples) were explored to examine the viability of this approach. Two reactions gave respectable ee's within $\mathrm{NaY}$, namely, the photocyclization of tropolone phenylethyl ether (78\%, Scheme 24)[294-296] and photocyclization of 1-(3phenylpropyl)-2-pyridone (50\%, Scheme 24) while the others gave moderate ee's 
(15-50\%).[308] The results within zeolites are significant and mechanistically interesting relative to the racemic products in solution. Best results were obtained with ephedrine, norephedrine or pseudoephedrine as the chiral inductor.[121]

High stereoselectivity is unlikely by the above method without the development of a strategy placing each reactant molecule next to a chiral inductor within a zeolite. This led to the chiral auxiliary method in which the chiral perturber was covalently bound to the reactant. [285, 286, 295, 301, 305, 306, 309, 310] In this approach most cages are expected to contain both the reactant as well as the chiral inductor components (Figure 27 II). The diastereomeric excesses (de) obtained within zeolites using the chiral auxiliary method explored in several systems (for selected examples see Scheme 29) were far superior to those in solution. Diastereomeric excesses of $>50 \%$ have routinely been obtained within MY zeolites for systems that yield photoproducts in 1:1 diastereomeric ratio in solution.

Despite high stereoselection during various phototransformations with the chiral auxiliary method, residence of the reactant and its covalently linked chiral inductor in different cages by adopting an extended conformation that could result in $<100 \%$ de (type B in Figure 27II) is possible. To provide an asymmetric environment to such molecules chirally modified Y zeolites were employed as the reaction media (Figure 27 III). Within $(-)$-ephedrine modified $\mathrm{NaY}$, the de in the photoproduct from tropolone-2-methylbutyl ether increased from 53\% to 90\% (Figure 28),[285].

In addition to zeolites, inherently chiral cyclodextrins thus eliminating the need for additional chiral inductors for photochemical reactions that have been explored as media both in solution and solid state have produced low to moderate chiral induction. [311-325] A few examples are listed below in Schemes 30-33.

\section{Perspectives}

Supramolecular photochemistry has its origin in organic solid state photochemistry that has been explored since late 1800.[326] The explosion of the interest in this field during the last forty years is evident from the more than 200 reviews and book chapters in the literature. During this time, from the perspective of photochemists the term supramolecular has included micelles, LB films, surfaces of silica, clay and zeolites, liquid crystals, organic and inorganic host-guest complexes in 
solution and solid state and biological assemblies such as proteins, membranes and proteins. Duplicating enzyme chemistry has been the hidden but unaccomplished goal in most cases. Though realized in most examples, selectivity in products comparable to the enzymes in biological systems has been elusive. Making reactions catalytic has been a major challenge. These studies have nevertheless advanced the knowledge on weak interactions and behavior of molecules in confined spaces. The challenge of making these selective reactions catalytic and identifying the most suitable supramolecular assembly for a given goal[180, 218] lies in understanding the dynamics of supramolecular assemblies, especially in aqueous solution.[327-329] The concepts of supramolecular photochemistry have recently found applications in the capture, storage and use of sunlight.[330-333] Realization of the full potential of this technique awaits better understanding of the behavior of supramolecular assemblies on active and inactive solid surfaces.[334-336]

\section{Acknowledgement}

VR is grateful to the National Science Foundation for financial support. A part of this review was written while VR was at the Indian Institute of Science, Bangalore as Fulbright-Nehru Distinguished Chair and at Tokyo Metropolitan University, Tokyo as JSPS Invitational Fellow.

\section{References}

[1] J.-M. Lehn, Supramolecular Chemistry: Concepts and perspectives, VCH, New York, 1995.

[2] J.K. Thomas, Effect of structure and Charge on Radiation-Induced Reactions in micellar systems, Acc. Chem. Res., 10 (1977) 133-138.

[3] D.G. Whitten, Photochemical reactions of surfactant molecules in condensed monolayer asemblies-environmental control and modification of reactivity., Angew. Chem. Int. Ed. Engl., 18 (1979) 440-450. 
[4] N.J. Turro, M. Gratzel, A.M. Braun, Photophysical and photochemical processes in micellar systems, Angew. Chem. Int. Ed. Engl., 19 (1980) 675-696.

[5] D.G. Whitten, J.C. Russell, R.H. Schmell, Photochemical reactions in organised assemblies: Environmental effects on reactions occuring in micelles, vesicles, films and multilayer assemblies and at interfaces, Tetrahedron, 18 (1982) 24552487.

[6] V. Ramamurthy, Organic Photochemistry in Organized Media, Tetrahedron, 42 (1986) 5753-5839.

[7] R.G. Weiss, Thermotropic liquid crystals as reaction media for mechanistic investigations, Tetrahedron, 44 (1988) 3413-3475.

[8] P. de Mayo, Superficial Photochemistry, Pure Appl. Chem., 54 (1982) 16231632.

[9] N.J. Turro, Photochemistry of organic molecules in microscopic reactors, Pure Appl. Chem., 58 (1986) 1219-1228.

[10] K. Kalyanasundaram, Photochemistry in Microheterogeneous Systems, Academic Press, Inc., New York, 1987.

[11] V. Ramamurthy, Photochemistry in Organized and Constrained Media, in: V. Ramamurthy (Ed.), Wiley-VCH, New York, 1991.

[12] H.-J. Schneider, H. Durr, Frontiers in Supramolecular Organic Chemistry and Photochemistry, in, VCH, New York, 1991, pp. 485.

[13] V. Balzani, F. Scandola, Supramolecular Photochemistry, Ellis Horwood Limited, Chichester, 1991.

[14] A. Natarajan, B. Bhogala, Biomolecular photoreactions in the crystalline state, in: V. Ramamurthy, Y. Inoue (Eds.) Supramolecular Photochemistry, John Wiley\& Sons, Inc., 2011, pp. 175-228.

[15] A. Natarajan, V. Ramamurthy, Solvent-free photosynthesis of cyclobutanes: Photodimerization of crystalline olefins, in: Z. Rappoport, J.F. Liebman (Eds.) The Chemistry of Cyclobutanes, John Wiley \& Sons, Ltd, Chichester, 2005, pp. 807-872.

[16] V. Ramamurthy, K. Venkatesan, Photochemical reactions of organic crystals, Chem. Rev., 87 (1987) 433-481. 
[17] G.M.J. Schmidt, The photochemistry of the solid state, in: Reactivity of the photoexcited organic molecule, Interscience, New York, 1967, pp. 227-288.

[18] G.M.J. Schmidt, Photodimerization in the solid state, Pure Appl. Chem., 27 (1971) 647-678.

[19] M.D. Cohen, Structural factors in some solid state photodimerizations, Mol. Cryst. Liq. Cryst., 9 (1969) 287-295.

[20] M.D. Cohen, The photochemistry of organic solids, Angew. Chem. Int. Ed. Engl., 14 (1975) 386-393.

[21] M.D. Cohen, Recent Research in Topochemistry at the Weizmann Institute of Science, in: D. Ginsburg (Ed.) G. M. J. Schmidt et al. Solid State Photochemistry, Verlag Chemie, GmbH, Weinheim, 1976, pp. 233-254.

[22] M.D. Cohen, Excimers in crystals, Mol. Cryst. Liq. Cryst, 50 (1979) 1-10.

[23] M.D. Cohen, Solid-state photochemical reactions, Tetrahedron, 43 (1987) 12111224.

[24] L. Addadi, M. Cohen, M. Lahav, L. Leiserowitz, Solid-state organic chemistry Scope and trends, J. Chim. Phys., 83 (1986). page no

[25] L. Addadi, M. Lahav, Towards the planning and execution of an "absolute" asymmetric synthesis of chiral dimers and polymers with quantitative enantiomeric Yield., Pure Appl. Chem., 51 (1979) 1269-1284.

[26] B.S. Green, R. Arad-Yellin, M.D. Cohen, Stereochemistry and organic solidstate reactions, in: Top. Stereochem., 1986, pp. 131-218.

[27] B.S. Green, M. Lahav, D. Rabinovich, Assymetric synthesis via reactions in chiral crystals, Acc. Chem. Res., 12 (1979) 191-197.

[28] J.R. Scheffer, The solid-state ionic chiral auxiliary approach to asymmetric induction in photochemical reactions, in: Y. Inoue, V. Ramamurthy (Eds.) Molecular and Supramolecular Photochemistry, Marcel Dekker, 2004, pp. 463483.

[29] J.R. Scheffer, Crystal lattice control of unimolecular photorearrangements, Acc. Chem. Res., 13 (1980) 283-290.

[30] J.R. Scheffer, Geometric requirements for intramolecular photochemical hydrogen atom abstraction: studies based on a combination of solid state 
chemistry and X-ray crystallography, in: G.R. Desiraju (Ed.) Organic Solid State Chemistry, Elsevier Science Publishers B. V., 1987, pp. 1-45.

[31] J.R. Scheffer, In the footsteps of Pasteur: asymmetric induction in the photochemistry of crystalline ammonium carboxylate salts, Can. J. Chem., 79 (2001) 349-357.

[32] J.R. Scheffer, M. Garcia-Garibay, O. Nalamasu, The influence of the molecular crystalline environment on organic photorearrangements, in: A. Padwa (Ed.) Organic Photochemistry, Marcell Decker, Inc., New York, 1987, pp. 249-347.

[33] J.R. Scheffer, P.R. Pokkuluri, Unimolecular Photoreactions of organic crystals: the medium is the message, in: V. Ramamurthy (Ed.) Photochemistry in organized and constrained media, VCH Publishers, Inc., 1991, pp. 185-246.

[34] J.R. Scheffer, W. Xia, Asymmetric induction in organic photochemistry via the solid-state ionic chiral auxiliary approach, Top. Curr. Chem., 254 (2005) 233262.

[35] J.N. Gamlin, R. Jones, M. Leibovitch, B. Patrick, J.R. Scheffer, J. Trotter, The ionic auxiliary concept in solid state organic photochemistry, in: Acc. Chem. Res., 1996, pp. 203-209.

[36] H. Ihmels, J.R. Scheffer, The Norrish type II reaction in the crystalline state: toward a better understanding of the geometric requirements for gammahydrogen atom abstraction, Tetrahedron, 55 (1999) 885-907.

[37] L. Caswell, M.A. Garcia-Garibay, J.R. Scheffer, J. Trotter, Optical activity can be created from "Nothing". J. Chem. Edu., 70 (1993) 785-787.

[38] M. Leibovitch, G. Olovosson, J.R. Scheffer, J. Trotter, Absolute configuration correlation studies in solid state organic photochemistry, Pure Appl. Chem., 69 (1997) 815-823.

[39] H.E. Zimmerman, Five decades of mechanistic and exploratory organic photochemistry, Pure Appl. Chem., 78 (2006) 2193-2203.

[40] H.E. Zimmerman, E.E. Nesterov, Development of experimenntal and theoretical crystal lattice organic photochemistry: The quantitative cavity. Mechanistic and exploratory organic photochemistry., Acc. Chem. Res., 35 (2002) 77. 
[41] M.A. Garcia-Garibay, Chemical reactivity in organized media: statistical entropy and information in crystals and enzymes, Curr. Opin. Solid St. M , 3 (1998) 399406.

[42] M.A. Garcia-Garibay, Engineering Carbene Rearrangements in Crystals: From Molecular Information to Solid-State Reactivity, Acc. Chem. Res., 36 (2003) 491-498.

[43] M.A. Garcia-Garibay, Molecular Crystals on the Move: From Single-Crystal-toSingle-Crystal Photoreactions to Molecular Machinery, Angew. Chem. Int. Ed. , 46 (2007) 8945-8947.

[44] M.A. Garcia-Garibay, The entropic enlightenment of organic photochemistry: strategic modifications of intrinsic decay pathways using an information-based approach, Photochem. Photobiol. Sci., 9 (2010) 1574-1588.

[45] M.A. Garcia-Garibay, A.E. Constable, J. Jernelius, T. Choi, D. Cimeciyan, S.H. Shin, Diffraction, spectral and thermal analysis of mixed crystalline phases. Mechanisms of solid-to-solid organic reactions, in: L. Echegoyen, A.E. Kaifer (Eds.) Physical supramolecular chemistry, 1996, pp. 485-508.

[46] A.E. Keating, M.A. Garcia-Garibay, Photochemical solid-to-solid reactions, in: V. Ramamurthy, K.S. Schanze (Eds.) Molecular and supramolecular Photochemistry, Marcel Dekker, Inc.,, New York, 1998, pp. 195-248.

[47] C.J. Mortko, M.A. Garcia-Garibay, Engineering Stereospecific Reactions in Crystals: Synthesis of Compounds with Adjacent Stereogenic Quaternary Centers by Photodecarbonylation of Crystalline Ketones, in: S.E. Denmark, J.S. Siegel (Eds.) Topics in Stereochemistry, John Wiley \& Sons, Inc., 2006, pp. 205253.

[48] L.M. Campos, M.A. Garcia-Garibay, Reactive Intermediates in Crystals: Form and Function, in: M.S. Platz, R.A. Moss, M.J. Jones (Eds.) Reviews of Reactive Intermediate Chemistry, John Wiley \& Sons, Inc., New Jersey, 2007, pp. 271331.

[49] D.-K. Bucar, G.S. Papaefstathiou, T.D. Hamilton, Q.L. Chu, I.G. Georgiev, L.R. MacGillivray, Template-controlled reactivity in the organic solid state by 
principles of coordination-driven self-assembly, Eur. J. Inorg. Chem., (2007) 4559-4568.

[50] E. Elacqua, T. Friscic, L. MacGillivray, [2.2]Paracyclophane as a target of the organic solid state: Emergent properties via supramolecula construction, Israel J. Chem., 52 (2012) 53-59.

[51] T. Friscic, T.D. Hamilton, G.S. Papaefstathiou, L.R. MacGillivray, A templatecontrolled solid-state reaction for the organic chemistry laboratory., J. Chem. Edu., 82 (2005) 1679-1681.

[52] T. Friscic, L.R. Macgillivray, Cyclophanes and ladderanes: molecular targets for supramolecular chemists. , Supramol. Chem., 17 (2005) 47-51.

[53] T. Friscic, L.R. MacGillivray, Template-controlled solid-state synthesis: Toward a general form of covalent capture in molecular solids, in: E.R.T. Tiekink, J.J. Vittal (Eds.) Frontiers in Crystal Engineering, John Wiley \& Sons, Ltd., 2006, pp. 51-66.

[54] I.G. Georgiev, L.R. MacGillivray, Metal mediated reactivity in the organic solid state: from self-assembled complexes to metal-organic frameworks, Chem. Soc. Rev., 36 (2007) 1239-1248.

[55] L.R. MacGillivray, Organic synthesis in the solid state via hydrogen-bond-driven self-assembly. , J. Org. Chem., 73 (2008) 3311-3317.

[56] L.R. MacGillivray, G.S. Papaefstathiou, T. Friscic, T.D. Hamilton, D.-K. Bucar, Q. Chu, D.B. Varshney, I.G. Georgiev, Supramolecular control of reactivity in the solid state: from templates to ladderanes to metal-organic frameworks, Acc. Chem. Res., 41 (2008) 280-291.

[57] K. Tanaka, F. Toda, Solvent-free organic synthesis, Chem. Rev., 100 (2000) 1024-1074.

[58] F. Toda, Reaction control of guest compounds in host-guest incluson complexes, Top. Curr. Chem., 149 (1988) 211-238.

[59] F. Toda, Enantiocontrol of photoreactions in the solid state, Mol. Cryst. Liq. Cryst., 161 (1988) 355-362.

[60] F. Toda, Reaction control by a host-guest complexation method, J. Inclusion Phenom. Mol. Recog. Chem., 7 (1989) 247-256. 
[61] F. Toda, Selective reactions in the solid state and organic solid-solid reactions, Mol. Cryst. Liq. Cryst., 187 (1990) 41-48.

[62] F. Toda, Solid state organic chemistry: efficient reactions, remarkable yields, and stereoselectivity, Acc. Chem. Res., 28 (1995) 480-486.

[63] F. Toda, Crystalline inclusion complexes as media of molecular recognitions and selective reactions, Pure Appl. Chem., 73 (2001) 1137-1145.

[64] F. Toda, Thermal and photochemical reactions in the solid state, in: Top. Curr. Chem., 2005, pp. 1-40.

[65] F. Toda, K. Tanaka, H. Miyamoto, Enantioselective photoreactions in the solid state, in: V. Ramamurthy, K.S. Schanze (Eds.) Understanding and manipulating excited-state processes, Marcel Dekker, New York, 2001, pp. 385-425.

[66] K. Tanaka, F. Toda, Organic photoreactions in the solid state, in: F. Toda (Ed.) Organic solid state reactions, Kluwer, New York, 2002, pp. 109-157.

[67] Y. Ito, Photoreactivity of carbonyl compounds in the solid state, in: M. Anpo, T. Matsuura (Eds.) Photochemistry on solid surfaces, Elsevier, Amsterdam, 1989, pp. 469-480.

[68] Y. Ito, Solid-state photoreactions in two-component crystals, Synthesis, (1998) $1-32$.

[69] Y. Ito, Solid-state organic photochemistry of mixed molecular crystals, in: V. Ramamurthy, K. Schanze (Eds.) Molecular and Supramolecular Photochemistry, Marcell Dekker, New York, 1999, pp. 1-70.

[70] M. Sakamoto, Absolute asymmetric synthesis from achiral molecules in the chiral crystalline environment., Chem. Eur. J., 3 (1997) 684-689.

[71] M. Sakamoto, Absolute asymmetric photochemistry using spontaneous chiral crystallization, in: Y. Inoue, V. Ramamurthy (Eds.) Chiral Photochemistry, Marcel Dekker, Inc.,, New York, 2004, pp. 415-461.

[72] M. Sakamoto, Photochemical Aspects of thiocarbonyl compounds in the solidstate, Top. Curr. Chem., 254 (2005) 207-232.

[73] M. Sakamoto, Spontaneous chiral crystallization of achiral materials and absolute asymmetric photochemical transformation using the chiral crystalline environment, J. Photoch. Photobio. C, 7 (2006) 183-196. 
[74] M. Irie, Photochromic diarylethenes for photonic devices, Pure Appl. Chem., 68 (1996) 1367-1371.

[75] M. Irie, Photochromism of diarylethenes in confined reaction spaces, in: V. Ramamurthy, K.S. Schanze (Eds.) Molecular and Supramolecular Photochemistry, Marcel Dekker, Inc., New york, 2000, pp. 111-141.

[76] M. Irie, Diarylethenes for memories and switches, Chem. Rev., 100 (2000) 16851716.

[77] M. Irie, Photochromism of diarylethene single crystal and single molecules, Mol. Cryst. Liq. Cryst., 430 (2005) 1-7.

[78] M. Irie, Photochromism and molecular mechanical devices, Bull. Chem. Soc. Jpn., 81 (2008) 917-926.

[79] M. Morimoto, M. Irie, Photochromism of diarlethene single crystal: crystal structure and photochromic performance, Chem. Commun., (2005) 3895-3905.

[80] M. Morimoto, M. Irie, Photochromism of multicomponent diarylethene crystals, in: V. Ramamurthy, Y. Inoue (Eds.) Supramolecular Photochemistry, John Wiley\& Sons, Inc., 2011, pp. 267-284.

[81] P. Coppens, What can time-resolved diffraction tell us about transient species?: excited-state structure determination at atomic resolution, Chem. Commun., (2003) 1317-1320.

[82] P. Coppens, S.-L. Zheng, Real-time crystallography of photoinduced processes in supramolecular framework solids, in: V. Ramamurthy, Y. Inoue (Eds.) Supramolecular Photochemistry, John Wiley\& Sons, Inc., 2011, pp. 155-174.

[83] K. Amimoto, T. Kawato, Photochromism of organic compounds in the crystal state, J. Photoch. Photobio. C, 6 (2005) 207-226.

[84] D. Braga, F. Grepioni, Reactions between or within molecular crystals, Angew. Chem. Int. Ed., 43 (2004) 4002-4011.

[85] D. Braga, F. Grepioni, Making crystals from crystals: a green route to crystal engineering and polymorphism, Chem. Commun., (2005) 3635-3645.

[86] L.X. Chen, Taking snapshots of photoexcited molecules in disordered media by using pulsed synchrotron X-rays, Angew. Chem. Int. Ed., 43 (2004) 2886-2905. 
[87] E. Hadjoudis, I.M. Mavridi, Photochromism and thermochromism of Schiff bases in the solid state: structural aspects, Chem. Soc. Rev., 33 (2004) 579-588.

[88] M.D. Hollingsworth, J.M. Mcbride, Photochemical mechanism in single crystals: FTIR studies of diacyl peroxides, in: Advances in Photochemistry, 1990, pp. 279-379.

[89] M. Kaftory, Structural aspects and templation of photochemistry in solid-state supramolecular systems, in: V. Ramamurthy, Y. Inoue (Eds.) Supramolecular Photochemistry, John Wiley\& Sons, Inc., 2011, pp. 229-266.

[90] S.K. Kearsley, The Prediction of chemical reactivity within organic crystals using geometric criteria, in: G.R. Desiraju (Ed.) Organic Solid State Chemistry, Elsevier Science Publishers B. V., 1987, pp. 69-115.

[91] G.K. Kole, J.J. Vittal, Solid-state reactivity and structural transformations involving coordination polymers, Chem. Soc. Rev., 42 (2013) 1755-1775.

[92] H. Koshima, Solid-state bimolecular photoreactions in two-component molecular crystals, in: F. Toda (Ed.) Organic Solid-State reactions, Kluwer Academic Publishers, 2002, pp. 189-269.

[93] H. Koshima, Chiral solid-state photochemistry including supramolecular approaches, in: Y. Inoue, V. Ramamurthy (Eds.) Chiral Photochemistry, Marcel Dekker, Inc.,, New York, 2004, pp. 485-531.

[94] J.M. McBride, The Role of local stress in solid state radical reactions, Acc. Chem. Res., 16 (1983) 304-312.

[95] J.M. McBride, B.E. Segmuller, M.D. Hollingsworth, D.E. Mills, B.A. Weber, Mechanical stress and reactivity in organic solids, Science, 234 (1986) 830-835.

[96] M. Nagarathinam, A.M.P. Peedikakkal, J.J. Vittal, Stacking of double bonds for photochemical [2+2] cycloaddition reactions in the solid state, Chem. Commun., (2008) 5277-5288.

[97] M. Nagarathinam, J.J. Vittal, A rational approach to crosslinking of coordination polymers using the photochemical [2+2] cycloaddition reaction., Macromol. Rapid Commun., 27 (2006) 1091-1099.

[98] V.E. Shklover, T.V. Timofeeva, Reactions in organic crystals, Russ. Chem. Rev., 54 (1985) 619-644. 
[99] Y. Sonada, Solid-state [2+2] photodimerization and photopolymerization of a,wdiarylpolyene monomers: effective utilization of noncovalent intermolecular interactions in crystals, molecules, 16 (2011) 119-148.

[100] Y. Sonoda, [2+2]Photocycloadditions in the solid state, in: W. Horspool, F. Lenci (Eds.) CRC Handbook of Organic Photochemistry and Photobiology, CRC Press Boca Raton, 2004, pp. 73-71 - 73-15.

[101] C.R. Theocharis, Dimerization and polymerization of enones in the fluid and solid states, in: S. Patai, Z. Rappoport (Eds.) The Chemistry of Enones, John Wiley \& Sons, New York, 1989, pp. 1133-1176.

[102] C.R. Theocharis, W. Jones, Topotactic and topochemical photodimerization of benzylidenecyclopentanones, in: G.R. Desiraju (Ed.) Organic Solid State Chemistry, Elsevier Science Publishers B. V., 1987, pp. 47-68.

[103] J.M. Thomas, Organic reactions inthe solid state: accident and design, Pure Appl. Chem., 51 (1979) 1065-1082.

[104] J.M. Thomas, S.E. Morsi, J.P. Desvergne, Topochemical phenomena in organic solid-state chemistry, in: V. Gold, H. Bethell (Eds.) Advances in Physical Organic Chemistry, Academic press., London, 1977, pp. 63-151.

[105] J.M. Thomas, Crystal engineering: origins, early adventures and some current trends, Cryst. Eng. Comm, 13 (2011) 4304-4306.

[106] I. Turowska-Tyrk, Structural transformations in organic crystals during photochemical reactions, J. Phys. Org. Chem., 17 (2004) 837-847.

[107] K. Venkatesan, Some aspects of bimolecular photoreactions in crystals, in: V. Boldyrev (Ed.) Reactivity of Molecular Solids, John Wiley \& Sons Ltd, 1999, pp. 89-131.

[108] K. Venkatesan, V. Ramamurthy, Biomolecular photoreactions in crystals, in: V. Ramamurthy (Ed.) Photochemistry in organized and constrained media, $\mathrm{VCH}$ Publishers, Inc., 1991, pp. 133-184.

[109] K. Vishnumurthy, T.N.G. Row, K. Venkatesan, Observations on the photochemical behavior of coumarins and related systems in the crystalline state, in: V. Ramamurthy, K.S. Schanze (Eds.) Molecular and Supramolecular Photochemistry, 2001, pp. 427-460. 
[110] J.J. Vittal, Supramolecular structural transformations involving coordination polymers in the solid state., Coord. Chem. Rev., 251 (2007) 1781-1795.

[111] R.G. Weiss, Photochemical processes in liquid crystals, in: V. Ramamurthy (Ed.) Photochemistry in organized and constrained media, VCH Publishers, Inc., 1991, pp. 603-690.

[112] R.G. Weiss, R.L. Treanor, A. Nunez, Norrish II reactions of rod-like ketones in ordered media comprised of rod-like layered solvent molecules., Pure Appl. Chem., 60 (1988) 999-1008.

[113] W. Gu, R.G. Weiss, Extracting fundamental photochemical and photophysical information from photorearrangements of aryl phenylacylates and aryl benzyl ethers in media comprised of polyolefinic films, J. Photoch. Photobio. C, 2 (2001) 117-137.

[114] S. Abraham, R.G. Weiss, Photochemical and photophysical studies of and in bulk polymers, in: V. Ramamurthy, Y. Inoue (Eds.) Supramolecular Photochemistry, John Wiley\& Sons, Inc., 2011, pp. 443-516.

[115] S.P. Spooner, D.G. Whitten, Photoreactions in monolayer films and langmuirblodgett assemblies, in: V. Ramamurthy (Ed.) Photochemistry in organized and constrained media, VCH Publishers, Inc., 1991, pp. 691-738.

[116] D.G. Whitten, D.W. Eaker, B.E. Horsey, R.H. Schmehl, P.R. Worsham, Photochemical and thermal reactions of porphyrins and organic surfactants in monolayer assemblies. Modification of reactivity in condensed hydrophobic microenvironments (I), Berich. Bunsen. Gesell, 82 (1978) 858-867.

[117] D.G. Whitten, F.R. Hopf, F.H. Quina, G. Sprintschnik, H.W. Sprintschnik, Photochemistry of organic chromophores incorporated into fatty acid monolayers, Pure Appl. chem., 49 (1977) 379-388.

[118] A. Joy, V. Ramamurthy, Chiral photochemistry within zeolites, Chem. Eur. J., 6 (2000) 1287-1293.

[119] V. Ramamurthy, Excited state chemistry of organic molecules included within zeolites, in: M. Anpo (Ed.) Surface Photochemistry John Wiley \& Sons, 1996, pp. 65-115. 
[120] V. Ramamurthy, Controlling photochemical reactions via confinement: zeolites, J. Photoch. Photobio. C, 1 (2000) 145-166.

[121] J. Sivaguru, A. Natarajan, L.S. Kaanumalle, J. Shailaja, S. Uppili, A. Joy, V. Ramamurthy, Asymmetric photoreactions within zeolites: role of confinement and alkali metal ions, Accounts Chem. Res., 36 (2003) 509-521.

[122] J. Sivaguru, J. Shailaja, S. Uppili, K. Ponchot, A. Joy, N. Arunkumar, V. Ramamurthy, Achieving enantio and diastereoselectivities in photoreactions through the use of a confined space, in: F. Toda (Ed.) Organic Solid-State reactions, Kluwer Academic Publishers, 2002, pp. 159-188.

[123] N.J. Turro, From boiling stones to smart crystals: supramolecular and magnetic isotope control of radical-radical reactions inside zeolites., Accounts Chem. Res., 33 (2000) 637-646.

[124] N.J. Turro, From molecular chemistry to supramolecular chemistry to superdupermolecular chemistry. Controlling covalent bond formation through non-covalent and magnetic interactions, Chem. Commun., (2002) 2279-2292.

[125] A. Corma, H. Garcia, Zeolite-based photocatalysts, Chem. Commun., (2004) 1443-1459.

[126] H. Garcia, H.D. Roth, Generation and reactions of organic radical cations in zeolites, Chem. Rev., 102 (2002) 3947-4007.

[127] J.C. Scaiano, H. Garcia, Intrazeolite Photochemistry: toward supramolecular control of molecular photochemistry, Accounts Chem. Res., 32 (1999) 783-793.

[128] G. Calzaferri, A. Devaux, Manipulation of energy transfer processes within the channels of L-zeolite, in: V. Ramamurthy, Y. Inoue (Eds.) Supramolecular Photochemistry, John Wiley\& Sons, Inc., 2011, pp. 285-388.

[129] G. Calzaferri, S. Huber, H. Maas, C. Minkowski, Host-guest antenna materials, Angew. Chem. Int. Ed., 42 (2003) 3732-3758.

[130] G. Calzaferri, H. Maas, M. Pauchard, M. Pfenniger, S. Megelski, A. Devaux, Supramolecularly Organized luminescent dye molecules in the channels of zeolite L, in: D.C. Neckers, G.V. Bunau, W.S. Jenks (Eds.) Advances in Photochemistry, John Wiley \& Sons, Inc., 2002, pp. 1-50. 
[131] P.K. Dutta, S. Chakraborty, Lightactivated processes with zeolites: recent developments, in: S.L. Suib (Ed.) New and Future Developments in Catalysis, Elsevier, Amsterdam, 2013, pp. 49-62.

[132] P.K. Dutta, M. Ledney, Charge-transfer processes in zeolites: toward better artificial photosynthetic models, in: G.J. Meyer (Ed.) Progress in Inorganic Chemistry, John Wiley \& Sons, Inc., 1997, pp. 209-271.

[133] M.N. Chretien, Supramolecular Photochemistry in Zeolites: From catalysts to sunscreens, Pure Appl. Chem., 79 (2007) 1-20.

[134] E.L. Clennan, Molecular Oxygenations in Zeolites, in: V. Ramamurthy, K.S. Schanze (Eds.) Molecular and Supramolecular Photochemistry, Marcel Dekker, 2003, pp. 275-308.

[135] E.L. Clennan, Mechanisms of oxygenations in zeolites, in: Advances in Physical Organic Chemistry, Elsevier Ltd, 2008, pp. 225-269.

[136] H. Frei, F. Blatter, H. Sun, Photocatalyzed oxidation of hydrocarbons in zeolite cages, Chemtech, 26 (1996) 24-30.

[137] S. Vasenkov, H. Frei, Photo-Oxidation in Zeolites, in: V. Ramamurthy, K.S. Schanze (Eds.) Molecular and Supramolecular Photochemistry, Marcel Dekker, 2000, pp. 295-323.

[138] K.B. Yoon, Electron- and charge- transfer reactions within zeolites, Chem. Rev., 93 (1993) 321-339.

[139] K.B. Yoon, Charge and electron transfer reactions in zeolites, in: V. Ramamurthy, K.S. Schanze (Eds.) Molecular and Supramolecular Photochemistry, Marcel Dekker, 2000, pp. 143-251.

[140] C.-H. Tung, K. Song, L.-Z. Wu, H.-R. Li, L.-P. Zhang, Microreactor-controlled product selectivity in organic photochemical reactions, in: V. Ramamurthy, K.S. Schanze (Eds.) Molecular and Supramolecular Photochemistry, Macel Dekker, 2001, pp. 317-383.

[141] C.-H. Tung, L.-Z. Wu, L.-P. Zhang, B. Chen, Supramolecular systems as microreactors: control of product selectivity in organic phototransformation, Accounts Chem. Res., 36 (2003) 39-47. 
[142] J.K. Thomas, Physical aspects of radiation-induced processes on $\mathrm{SiO} 2$, gamma$\mathrm{Al}_{2} \mathrm{O}_{3}$, zeolites, and clays, Chem. Rev., 105 (2005) 1683-1734.

[143] M.G.B. Rosenberg, U. H., Carbenes generated within cyclodextins and zeolites, Advances in Physical Organic Chemistry, Elsevier B. V., 40 (2005) 1-47.

[144] U.H. Brinker, M.G. Rosenberg, Carbenes generated within cyclodextrins and zeolites, in: J.P. Richard (Ed.) Advances in Physical Organic Chemistry, Elsevier B.V., 2005, pp. 1-47.

[145] S. Hashimoto, Time-resolved spectroscopic studies of aromatic species included in zeolites, in: V. Ramamurthy, K.S. Schanze (Eds.) Molecular and Supramolecular Photochemistry, Marcel Dekker, 2000, pp. 253-294.

[146] S. Hashimoto, Zeolite photochemistry: impact of zeolites on photochemistry and feedback from photochemistry to zeolite science, J. Photoch. Photobio. C, 4 (2003) 19-49.

[147] V. Ramamurthy, P. Lakshminarasimhan, C.P. Grey, L.J. Johnston, Energy transfer, proton transfer and electron transfer reactions within zeolites, Chem. Commun., (1998) 2411-2424.

[148] G. Schulz-Ekloff, D. Wohrle, B. Duffel, R.A. Schoonheydt, Chromophores in porous silicas and minerals: preparation and optical properties. Micropor. Mesopor. Mat., 51 (2002) 91-138.

[149] D.G. Shchukin, D.V. Sviridov, Photocatalytic processes in spatially confined micro- and nanoreactors, J. Photoch. Photobio. C, 7 (2006) 23-39.

[150] J.K. Thomas, E.H. Ellison, Various aspects of the constraints imposed on the photochemistry of systems in porous silica, Adv. Colloid Interfac., 89-90 (2001) 195-238.

[151] K. Takagi, T. Shichi, Photophysics and Photochemistry in Clay Minerals, in: V. Ramamurthy, K.S. Schanze (Eds.) Molecular and Supramolecular Photochemistry, Marcel Dekker, 2000, pp. 31-110.

[152] S. Takagi, M. Eguchi, D.A. Tryk, H. Inoue, Porphyrin photochemistry in inorganic/organic hybrid materials: Clays, layered semiconductors, nanotubes, and mesoporous materials, J. Photoch. Photobio. C, 7 (2006) 104-126. 
[153] S. Takagi, H. Inoue, Molecular and Supramolecular Photochemistry of Porphyrins and Metalloporphyrins, in: V. Ramamurthy, K.S. Schanze (Eds.) Molecular and Supramolecular Photochemistry, Marcel Dekker, 1999, pp. 215342.

[154] T. Shichi, K. Takagi, Clay minerals as photochemical reaction fields, J. Photoch. Photobio. C, 1 (2000) 113-130.

[155] M. Ogawa, Photoprocesses in mesoporous silicas prepared by a supramolecular templating approach, J. Photoch. Photobio. C, 3 (2002) 129-146.

[156] M. Ogawa, K. Kuroda, Photofunctions of intercalation compounds, Chem. Rev., 95 (1995) 399-438.

[157] C.V. Kumar, Photoprocesses in organized biological media in: V. Ramamurthy (Ed.) Photochemistry in organized and constrained media, VCH Publishers, Inc., 1991, pp. 783-816.

[158] C.V. Kumar, B.B. Raju, Photoactive layered materials: assembly of ions, molecules, metal complexes, and proteins, in: V. Ramamurthy, K.S. Schanze (Eds.) Molecular and Supramolecular Photochemistry, Marcel Dekker, 2001, pp. 505-576.

[159] W. Jones, Photochemistry and photophysics in clays and other layered solids, in: V. Ramamurthy (Ed.) Photochemistry in organized and constrained media, VCH Publishers, Inc., 1991, pp. 387-428.

[160] R. Dabestani, M.E. Sigman, Spectroscopy and photochemical transformations of polycylic aromatic hydrocarbons at silica- and alumina-air interfaces, in: $\mathrm{V}$. Ramamurthy, K.S. Schanze (Eds.) Molecular and Supramolecular Photochemistry, Marcel Dekker, 2000, pp. 1-30.

[161] F.L. Arbeloa, V.M. Martinez, T. Arbeloa, I.L. Arbeloa, Photoresponse and anisotropy of rhodamine dye intercalated in ordered clay layered films, J. Photoch. Photobio. C, 8 (2007) 85-108.

[162] D. Avnir, M. Ottolenghi, Photoprocesses in environment of complex geometry: fractal and porous materials, in: V. Ramamurthy (Ed.) Photochemistry in organized and constrained media, VCH Publishers, Inc., 1991, pp. 535-562. 
[163] L.J. Johnston, Phototransformations of organic molecules adsorbed on silica and alumina, in: V. Ramamurthy (Ed.) Photochemistry in organized and constrained media, VCH Publishers, Inc., 1991, pp. 359-386.

[164] V. Ramamurthy, Photoprocesses of host-guest complexes in the solid state, in: V. Ramamurthy (Ed.) Photochemistry in organized and constrained media, $\mathrm{VCH}$ Publishers, Inc., 1991, pp. 303-358.

[165] L.S. Shimizu, S.R. Salpage, A.A. Korous, Functional materials from selfassembled bis-urea macrocycles, Accounts Chem. Res., 47 (2014) 2116-2127.

[166] D.F. Eaton, Modification of optical properties of organic molecules by incorportaion into inclusion complexes, in: T.E. Mallouk (Ed.) Advances in the synthesis and reactivity of solids, Jai Press Lts, London, 1991, pp. 81-132.

[167] N.J. Turro, B. Kraeutler, Magnetic isotope and magnetic field effects on chemical reactions. Sunlight and soap for the efficient separation of carbon-13 and carbon-12, J. Am. Chem. Soc., 100 (1978) 7432-7434.

[168] D.G. Whitten, Photochemistry and photophysics of trans-stilbene and related alkenes in surfactant assemblies, Accounts Chem. Res., 26 (1993) 502-509.

[169] S. Devanathan, M.S. Syamala, V. Ramamurthy, Photoreactions in hydrophobic pockets, P. Indian As- Chem Sci., 98 (1987) 391-407.

[170] G. von Bunau, T. Wolff, Photochemistry in surfactant solutions, in: D. Volman, G. Hammond, K. Gollnick (Eds.) Advances in photochemistry, John Wiley \& Sons, 1988, pp. 273-331.

[171] M.S. Syamala, G.D. Reddy, B.N. Rao, V. Ramamurthy, Chemistry in cavities, Curr. Sci. , 55 (1986) 875-886.

[172] V. Ramamurthy, D.F. Eaton, Photochemistry and photophysics within cyclodextrin cavities, Accounts. Chem. Res. , 21 (1988) 300-306.

[173] P. Bortolus, S. Monti, Photochemistry in Cyclodextrin Cavities, in: D.C. Neckers, D.H. Volman, G.V. Bunau (Eds.) Advances in Photochemistry, 1996, pp. 1-132.

[174] K. Takahashi, Organic reactions mediated by cyclodextrins, Chem. Rev., 98 (1998) 2013-2033. 
[175] P. Bortolus, G. Graber, G. Kohler, S. Monti, Photochemistry of cyclodextrin host-guest complexes, Coordin. Chem. Rev., 125 (1993) 261-268.

[176] J.M. Haider, Z. Pikramenou, Photoactive metallocyclodextrins: sophisticated supramolecular arrays for the construction of light activated miniature devices, Chem. Soc. Rev., 34 (2005) 120-132.

[177] S. Monti, S. Sortino, Photoprocesses of photosensitizing drugs within cyclodextrin cavities, Chem. Soc. Rev., 31 (2002) 287-300.

[178] B.C. Pemberton, R. Raghunathan, S. Volla, J. Sivaguru, From containers to catalysts: supramolecular catalysis within cucurbiturils, Chem. Eur. J. , 18 (2012) 12178-12190.

[179] A. Ayitou, B.C. Pemberton, E. Kumarasamy, N. Vallavoju, J. Sivaguru, Fun with photons: selective light induced reactions in solution and in water soluble nanocontainers., Chimia, 65 (2011) 202-209.

[180] N. Vallavojo, J. Sivaguru, Supramolecular Photocatalysis: combining confinement and non-covalent interactions to control light initiated reactions, Chem. Soc. Rev., 43 (2014) 4084-4101.

[181] T. Murase, M. Fujita, Pericyclic reactions in an aqueous molecular flask, Chem. Rec., 10 (2010) 342-347.

[182] Y. Inokuma, M. Kawano, M. Fujita, Crystalline molecular flasks, Nature Chemistry, 3 (2011) 349-358.

[183] M. Yoshizawa, J.K. Klosterman, M. Fujita, Functional Molecular Flasks: new properties and reactions within discrete, self-assembled hosts, Angew. Chem. Int. Ed., 48 (2009) 3418-3438.

[184] M. Yoshizawa, M. Fujita, Development of unique chemical phenomena within nanometer-sized, self-assembled coordination hosts, B. Chem. Soc. Jpn., 83 (2010) 609-618.

[185] R. Warmuth, Inner-phase stabilization of reactive intermediates, Eur. J. Org. Chem., 2001 (2001) 423-437.

[186] R. Warmuth, The Inner phase of molecular container compounds as a novel reaction environment, J. Incl. Phenom. Macro., 37 (2000) 1-38. 
[187] R. Warmuth, Reactions inside carcerands, in: U.H. Brinker, J.-L. Mieusset (Eds.) Molecular Encapsulation: Organic Reactions in Constrained Systems, John Wiley \& Sons, Ltd, 2010, pp. 227-268.

[188] J.H. Jordan, B. C. Gibb, Molecular containers assembled through the hydrophobic effect, Chem. Soc. Rev., Advance Article (2015).

[189] V. Ramamurthy, A. Parthasarathy, Chemistry in restricted spaces: select photodimerizations in cages, cavities, and capsules, Isr. J. Chem., 51 (2011) 817829.

[190] L.S. Kaanumalle, A. Natarajan, K. Sivasubramanian, R. Kaliappan, M. Pattabiraman, V. Ramamurthy, Bioinspired-green-supramolecular-nano photochemistry: photoproducts control through weak intermolecular forces, Spectrum, 19 (2006) 16-21.

[191] V. Ramamurthy, S. Gupta, Supramolecular photochemistry: from molecular crystals to water-soluble capsules, Chem. Soc. Rev., 44 (2015) 119-135.

[192] N.J. Turro, Influence of nuclear spin on chemical reactions: Magnetic isotope and magnetic field effects., P. Natl. Acad. Sci. USA, 80 (1983) 609-621.

[193] I.R. Gould, N.J. Turro, M.B. Zimmt, Magnetic field and magnetic isotope effects on the product of organic reactions, in: V. Gold, D. Bethell (Eds.) Advances in Physical Organic chemistry, Academic Press, London, 1984, pp. 1-53.

[194] N.J. Turro, Supramolecular organic and inorganic photochemistry: Radical pair recombination in miselles, electron transfer on starburst dendrimers, and the use of DNA as a molecular wire, Pure Appl. Chem., 67 (1995) 199-208.

[195] N. J. Turro, Supramolecular Organic Photocchemistry: Control of covalent bond formation through noncovalent supramolecular interactions and magnetic effects., P. Natl. Acad. Sci. USA., 99 (2002) 4805-4809.

[196] N.J. Turro, Molecular structure as a blueprint for supramolecular structure chemistry in confined spaces, P. Natl. Acad. Sci. U.S.A., 102 (2005) 1076610770.

[197] N.J. Turro, Fun with photons, reactive intermediates, and friends. Skating on the edge of the paradigms of physical organic chemistry, organic supramolecular photochemistry and spin chemistry, J. Org. Chem., 76 (2011) 9863. 
[198] H. Dodziuk, Introduction to supramolecular chemistry, Kluwer Academic Publishers Norwell, 2002.

[199] U.H. Brinker, J.-L. Mieusset, Molecular Encapsulation, in, John Wiley \& Sons, Chichester, 2010.

[200] Z.V. Todres, Organic chemistry in confining media, Springer, Heidelberg, 2013.

[201] N. J. Turro, V. Ramamurthy, J.C. Scaiano, Modern molecular photochemistry of organic molecules, University Science Books, Sausalito, CA, 2010.

[202] B. R. Bhogala, B. Captain, A. Parthasararthy, V. Ramamurthy, Thiourea as a template for photodimerization of azastilbenes, J. Am. Chem. Soc, 132 (2010) 13434-13442.

[203] B. R. Bhogala, B. Captain, V. Ramamurthy, Comparison of templating abilities of urea and thioruea during photodimerization of bipyridylethyelene and stilbazole crystals, Photochem. Photobiol., Advance Article (2014).

[204] N. Berenjian, P.D. Mayo, M.-E. Sturgeon, L.K. Sydnes, L.C. Weedon, Biphasic photochemistry: micelle solutions as media for photochemical cycloadditions of enones, Can. J. Chem., 60 (1982) 425-436.

[205] P. De Mayo, L.K. Sydnes, Biphasic photochemistry: micellar control of regioselectivity in enone photoannulations, J. Chem. Soc., Chem. Commun. , (1980) 994-995.

[206] K. Lee, P. de Mayo, Biphasic Photochemistry: Micellar regioselectivity in enone dimerisation, J. Chem. Soc., Chem. Commun. , (1979) 493-495.

[207] T. Tamaki, T. Kokubu, K. Ichimura, Regio- and stereoselective photodimerization of anthracene derivatives included by cyclodextrins, Tetrahedron, 43 (1987) 1485-1494.

[208] J.N. Moorthy, R.G. Weiss, K. Venkatesan, Photodimerization of coumarins in solid cyclodextrin inclusion complexes J. Org. Chem., 57 (1992) 3292-3297.

[209] W. Herrmann, S. Wehrle, G. Wenz, Supramolecular control of the photochemistry of stilbenes by cyclodextrins, Chem. Commun., (1997) 17091710. 
[210] H.S. Banu, A. Lalitha, K. Pitchumani, C. Srinivasan, Modification of photochemical reactivity of trans-2-styrylpyridine: effect of cyclodextrin complexation, Chem. Commun., (1999) 607-608.

[211] M. Pattabiraman, A. Natarajan, L.S. Kaanumalle, V. Ramamurthy, Templating photodimerization of trans-cinnamic acids with cucurbit[8]uril and $\gamma$ cyclodextrin, Org. Lett., 7 (2005) 529-532.

[212] S.Y. Jon, Y.H. Ko, S.H. Park, H.-J. Kim, K. Kim, A facile, stereoselective [2+2] photoreaction mediated by cucurbit[8]uril, Chem. Commun., (2001) 1938-1939.

[213] M. Pattabiraman, A. Natarajan, R. Kaliappan, J.T. Mague, V. Ramamurthy, Template directed photodimerization of trans-1,2-bis(n-pyridyl)ethylenes and stilbazoles in water, Chem. Commun., (2005) 4542-4544.

[214] L.S. Kaanumalle, A. Natarajan, K. Sivasubramanian, R. Kaliappan, M. Pattabiraman, V. Ramamurthy, Bioinspired-green-supramolecular-nano photochemistry: Photoproducts control through weak intermolecular forces, Spectrum, 19 (2006) 16-21.

[215] M.V.S.N. Maddipatla, L.S. Kaanumalle, A. Natarajan, M. Pattabiraman, V. Ramamurthy, Preorientation of olefins toward a single photodimer: Cucurbiturilmediated photodimerization of protonated azastilbenes in water, Langmuir, 23 (2007) 7545-7554.

[216] N. Barooah, B. Pemberton, J. Sivaguru, Manipulating photochemical reactivity of coumarins within cucurbituril nanocavities, Org. Lett. 10 (2008) 3339-3342.

[217] N. Barooah, B.C. Pemberton, A.C. Johnson, J. Sivaguru, Photodimerization and complexation dynamics of coumarins in the presence of cucurbit[8]urils, Photoch. Photobio. Sci., 7 (2008) 1473-1479.

[218] B.C. Pemberton, N. Barooah, D.K. Srivatsava, J. Sivaguru, Supamolecular photocatalysis by confinement - photodimerization of coumarins within cucurbit[8]urils, Chem. Commun., 46 (2010) 225-227.

[219] B.C. Pemberton, R.K. Singh, A.C. Johnson, S. Jockusch, J.P. Da Silva, A. Ugrinov, N.J. Turro, D.K. Srivastava, J. Sivaguru, Supramolecular photocatalysis: insights into cucurbit[8]uril catalyzed photodimerization of 6methylcoumarin, Chem. Commun., 47 (2011) 6323-6325. 
[220] X.-L. Wu, L. Luo, L. Lei, G.-H. Liao, L.-Z. Wu, C.-H. Tung, Highly efficient cucurbit[8]uril-templated intramolecular photocycloaddition of 2-Naphthalenelabeled poly(ethylene glycol) in aqueous solution, J. Org. Chem., 73 (2008) 491 494.

[221] L. Lei, L. Luo, X.-L. Wu, G.-H. Liao, L.-Z. Wu, C.-H. Tung, Cucurbit[8]urilmediated photodimerization of alkyl 2-naphthoate in aqueous solution, Tetrahedron Lett., 49 (2008) 1502-1505.

[222] T. Furusawa, M. Kawano, M. Fujita, The confined cavity of a coordination cage suppresses the photocleavege of $\alpha$-diketones to give cyclization product through kinetically unfavorable pathways, Angew. Chem. Int. Ed., 46 (2007) 5717-5719.

[223] S. Horiuchi, Y. Nishioka, T. Murase, M. Fujita, Both [2+2] and [2+4] addition of inert aromatics via identical ternary host-guest complexes, Chem. Commun., 46 (2010) 3460-3462.

[224] T. Murase, S. Horiuchi, M. Fujita, Naphthalene diels-alder in a self-assembled molecular flask, J. Am. Chem. Soc., 132 (2010) 2866-2867.

[225] T. Murase, S. Peschard, S. Horiuchi, Y. Nishioka, M. Fujita, Remote chiral transfer into [2+2] and [2+4] cycloaddition within self assembled molecular flask, Supramol. Chem., 23 (2011) 199-208.

[226] Y. Nishioka, T. Yamaguchi, M. Kawano, M. Fujita, Asymmetric [2+2] olefin cross photoaddition in a self-assembled host with remote chiral auxiliaries, J. Am. Chem. Soc., 130 (2008) 8160-8161.

[227] Y. Nishioka, T. Yamaguchi, M. Yoshizawa, M. Fujita, Unusual [2+4] and [2+2] cycloadditions of arenes in the confined cavity of self-assembled cages., J. Am. Chem. Soc., 129 (2007) 7000-7001.

[228] K. Takaoka, M. Kawano, T. Ozeki, M. Fujita, Crystallographic observation of an olefin photodimerization reaction that takes place via thermal molecular tumbling within a self-assembled host., Chem. Commun., 15 (2006) 1625-1627.

[229] T. Yamaguchi, M. Fujita, Highly selective photomediated 1,4-radical addition to ortho-Quinones controlled by a self -assembled cage, Angew. Chem. Int. Ed., 47 (2008) 2067-2069. 
[230] M. Yoshizawa, Y. Takeyama, T. Kusukawa, M. Fujita, Cavity-directed, highly stereoselective [2+2] photodimerization of olefins within self-assembled coordination cages, Angew. Chem. Int. Ed., 41 ( 2002) 1347-1349.

[231] M. Yoshizawa, Y. Takeyama, T. Okano, M. Fujita, Cavity-directed synthesis within a self-assembled coordination cage: highly selective [2+2] crossphotodimerizations of olefins, J. Am. Chem. Soc., 125 (2003) 3243-3247.

[232] S. Karthikeyan, V. Ramamurthy, Templating photodimerization of coumarins within a water-soluble nano reaction vessel, J. Org. Chem., 71 (2006) 64096413.

[233] S. Karthikeyan, V. Ramamurthy, Templating photodimerization of transcinnamic acid esters with a water-soluble Pd nanocage, J. Org. Chem. , 72 (2007) 452-458.

[234] N.J. Turro, W.R. Cherry, Photoreactions in detergent solutions. Enhancement of regioselectivity resulting from the reduced dimensionality of substrates sequestered in a micelle, J. Am. Chem. Soc., 100 (1978) 7431-7432.

[235] N.J. Turro, G.C. Weed, Micellar systems as "supercages" for reactions of geminate radical pairs. Magnetic effects, J. Am. Chem. Soc., 105 (1983) 18611868.

[236] B.H. Baretz, N.J. Turro, Photochemistry of diastereomeric 2,4-diphenylpentan-3ones and related ketones in "super cage" environments provided by micelles, porous glass, and porous silica: Temperature and magnetic field effects, J. Am. Chem. Soc., 105 (1983) 1309-1316.

[237] B. Kraeutler, N.J. Turro, Probes for the micellar cage effect. The Magnetic 13Cisotope effect and a new cage product in the photolysis of dibenzyl ketone, Chem. Phys. Lett., 70 (1980) 270-275.

[238] N.J. Turro, M.-F. Chow, C.-J. Chung, B. Kraeutler, Magnetic and micellar effects on photoreactions. I. ${ }^{13} \mathrm{C}$ isotopic enrichment of dibenzyl ketone via photolysis in aqueous detergent solution, J. Am. Chem. Soc., 103 (1981) 38863891.

[239] N.J. Turro, D.R. Anderson, M.-F. Chow, C.-J. Chung, B. Kraeutler, Magnetic and micellar effects on photoreactions. 2. Magnetic isotope effects on quantum 
yields and magnetic field effects on separation efficiency. Correlation of ${ }^{13} \mathrm{C}$ enrichment parameters with quantum yield measurements, J. Am. Chem. Soc., 103 (1981) 3892-3896.

[240] D.J. Cram, J.M. Cram, Container molecules and their guests, Royal Society of Chemistry, Cambridge, 1997.

[241] D.J. Cram, M.E. Tanner, R. Thomas, The taming of cyclobutadiene, Angew. Chem., Int. Ed., 30 (1991) 1024-1027.

[242] R. Warmuth, o-Benzyne: Strained alkyne or cumulene?-NMR characterization in a molecular container, Angew. Chem. Int. Ed. Engl., 36 (1997) 1347-1350.

[243] V.P. Rao, M.B. Zimmt, N.J. Turro, Photoproduction of remarkably stable benzylic radicals in cyclodextrins inclusion complexes, J. Photoch. Photobio., A, 60 (1991) 355-360.

[244] R. Choudhury, S. Gupta, J.P. Da Silva, V. Ramamurthy, Deep-cavity cavitand octa acid as a hydrogen donor: photofunctionalization with nitrenes generated from azidoadamantanes, J. Org. Chem., 78 (2013) 1824-1832.

[245] S. Gupta, R. Choudhury, D. Krois, U.H. Brinker, V. Ramamurthy, Cucurbituril adamantanediazirine complexes and consequential carbene chemistry, J. Org. Chem., 77 (2012) 5155-5160.

[246] S. Gupta, R. Choudhury, D. Krois, G. Wagner, U.H. Brinker, V. Ramamurthy, Photochemical generation and reactivity of carbenes within an organic cavitand and capsule: Photochemistry of adamantanediazirines, Org. Lett., 13 (2011) 6074-6077.

[247] Z. Lu, R.A. Moss, R.R. Sauers, R. Warmuth, Innermolecular reactions of fluorophenylcarbene inside a hemicarcerand, Org. Lett., 11 (2009) 3866-3869.

[248] X.C. Liu, G, ; Moss, R. A. ; Sauers, R. R. ; Warmuth, R., Fluorophenoxycarbene inside a hemicarcerand: A bottled singlet carbene, Angew. Chem. Int. Ed., 44 (2005) 1994-1997.

[249] Z.M. Lu, R. A. ; Sauers, R. R. ; Warmuth, R., Innermolecular reactions of fluorophenylcarbene inside a hemicarcerand, Org. Lett., 11 (2009) 3866-3869. 
[250] R. Warmuth, S. Makowiec, Photochemical and thermal reactions of intermediates in the phenylnitirne rearrangement inside a hemicarcerand, J. Am. Chem. Soc., 129 (2007) 1233-1241.

[251] W. Kirmse, Incarcerated carbenes, Angew. Chem. Int. Ed., 44 (2005) 2476-2479.

[252] U.H. Brinker, R. Buchkremer, M. Kolodziejczyk, R. Kupfer, M. Rosenberg, M.D. Poliks, M. Orlando, M.L. Gross, Carbenes in constrained systems I: 1,3 C$\mathrm{H}$, insertion reaction of adamantylidene within the $\beta$-Cyclodextrin cavity, Angew. Chem. Int. Ed., 32 (1993) 1344-1345.

[253] U.H. Brinker, P. Walla, D. Krois, V.B. Arion, Study of the structure and photochemical decomposition of azidoadamantanes entrapped in $\alpha$ - and $\beta$ cyclodextrin, Eur. J. Org. Chem., 2011 (2011) 1249-1255.

[254] J.-L. Mieusset, U.H. Brinker, Encapsulation of reactive intermediates, in: molecular encapsulation: organic reactions in constrained systems, Wiley, Chichester, West sussex, 2010, pp. 269-308.

[255] J.V. Caspar, V. Ramamurthy, D.R. Corbin, Preparation and spectroscopic characterization of polarons and bipolarons of thiophene oligomers within the channels of pentasil zeolites: The evolution of organic radical ions into conducting polymers, J. Am. Chem. Soc., 113 (1991) 600-610.

[256] V. Ramamurthy, J.V. Caspar, D.R. Corbin, Generation, entrapment, and spectroscopic characterization of radical cations of .alpha.,.omega.-diphenyl polyenes within the channels of pentasil zeolites, J. Am. Chem. Soc., 113 (1991) 594-600.

[257] V. Ramamurthy, R.G. Weiss, G.S. Hammond, A model for the influence of organized media on photochemical reactions, in: D. Volman, G.S. Hammond, D.C. Neckers (Eds.) Advances in photochemistry, John Wiley \& sons., 1993, pp. 67-234.

[258] R.G. Weiss, V. Ramamurthy, G.S. Hammond, Photochemistry in organized and confining media: A model, Accounts Chem. Res., 26 (1993) 530-536.

[259] N. J. Turro, M. Garcia-Garibay, Thinking topologically about photochemistry in restricted spaces, in: V. Ramamurthy (Ed.) Photochemistry in organized and constrained media, VCH Publishers, Inc., 1991, pp. 1-38. 
[260] N. Ramasubbu, K. Gnanaguru, K. Venkatesan, V. Ramamurthy, A study on the photochemical dimerizations of coumarins in the solid state, J. Org. Chem., 50 (1985) 2337.

[261] G.S. Murthy, P. Arjunan, K. Venkatesan, V. Ramamurthy, Consequences of lattice relaxability in solid state photodimerizations, Tetrahedron, 43 (1987) 1225-1240.

[262] G.M.J. Schmidt, Topochemistry part III. The crystal chemistry of some transcinnamic acids, J. Chem. Soc., (1964) 21-28.

[263] M.D. Cohen, G.M. Schmidt, F.I. Sonntag, Topochemistry. Part II. The photochemistry of trans-cinnamic acids, J. Chem. Soc., (1964) 2000-2013.

[264] K. Kalyanasundaram, F. Grieser, J.K. Thomas, Room temperature phosphorescence of aromatic hydrocarbons in aqueous micellar solutions, Chem. Phys. Lett., 51 (1977) 501-505.

[265] N. J. Turro, K. Liu, M. Chow, P. Lee, Convenient and simple methods for the observation of phosphorescence in fluid solutions: internal and external heavy atom and micellar effects, Photochem. Photobiol., 27 (1978) 523-529.

[266] S. Hamai, Room-temperature phosphorescence from 1:1:1 inclusion compounds of $\beta$-Cyclodextrin with brominated alcohols and acenaphthene, J. Am. Chem. Soc., 111 (1989) 3954-3957.

[267] S. Hamai, Inclusion complexes and the room-temperature phosphorescence of 6bromo-2-naphthol in aerated aqueous solution of $\alpha$-Cyclodextrin, J. Phys. Chem., 99 (1995) 12109-12114.

[268] A. Ponce, P.A. Wong, J.J. Way, D.G. Nocera, Intense phosphorescence triggered by alcohols upon formation of a cyclodextrin ternary complex, J. Phys. Chem., 97 (1993) 11137-11142.

[269] C.M. Rudzinski, D.G. Nocera, Buckets of Light, in: V. Ramamurthy, K.S. Schanze (Eds.) Molecular and Supramolecular Photochemistry, Marcel Dekker, 2001, pp. 1-91.

[270] S. Scypinski, C.L.J. Love, Room-temperature phosphorescence of polynuclear aromatic hydrocarbons in cyclodextrins, Anal. Chem., 56 (1984) 322-327. 
[271] V. Ramamurthy, J.V. Caspar, D.F. Eaton, E.W. Kuo, D.R. Corbin, Heavy-atominduced phosphorescence of aromatics and olefins included within zeolites, J. Am. Chem. Soc., 114 (1992) 3882-3892.

[272] V. Ramamurthy, J. Caspar, D.R. Corbin, B.D. Schlyer, A.H. Maki, Triplet-state photophysics of naphthalene and $\alpha, \omega$-diphenylpolyenes induced in heavy-cationexchanged zeolites, J. Phys. Chem., 94 (1990) 3391-3393.

[273] I.M. Jakovljevic, Lead or thallium salts as external heavy atoms for room temperature quantitative phosphorescence, Anal. Chem., 49 (1977) 2048-2050.

[274] L.M. Perry, A.D. Campiglia, J.D. Winefordner, Room-temperature phosphorescence of polynuclear aromatic hydrocarbons on matrix-modified solid substrates, Anal. Chem., 61 (1989) 2328-2330.

[275] E.L.-Y. Bower, J.D. Winefordner, The Effect of sample environment on the room-temperature phosphorescence of several polynuclear aromatic hydrocarbons, Anal. Chim. Acta, 102 (1978) 1-13.

[276] B.B. Purdy, R.J. Hurtubise, Changes in the photophysical properties with heavy atoms and the effects of modulus for 4-Phenylphenol in solid-matrix luminescence, Appl. Spectrosc., 46 (1992) 988-993.

[277] N. Jayaraj, M.V.S.N. Maddipatla, R. Prabhakar, S. Jockusch, N.J. Turro, V. Ramamurthy, Closed nanocontainer enables thioketones to phosphoresce at room temperature in aqueous solution, J. Phys. Chem. B., 114 (2010) 14320-14328.

[278] G.S. Hammond, R.S. Cole, Asymmetric induction during energy transfer, J. Am. Chem. Soc., 87 (1965) 3256-3257.

[279] Y. Inoue, Asymmetric photochemical reactions in solution, Chem. Rev., 92 (1992) 741-770.

[280] Y. Inoue, V. Ramamurthy, Chiral photochemistry, in, Marcel Dekker, New York, 2004.

[281] H. Rau, Asymmetric photochemistry in solution, Chem. Rev., 83 (1983) 535547.

[282] H. Buschman, H.-D. Scharf, N. Hoffmann, P. Esser, The isoinversion principlea general model of chemical selectivity, Angew. Chem. Int. Ed. Engl., 30 (1991) 477-515. 
[283] S. Faure, S. Piva-le-Blanc, C. Bertrand, J.-P. Pete, R. Faure, O. Piva, Asymmetric intramolecular $2+2$ photocycloadditions: $\alpha$ and $\beta$ - hydroxyacids as chiral tether groups, J. Org. Chem., 67 (2002) 1061-1070.

[284] A. Elgavi, S. B. Green, G. M. J. Schmidt, Reactions in chiral crystals. Optically active heterophotodimer formation from chiral single crystals., J. Am. Chem. Soc., 95 (1973) 2058-2059.

[285] E. Cheung, K.C.W. Chong, S. Jayaraman, V. Ramamurthy, J.R. Scheffer, J. Trotter, Enantio- and diastereo-differentiating cis, trans-photoisomerization of 2 $\beta$, $3 \beta$-diphenylcyclopropane-1a-carboxylic acid derivatives in organized media, Org. Lett., 2 (2000) 2801-2804.

[286] K.C.W. Chong, J. Sivaguru, T. Shichi, Y. Yoshimi, V. Ramamurthy, J.R. Scheffer, Use of chirally modified zeolites and crystals in photochemical asymmetric synthesis, J. Am. Chem. Soc., 124 (2002) 2858-2859.

[287] J. N. Gamlin, R. Jones, M. Leibovitch, B. Patrick, J.R. Scheffer, J. Trotter, The ionic auxiliary concept in solid state organic photochemistry, Accounts Chem. Res., 29 (1996) 203-209.

[288] J. Chen, M. Garcia-Garibay, J.R. Scheffer, Chiral handle induced diastereoselectivity in an organic photorearrangement: solution versus solid state results., Tetrahedron Lett., 30 (1989) 6125-6128.

[289] M. Leibovitch, G. Olovsson, G. Sundarababu, V. Ramamurthy, J.R. Scheffer, J. Trotter, Asymmetric induction in photochemical reactions conducted in zeolites and in the crystalline state, J. Am. Chem. Soc., 118 (1996) 1219-1220.

[290] J.R. Scheffer, A.A. Dzakpasu, Organic photochemistry in the solid state. The distance and geometric requirements for intramolecular hydrogen abstraction reactions. Structure-reactivity relationships based on X-Ray crystallographic data., J. Am. Chem. Soc., 100 (1978) 2163-2173.

[291] M. Farina, G. Audisio, G. Natta, A new kind of asymmetric synthesis. The radiation polymerization of trans-1,3-pentadiene included in optically active perhydrotriphenylene, J. Am. Chem. Soc., 89 (1967) 5071-5071.

[292] K. Tanaka, F. Toda, Solvent-free organic synthesis, Chem. Rev., 100 (2000) 1025-1074. 
[293] F. Toda, Solidstate organic chemistry: Efficient reactions, remarkable yields, and stereoselectivity, Accounts Chem. Res., 28 (1995) 480-486.

[294] A. Joy, S. Uppili, M.R. Netherton, J.R. Scheffer, V. Ramamurthy, Photochemistry of a tropolone ether and 2,2-dimethyl-1-(2H)-naphthalenones within a zeolite: enhanced diastereoselectivity via confinement., J. Am. Chem. Soc., 122 (2000) 728-729.

[295] A. Joy, J.R. Scheffer, V. Ramamurthy, Chirally modified zeolites as reaction media: Photochemistry of an achiral tropolone ether, Org. Lett., 2 (2000) 119121.

[296] A. Joy, V. Ramamurthy, J.R. Scheffer, D.R. Corbin, Enantioselective photoelectrocyclization within zeolites: tropolone methyl ether in chirally modified NaY, Chem. Commun., (1998) 1379-1380.

[297] A. Joy, R. Robbins, K. Pitchumani, V. Ramamurthy, Asymmetrically modified zeolite as a medium for enantioselective photoreactions: Reactions from spin forbidden excited states, Tetrahedron Lett., (1997) 8825-8828.

[298] S. Uppili, V. Ramamurthy, Enhanced enantio- and diastreoselctivities via confinement: photorearrangement of 2,4-cyclohexadienones included in zeolites, Org. Lett., 4 (2002) 87-90.

[299] A. Natarajan, K. Wang, V. Ramamurthy, J.R. Scheffer, B. Patrick, Control of enantioselectivity in the photochemical conversion of $\alpha$-oxoamides into $\beta$-lactam derivatives, Org. Lett., 4 (2002) 1443-1446.

[300] A. Natarajan, A. Joy, L.S. Kaanumalle, J.R. Scheffer, V. Ramamurthy, Enhanced enantio- and diastereoselectivity via confinement and cation binding: Yang photocyclization of 2-benzoyladamantane derivatives within zeolites, J. Org. Chem., 67 (2002) 8339-8350.

[301] J. Sivaguru, J.R. Scheffer, J. Chandarasekhar, V. Ramamurthy, Confined space and cations enhance the power of a chiral auxiliary: photochemistry of 1,2diphenylcyclopropane derivatives, Chem. Commun., (2002) 830-831.

[302] J. Sivaguru, J.R. Scheffer, J. Chandrasekhar, V. Ramamurthy, Confined space and cations enhance the power of a chiral auxiliary: photochemistry of 1,2diphenylcyclopropane derivatives, Chem. Commun., (2002) 830-831. 
[303] J. Sivaguru, J. Shailaja, V. Ramamurthy, Organic photochemistry within zeolites: selectivity through confinement, in: S.M. Auerbach, K.A. Carrado, P.K. Dutta (Eds.) Handbook Of Zeolite Science and Technology, Marcel Dekker, New York, 2003, pp. 515-589.

[304] J. Sivaguru, T. Shichi, V. Ramamurthy, Reactive-state spin-dependent diastereoselective photoisomerization of trans,trans -2,3-diphenylcyclopropane1-carboxylic acid derivatives included in zeolites, Org. Lett., 4 (2002) 4221 4224.

[305] J. Sivaguru, R.B. Sunoj, T. Wada, Y. Origane, Y. Inoue, V. Ramamurthy, Enhanced diastereoselectivity via confinement: diastereoselective photoisomerization of 2,3-diphenyl-1-benzoylcyclopropane derivatives within zeolites, J. Org. Chem., 69 (2004) 5528-5536.

[306] J. Sivaguru, R.B. Sunoj, T. Wada, Y. Origane, Y. Inoue, V. Ramamurthy, Enhanced diastereoselectivity via confinement: Diastereoselective Photoisomerization of 2,3-Diphenylcyclopropane-1-carboxylic Acid Derivatives within Zeolites, J. Org. Chem., 69 (2004) 6533-6547.

[307] J. Sivaguru, T. Wada, Y. Origane, Y. Inoue, V. Ramamurthy, Mechanism of photoisomerization of optically pure trans-2,3-diphenylcyclopropane-1carboxylic acid derivatives, Photoch.Photobio. Sci., 4 (2005) 119-127.

[308] K. Sivasubramanian, L.S. Kaanumalle, S. Uppili, V. Ramamurthy, Value of zeolites in asymmetric induction during photocyclization of pyridones, cyclohexadienones and naphthalenones, Org. Biomol. Chem., 5 (2007) 15691576.

[309] S. Koodanjeri, A. Joy, V. Ramamurthy, Asymmetric induction with cyclodextrins : photocyclization of tropolone alkyl ethers, Tetrahedron, 56 (2000) 7003-7009.

[310] S. Jayaraman, S. Uppili, A. Natarajan, A. Joy, K.C.W. Chong, M.R. Netherton, A. Zenova, J.R. Scheffer, V. Ramamurthy, The influence of chiral auxiliaries is enhanced within zeolites, Tetrahedron Lett., 41 (2000) 8231-8235. 
[311] H. Takeshita, M. Kumamoto, I. Kouno, Synthetic Photochemistry. XIII Chiroptical retention for the reported "antara-anatra"-3-3-sigmatropy of bicyclo[3.2.0]hepta3,6-dien-2-ones, Bull. Chem. Soc. Jpn., 53 (1980) 1006-1009.

[312] H. Aoyama, K.-I. Miyazaki, M. Sakamoto, Y. Omote, Solid state photoreaction of N,N-dialkylpyruvamides: Inclusion complexes with deoxycholic acid or cyclodextrin, Tetrahedron, 43 (1987) 1513-1518.

[313] V.P. Rao, N.J. Turro, Asymmetric induction in benzoin by photolysis of benzaldehyde absorbed in cyclodextrin cavities, Tetrahedron Lett., 30 (1989) 4641-4644.

[314] L. Weber, I. Imiolczyk, G. Haufe, D. Rehorek, H. Hennig, Photocatalytic enantiodiscriminating oxygenation with cyclodextrin-linked porphyrins and molecular oxygen, Chem. Commun. , (1992) 301-303.

[315] S. Koodanjeri, A. Joy, V. Ramamurthy, Asymmetric induction with cyclodextrins: photocyclization of tropolone alkyl ethers, Tetrahedron, 56 (2000) 7003-7009.

[316] S. Koodanjeri, V. Ramamurthy, Cyclodextrin mediated enantio and diastereoselective geometric photoisomerization of diphenylcyclopropane and its derivatives, Tetrahedron Lett., 43 (2002) 9229-9232.

[317] J. Shailaja, S. Karthikeyan, V. Ramamurthy, Cyclodextrin mediated solvent-free enantioselective photocyclization of $\mathrm{N}$-alkyl pyridones, Tetrahedron Lett., 43 (2002) 9335-9339.

[318] K. Vizvardi, K. Desmet, I. Luyten, P. Sandra, G. Hoornaert, E.V.D. Eycken, Asymmetric induction in intramolecular meta photocycloaddition: Cyclodextrinmediated solid-phase photochemistry of various phenoxyalkenes., Org. Lett., 3 (2001) 1173-1175.

[319] A. Nakamura, Y. Inoue, Supramolecular Catalysis of the enantiodifferentiating [4+4] photocyclodeimerization of 2-anthracenecarboxylate by $\gamma$-Cyclodextrin, J. Am. Chem. Soc., 125 (2003) 966-972.

[320] C. Yang, M. Nishijima, A. Nakamura, T. Mori, T. Wada, Y. Inoue, A remarkable stereoselectivity switching upon solid-state versus solution-phase enantiodifferentiating photocyclodimerization of 2-anthracenecarboxylic acid 
mediated by native and 3,6-anhydro-gamma-cyclodextrins, Tetrahedron Lett., 48 (2007) 4357-4360.

[321] L. Luo, G.-H. Liao, X.-L. Wu, L. Lei, C.-H. Tung, L.-Z. Wu, gammaCyclodextrin-directed enantioselective photocyclodimerization of methyl 3methoxyl-2-naphthoate, J. Org. Chem., 74 (2009) 3506-3515.

[322] L. Luo, S.-F. Cheng, B. Chen, C.-H. Tung, L.-Z. Wu, Stepwise photochemicalchiral delivery in gamma-cyclodextrine-directed enantioselective photocyclodimerization of methyl 3-methoxyl-2-naphthoate in aqueous solution, Langmuir, 26 (2010) 782-785.

[323] H.-X. Xu, S.-F. Cheng, X.-J. Yang, B. Chen, Y. Chen, L.-P. Zhang, L.-Z. Wu, W. Fang, C.-H. Tung, R.G. Weiss, Enhancement of diastereoselectivity in photodimerization of alkyl 2-naphthoates with chiral auxiliaries via inclusion within gamma-cyclodextrin cavities, J. Org. Chem., 77 (2012) 1685-1692.

[324] G. Fukuhara, H. Umehara, S. Higashino, M. Nishijima, C. Yang, T. Mori, T. Wada, Y. Inoue, Supramolecular photocyclodimerization of 2hydroxyanthracene with a chiral hydrogen-bonding template, cyclodextrin and serum albumin, Photoch. Photobio. Sci., 13 (2014) 162-171.

[325] C. Yang, Y. Inoue, Supramolecular photochirogenesis, Chem. Soc. Rev., 43 (2014) 4123-4143.

[326] C.T. Libermann, Ann. Chem. Pharm., 158 (1871) 300.

[327] C. Bohne, Supramolecular dynamics studied using photophysics, Langmuir, 22 (2006) 9100-9111.

[328] C. Bohne, Dynamics of guest binding to supramolecular assemblies, in: V. Ramamurthy, Y. Inoue (Eds.) Supramolecular Photochemistry, John Wiley\& Sons, Inc., 2011, pp. 1-52.

[329] C. Bohne, Supramolecular dynamics, Chem. Soc. Rev., 43 (2014) 4037-4050.

[330] M. Freitag, E. Galoppini, Cucurbituril complexes of viologens bound to $\mathrm{TiO}_{2}$ films, Langmuir 26 (2010) 8262-8269.

[331] M. Freitag, E. Galoppini, Molecular host-guest complexes: shielding of guests on semiconductor surfaces, Energy Environ. Sci. , 4 (2011) 2482-2494. 
[332] S.A. Haque, J.S. Park, M. Srinivasarao, J.R. Durrant, Molecular-level insulation: An approach to controlling interfacial charge transfer, Adv. Mater., 16 (2004) 1177-1181.

[333] H. Choi, S.O. Kang, J. Ko, G. Gao, H.S. Kang, M.-S. Kang, M.K. Nazeeruddin, M. Graetzel, An efficient dye-sensitized solar cell with an organic sensitizer encapsulated in a cyclodextrin cavity, Angew. Chem., Int. Ed. , 48 (2009) 59385941.

[334] Y. Ishida, R. Kulasekaran, T. Shimada, S. Takagi, V. Ramamurthy, Efficient singlet-singlet energy transfer in a novel host-guest assembly composed of an organic cavitand, aromatic molecules and clay nanosheet, Langmuir, 29 (2013) 1748-1753.

[335] E. Ramasamy, I. K. Deshapriya, R. Kulasekharan, C.V. Kumar, V. Ramamurthy, Photophysical studies of an encapsulated neutral guest intercalated into the 2-dimensional space of $\alpha-\operatorname{Zr}(\mathrm{IV})$ phosphate, Photoch. Photobio. Sci., 13 (2014) 301-309.

[336] Y. Ishida, R. Kulasekharan, T. Shimada, V. Ramamurthy, S. Takagi, Supramolecular-surface photochemistry: Supramolecular assembly organized on a clay surface facilitates energy transfer between an encapsulated donor and a free acceptor, J. Phys. Chem. C, 118 (2014) 10198-10203. 


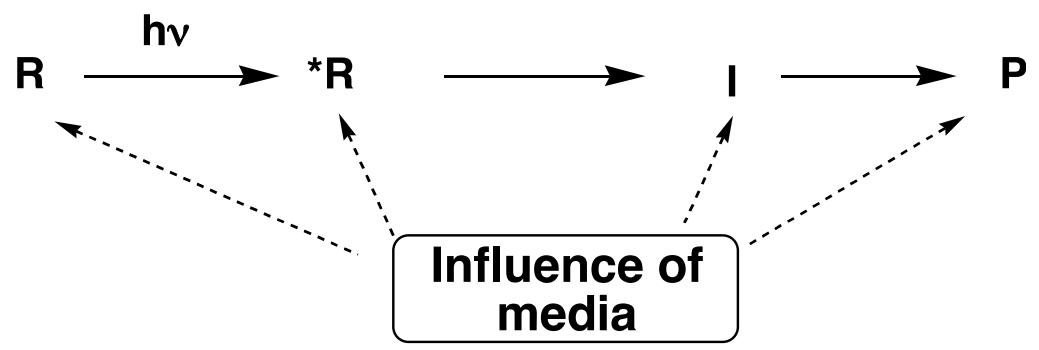

Scheme 1 Influence of media on various stages in a photochemical reaction. R, * R, I and $\mathrm{P}$ are reactant in the ground state, in the excited state, reaction intermediate, and product in the ground state respectively. 


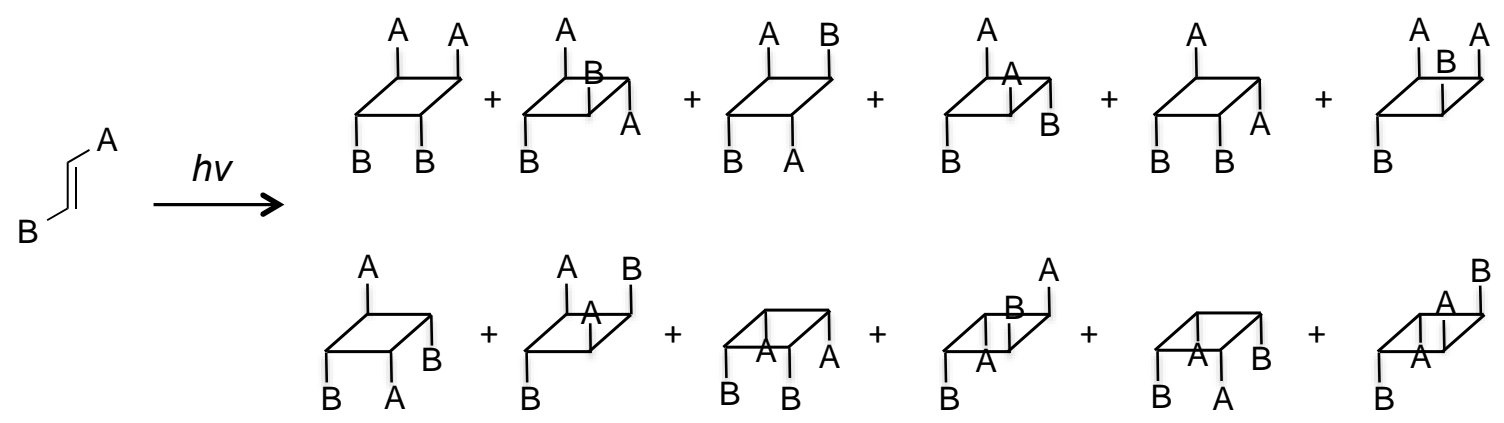

Scheme 2 Isomeric dimers possible from an olefin with two substituents, upon photodimerization. 


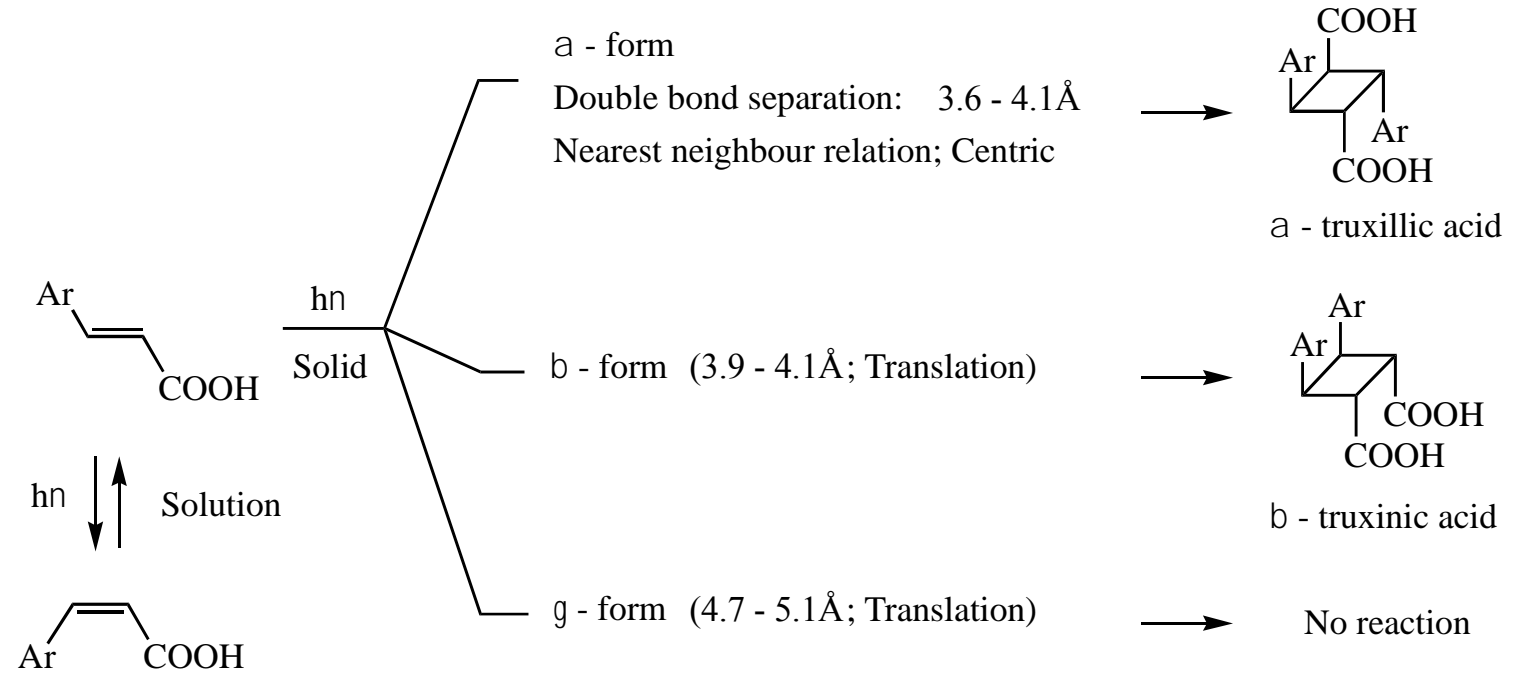

Scheme 3 Photodimerization of trans- cinnamic acid upon irradiation in solution and in solid state. The scheme illustrates the topochemical rules formulated by G. M. J. Schmidt. 


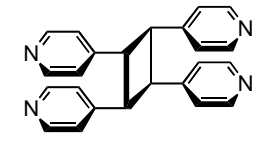

syn

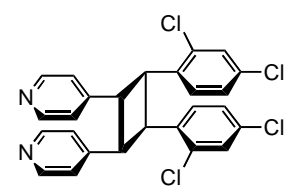

syn head-head

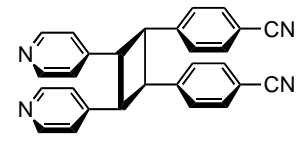

syn head-head

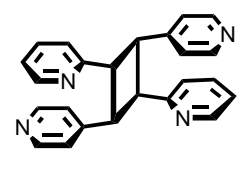

anti head-tail

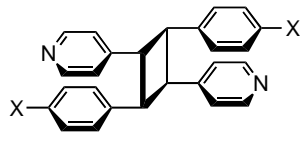

anti head-tail $\mathrm{X}=\mathrm{H}, \mathrm{F}, \mathrm{Cl}, \mathrm{Br}, \mathrm{CH}_{3}, \mathrm{OCH}_{3}$

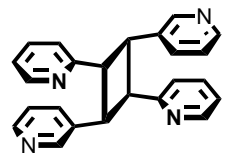

anti head-tail

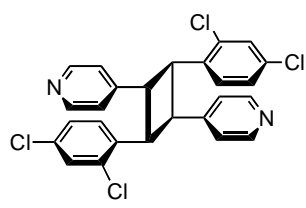

anti head-tail

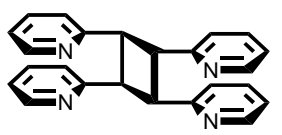

syn

Scheme 4 Various dimers obtained using thiourea as a template to pre-orient stilbazole and bispyridylethylene derivatives. 
$\underset{\mathrm{C}}{\stackrel{h v}{\longrightarrow}} \stackrel{h y s t a l}{\longrightarrow}$ No dimer
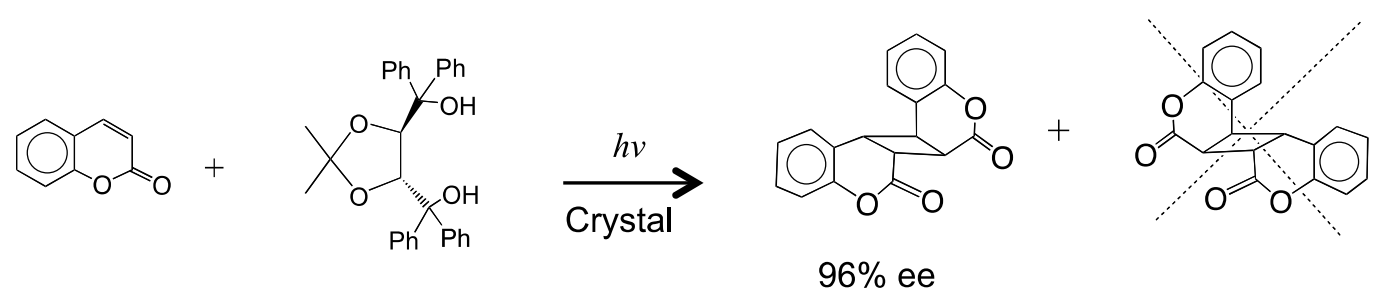

$96 \%$ ee

Scheme 5 Top: Coumarin is photostable in the crystalline state. Coumarin yields a single anti- head-head dimer when crystallized with an optically active host (template). 


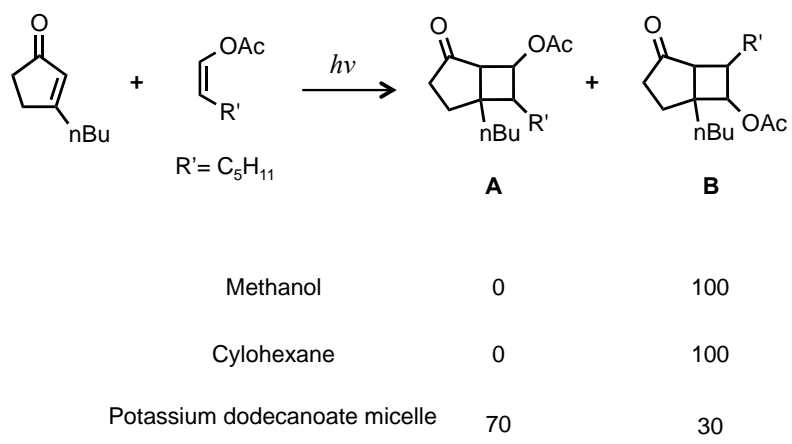

Scheme 6 Photocycloaddition of 3-n-butylcyclopentanone with heptenyl acetate in organic solvents and in potassium dodecanoate micelle. Note the ratio of the two dimers change between isotropic solvent and micelles. 


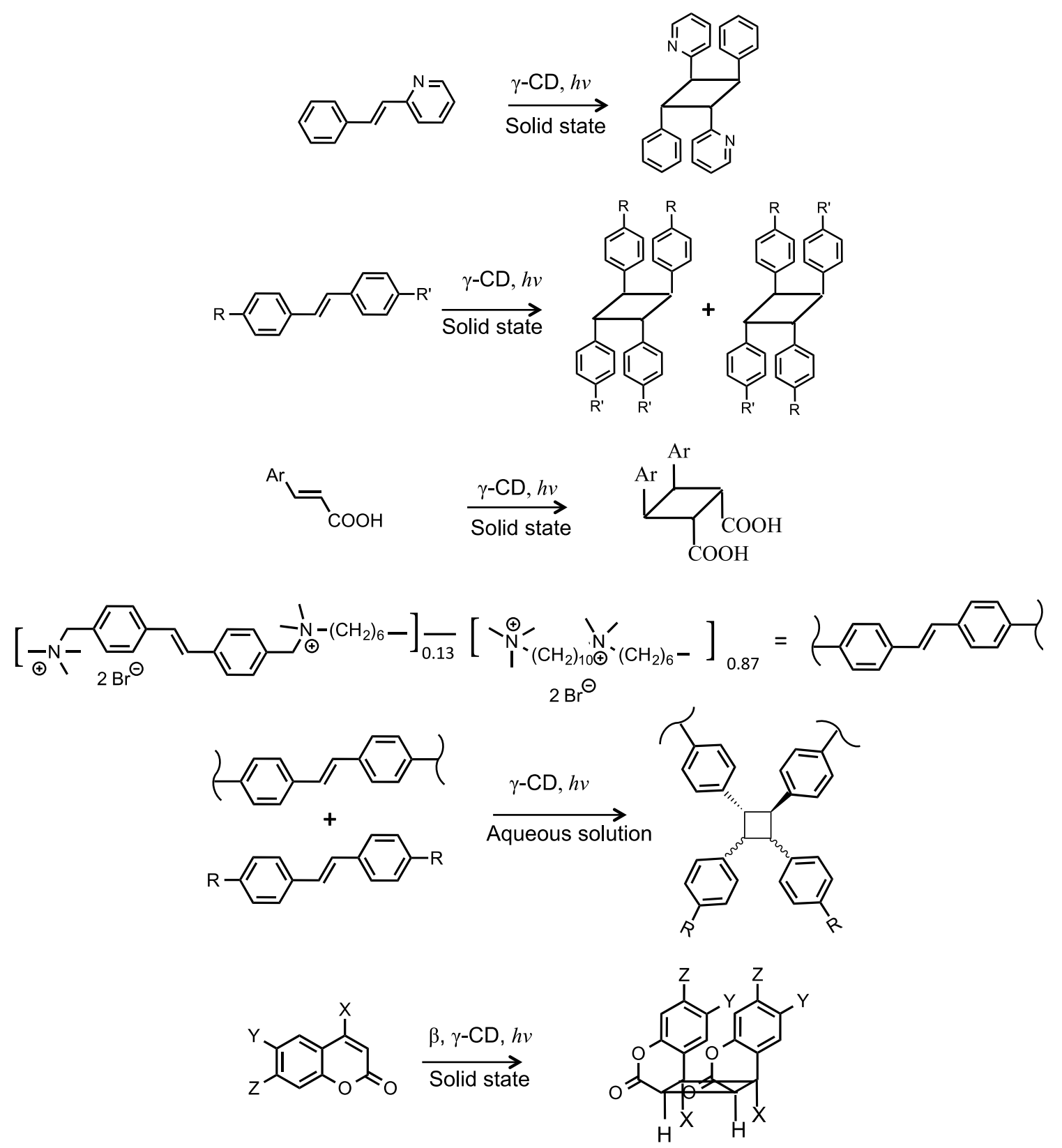

Scheme 7 Selected examples of dimerization of olefins included within cyclodextrin. Note: most studies are in solid state. Cyclodextrin complexes are not very soluble in water. 

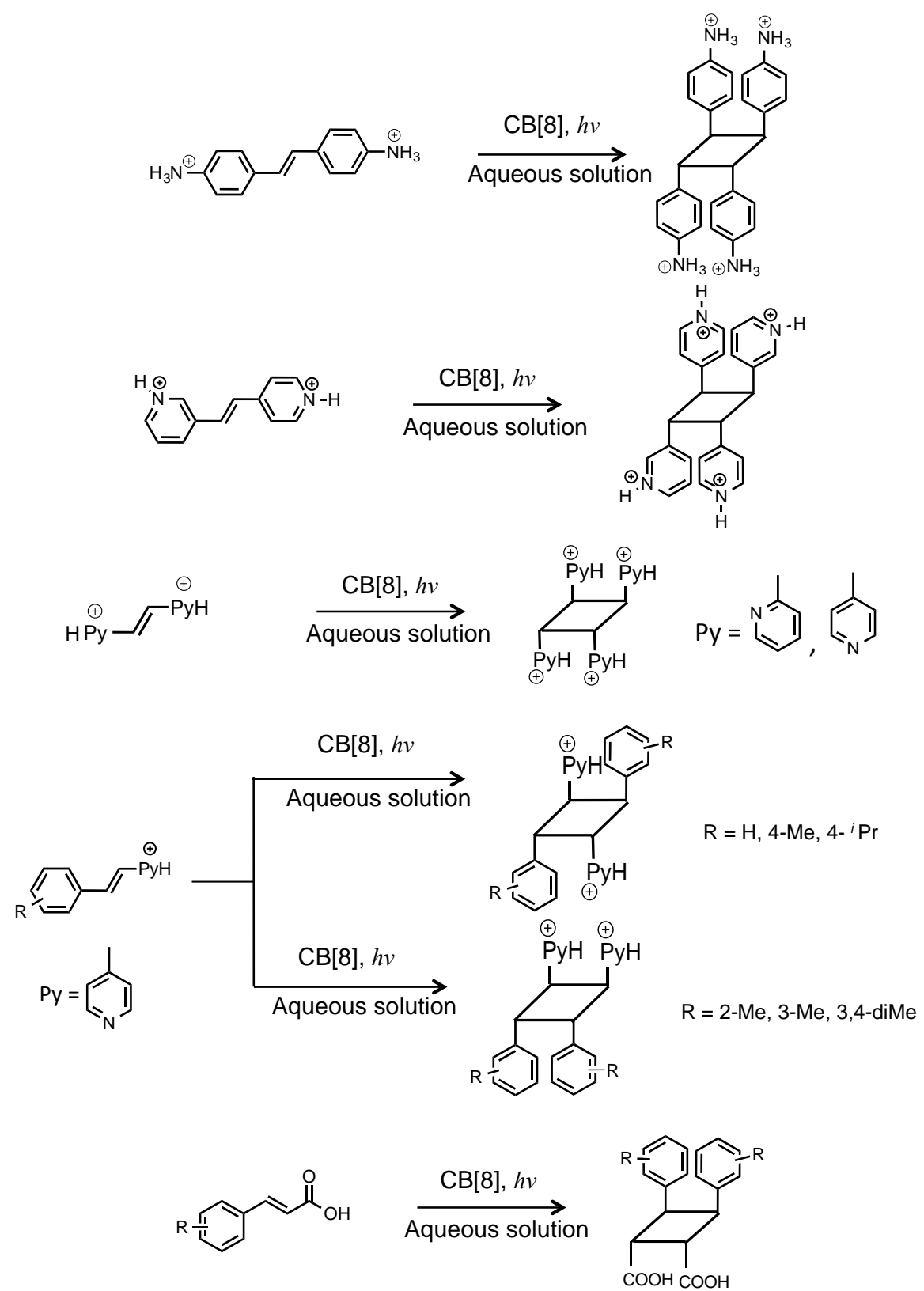

Scheme 8 Selected examples of dimerization of olefins included within cucurbit[n]uril. 


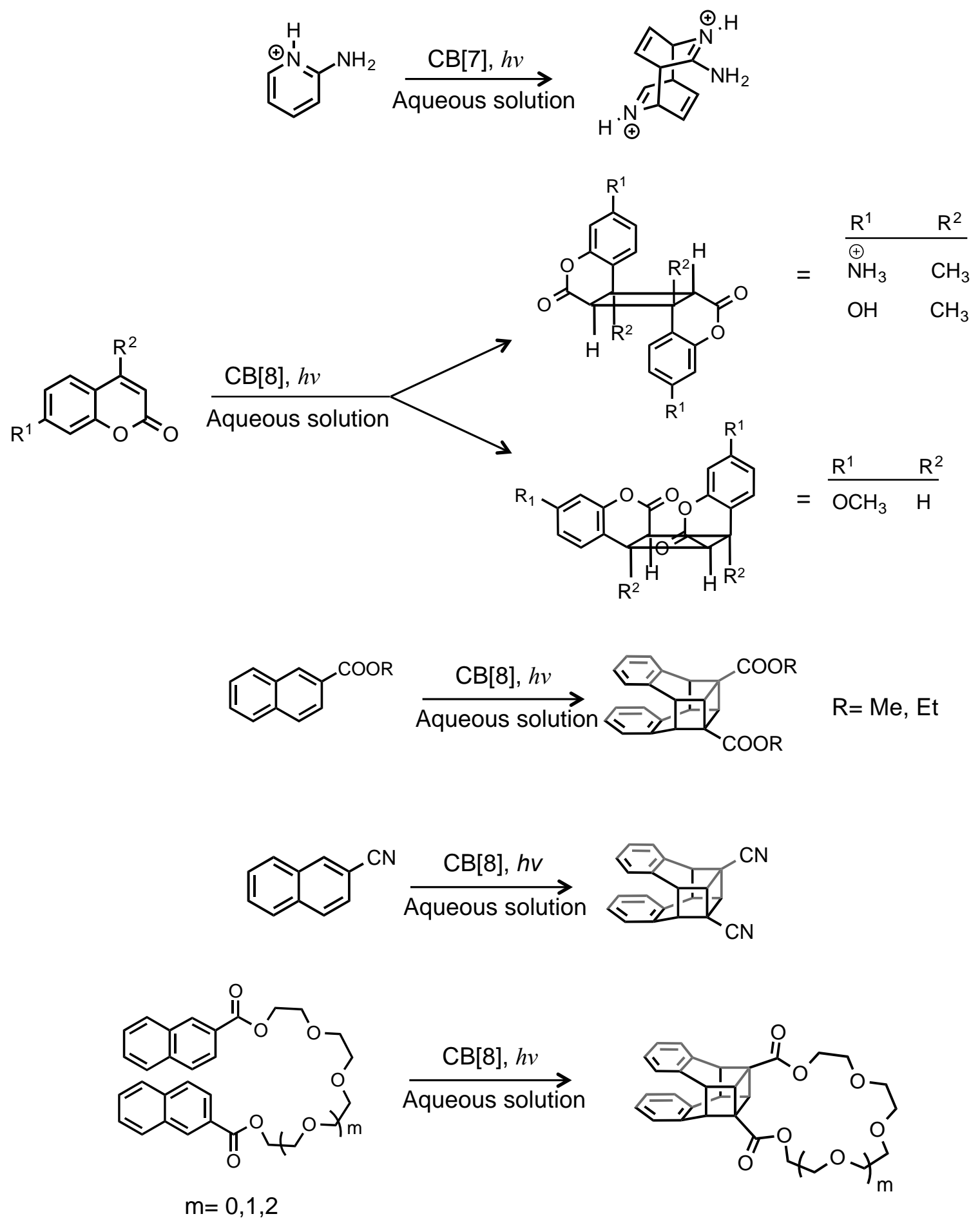

Scheme 9 Selected examples of dimerization of olefins included within cucurbit[n]uril. 


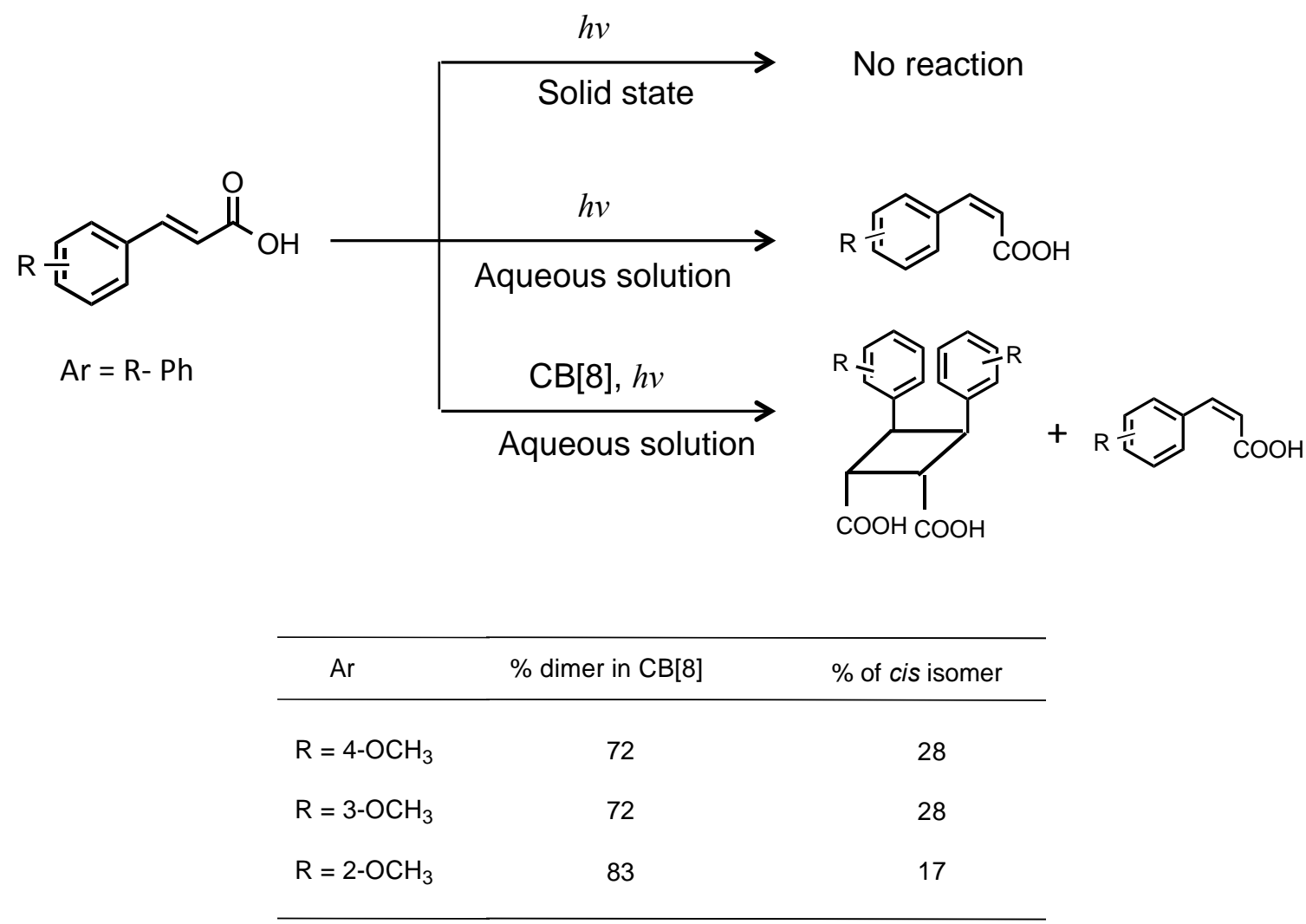

Scheme 10 Photodimerization of cinnamic acids included within cucurbit[8]uril. Yields are reported in relative mole percentage. 

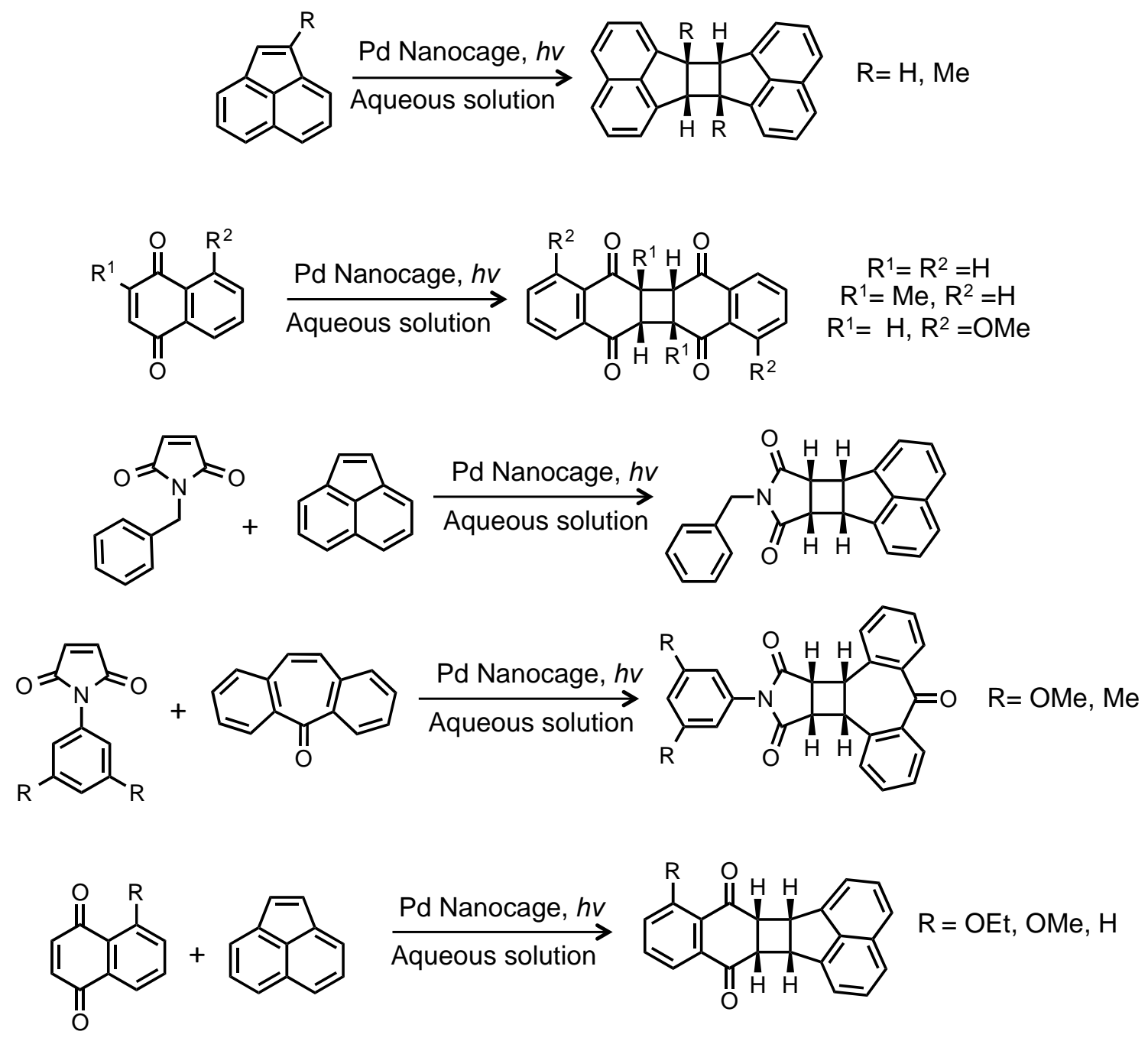

Scheme 11 Examples of photodimerization within Pd nanocage reported by Fujita group. 
<smiles>c1ccc2c(c1)c1ccccc1c1ccccc21</smiles><smiles>O=C1C=CC(=O)N1C1CCCCC1</smiles>

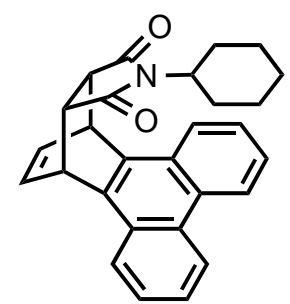<smiles>c1cc2cccc3c4cccc5ccc6cccc(c(c1)c23)c4c65</smiles><smiles>O=C1C=CC(=O)N1C1CCCCC1</smiles>

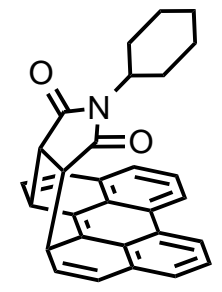<smiles>c1cc2ccc3cccc4ccc(c1)c2c34</smiles><smiles>O=C1C=CC(=O)N1C1CCCCC1</smiles><smiles>O=C1C2C(=O)N(C3CCCCC3)C(=O)C2C2C1C1C=CC(C3CCCCC3)C12</smiles>

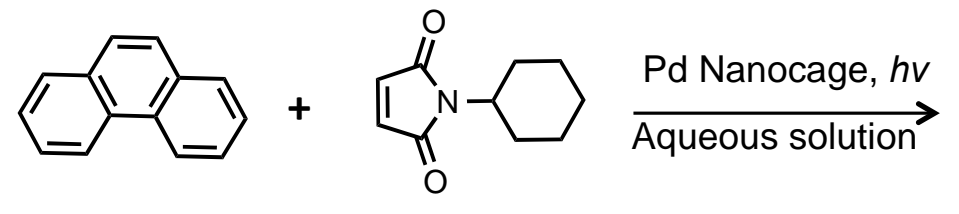<smiles>O=C1C2C(C(=O)N1C1CCCCC1)C1c3ccccc3C2C2C=CC=CC21</smiles><smiles>[R]c1ccc2c3c(cccc13)-c1ccccc1-2</smiles>

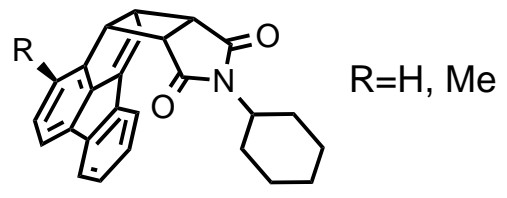

Scheme 12 Additional examples of photodimerization within Pd nanocage reported by Fujita group. 

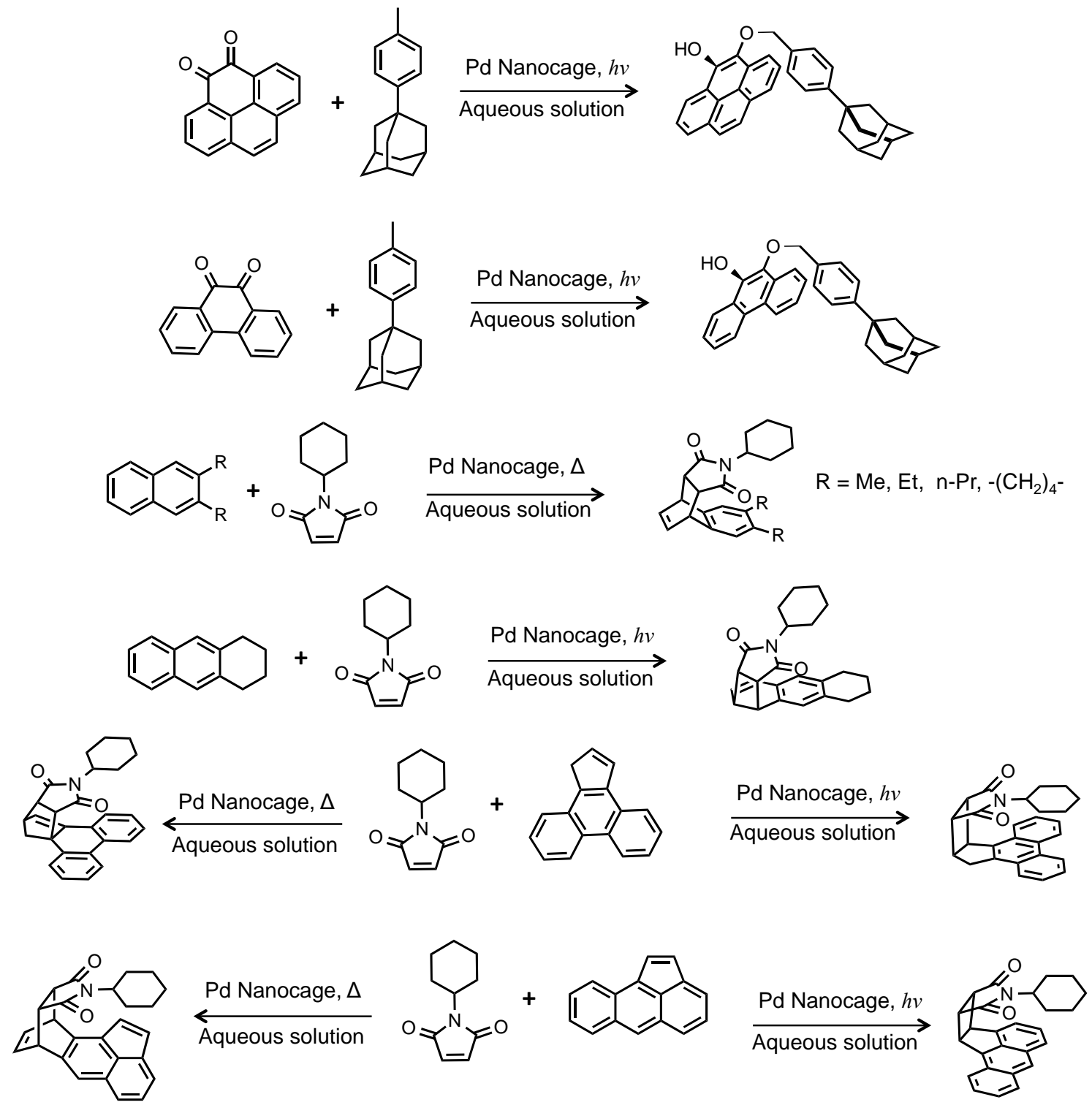

Scheme 13 Further examples of photodimerization within Pd nanocage reported by Fujita group. 


\begin{tabular}{|c|c|c|c|}
\hline $\mathrm{Ar}=\mathrm{R}-\mathrm{Ph}$ & & & \\
\hline $\mathrm{Ar}$ & & $\begin{array}{l}\text { \% Syn } \mathrm{HH} \text { dimer } \\
\text { in } \mathrm{Pd} \text { nanocage }\end{array}$ & $\%$ of cis isomer \\
\hline $\mathrm{R}=3-\mathrm{CH}_{3}$ & & 63 & 37 \\
\hline $\mathrm{R}=4-\mathrm{CH}_{3}$ & & 45 & 55 \\
\hline $\mathrm{R}=3-\mathrm{OCH}_{3}$ & & 42 & 58 \\
\hline $\mathrm{R}=4-\mathrm{OCH}_{3}$ & & 40 & 60 \\
\hline
\end{tabular}

Scheme 14 Photodimerization of cinnamic acid derivatives included within Pd nanocage. Yields are reported in relative mole percentage. 


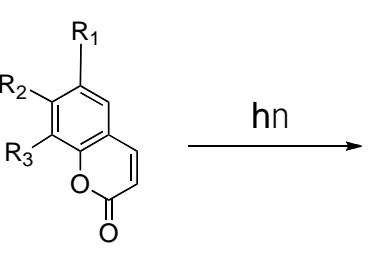

$\begin{array}{llc} & & \text { Syn HH } \\ \mathbf{R}_{\mathbf{1}}=\mathbf{R}_{\mathbf{2}}=\mathbf{R}_{\mathbf{3}}=\mathbf{H} & \text { Water } & \\ & \text { Pd-nanocage } & >90 \\ & & \\ & & 15 \\ \mathbf{R}_{\mathbf{1}}=\mathbf{M e} & \text { Water } & \\ \mathbf{R}_{\mathbf{2}}=\mathbf{R}_{\mathbf{3}}=\mathbf{H} & \text { Pd-nanocage } & \\ & & \\ \mathbf{R}_{\mathbf{2}}=\mathbf{O M e} & & \\ \mathbf{R}_{\mathbf{1}}=\mathbf{R}_{\mathbf{3}}=\mathbf{H} & \text { Water } & >90 \\ & \text { Pd-nanocage } & \\ \mathbf{R}_{\mathbf{3}}=\mathbf{O M e} & & \text { Not soluble } \\ \mathbf{R}_{\mathbf{1}}=\mathbf{R}_{\mathbf{2}}=\mathbf{H} & \text { Water } & \end{array}$
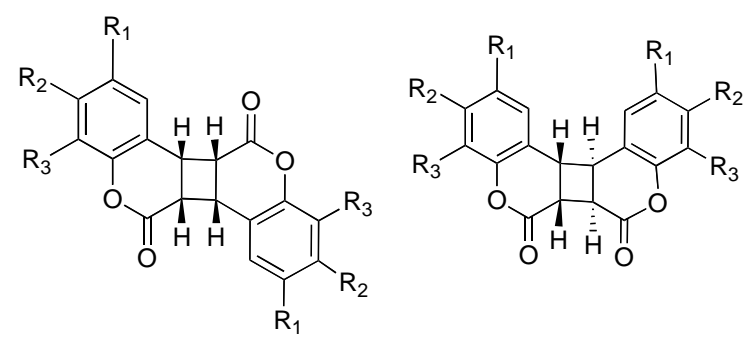

Syn HT

Anti HH

40

85

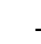

$>90$

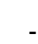

Scheme 15 Photodimerization of coumarin derivatives included within Pd nanocage. 

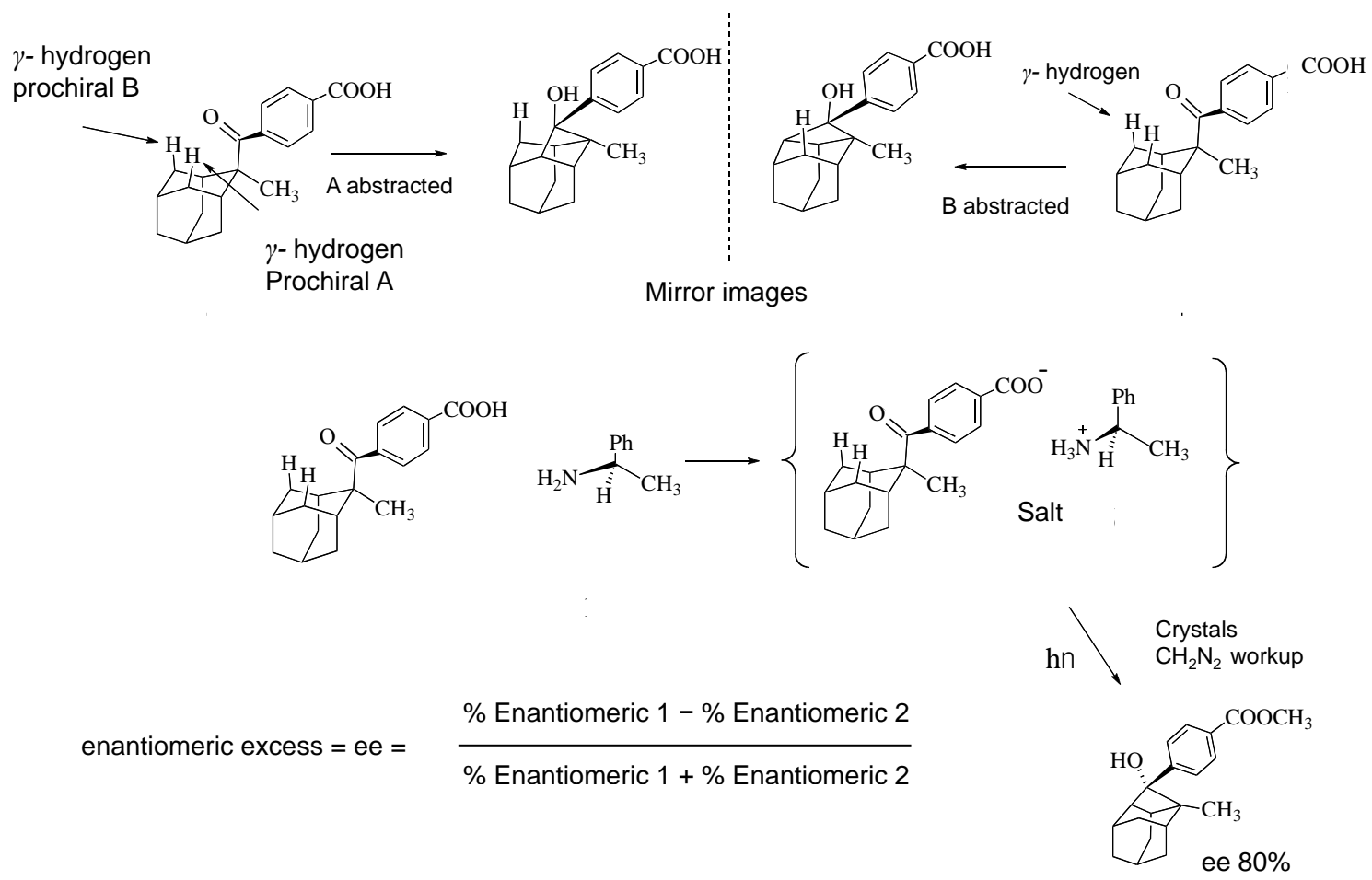

Scheme 16 Intramolecular $\gamma$-hydrogen abstraction reaction in ketones to yield enantiomeric cyclobutanols. Depending on which pro-chiral hydrogen is abstracted different enantiomer is obtained. 


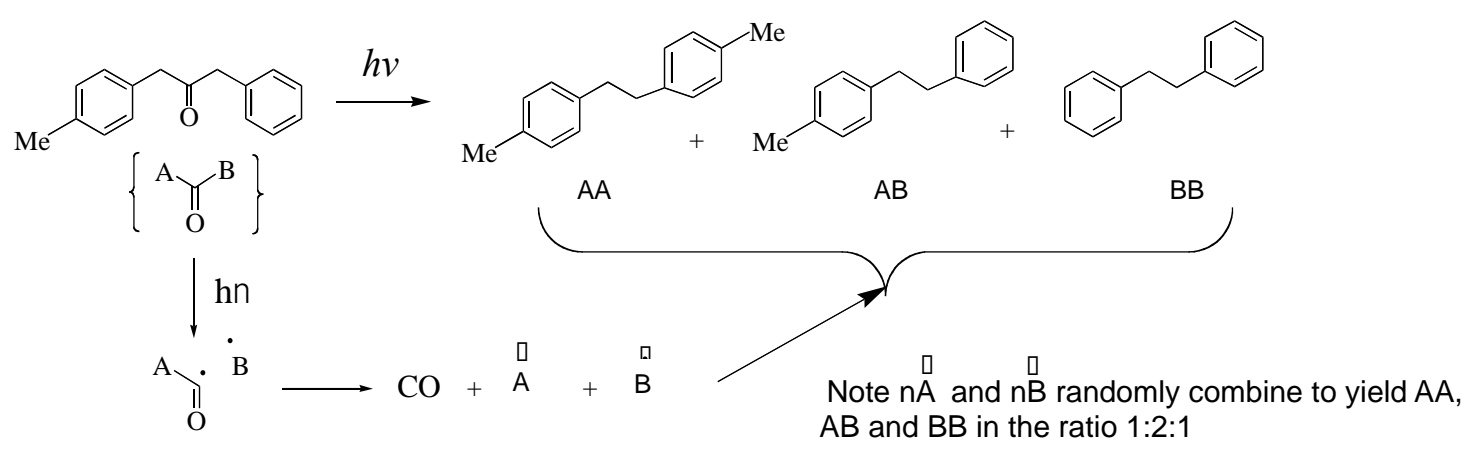

Scheme 17 Photolysis of 3-(4-methylphenyl)-1-phenylacetone (MeDBK) in isotropic solvent results in a 1:2:1 mixture of three products $\mathrm{AA}, \mathrm{BB}$, and $\mathrm{AB}$. 


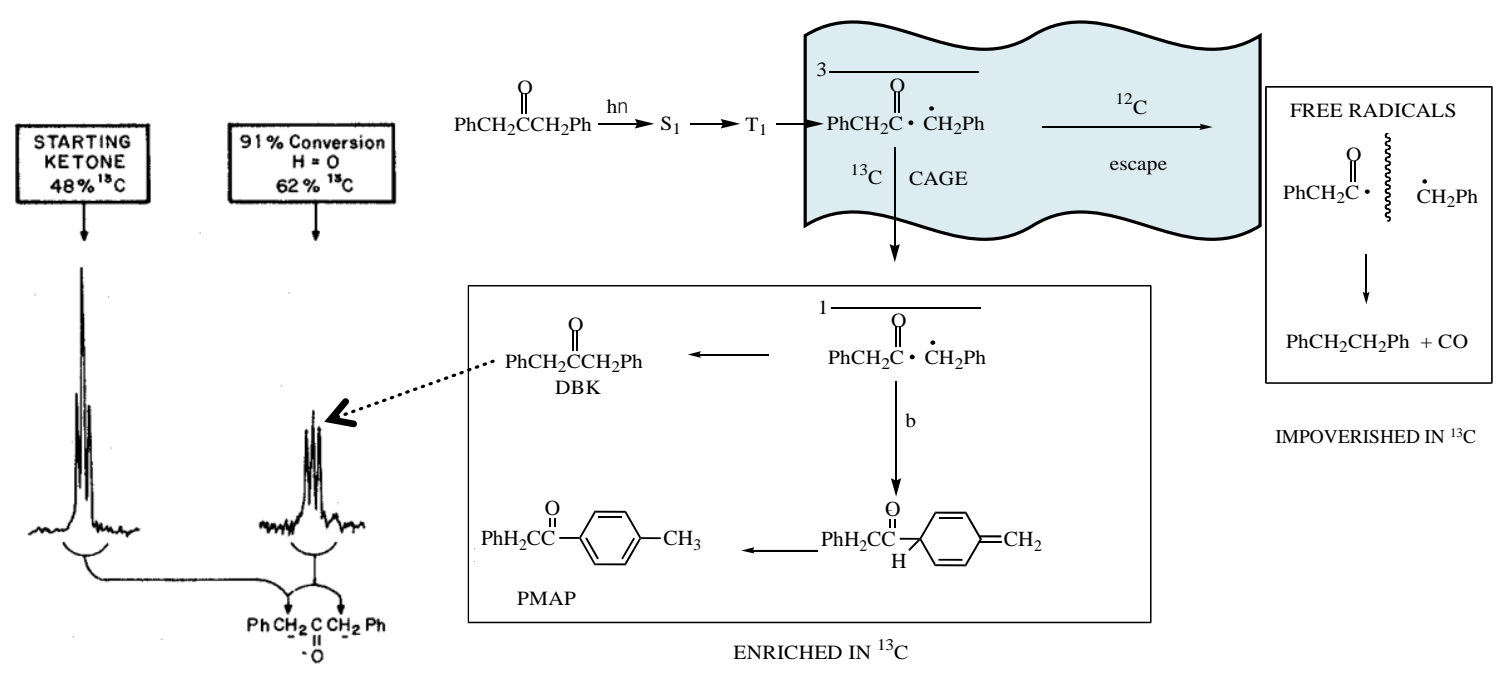

Scheme 18 Isotope enrichment observed during the photolysis of DBK due to cage effect provided by an isotropic solvent and micelle. A mixture of ${ }^{12} \mathrm{C}$ and ${ }^{13} \mathrm{C}$ enriched $(\mathrm{C}=\mathrm{O})$ DBK was irradiated in a micelle and the enrichment in the recovered DBK was followed by ${ }^{13} \mathrm{C}$ NMR. 


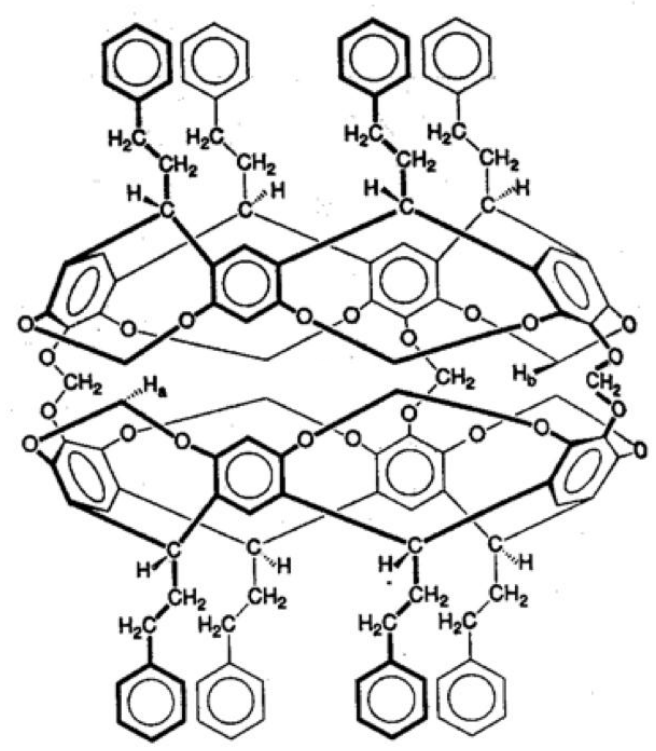

Scheme 19 Structure of a hemicarcerand used to store reactive molecules and intermediate. Note unlike cyclodextrin this host is closed at both (top and bottom) ends. 


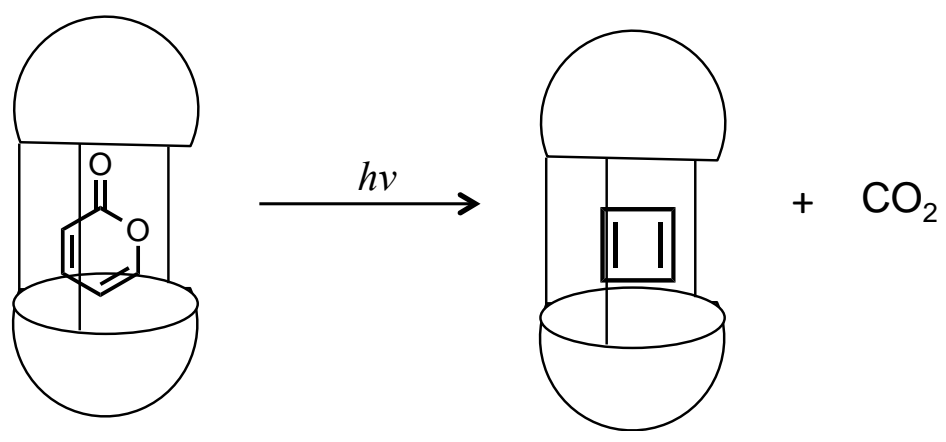

Scheme $20 \alpha$-Pyrone trapped in a hemicarcerand generates cyclobutadiene upon irradiation. 


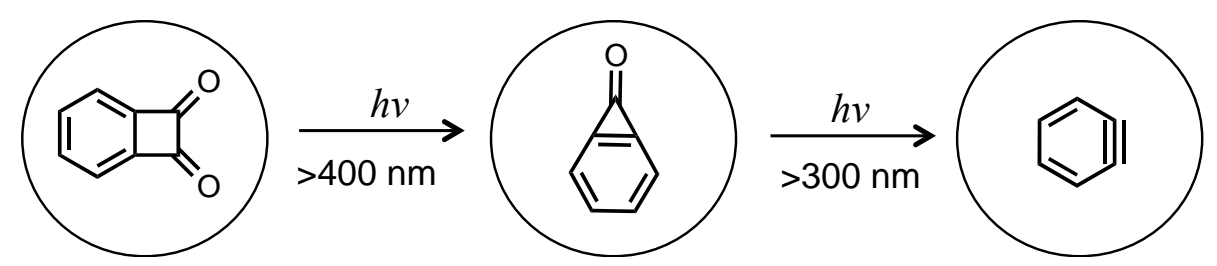

Scheme 21 Photolysis (>400 nm) of benzocyclobutenedione trapped in a hemicarcerand generates benzocyclopropanone and upon further irradiation $(>300 \mathrm{~nm})$ of benzocyclopropanone yields benzyne. 


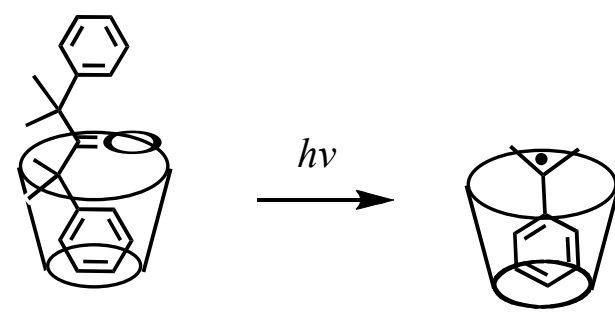

$\beta$-cyclodextrin solid

stable for days

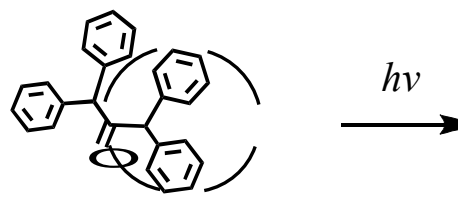

ZSM-5 zeolite

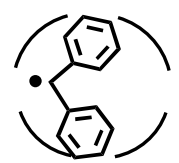

stable for weeks
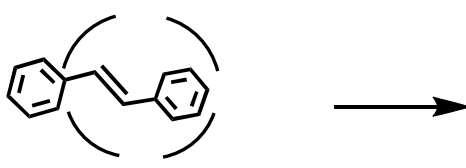

ZSM-5 zeolite

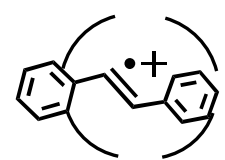

stable for months
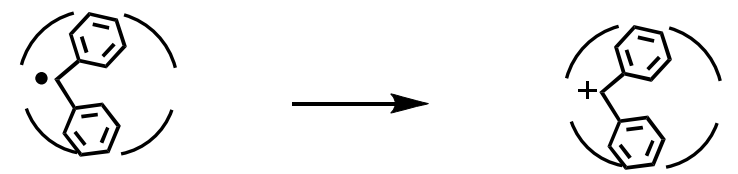

ZSM-5 zeolite

stable for weeks
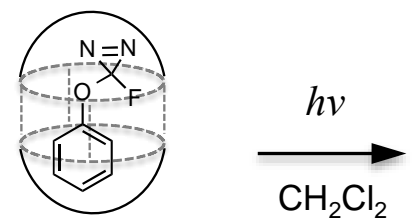

Cram's carcerand

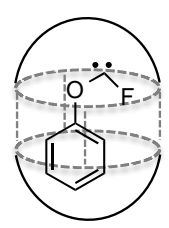

stable for days

Scheme 22 Normally short lived ( $\mu$ s) radicals, cation radicals, cations and carbenes in solution at ambient temperature upon generation within cyclodextrin, zeolite or carcerand have lifetime of days, weeks or even months. 

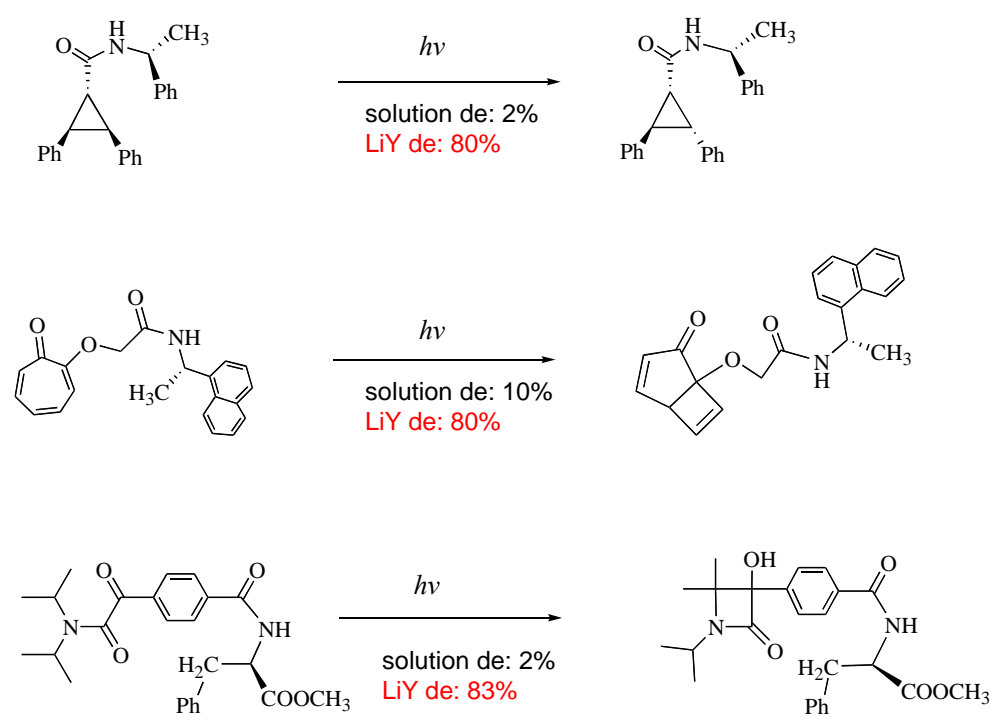

Scheme 23 Chiral induction in a photochemical reaction is achieved within an active reaction cavity of a zeolite. Interaction between the reactant and the zeolite cation plays an important role in the chiral induction. The chiral inductor is covalently linked to the reactant. 

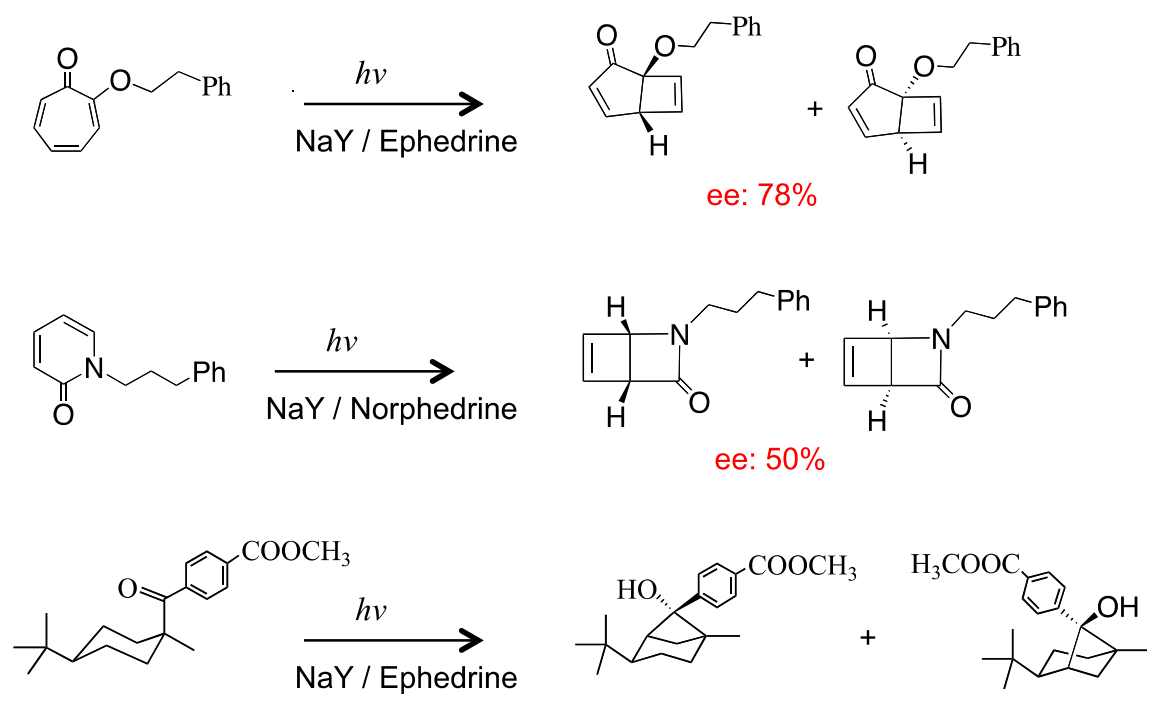

ee: $30 \%$

In solution the same chiral indicator has no effect

Scheme 24 Chiral induction in a photochemical reaction is achieved within an active reaction cavity of a zeolite. Interaction between the reactant and the zeolite cation plays an important role in the chiral induction. The chiral inductor is present as an independent molecule. 


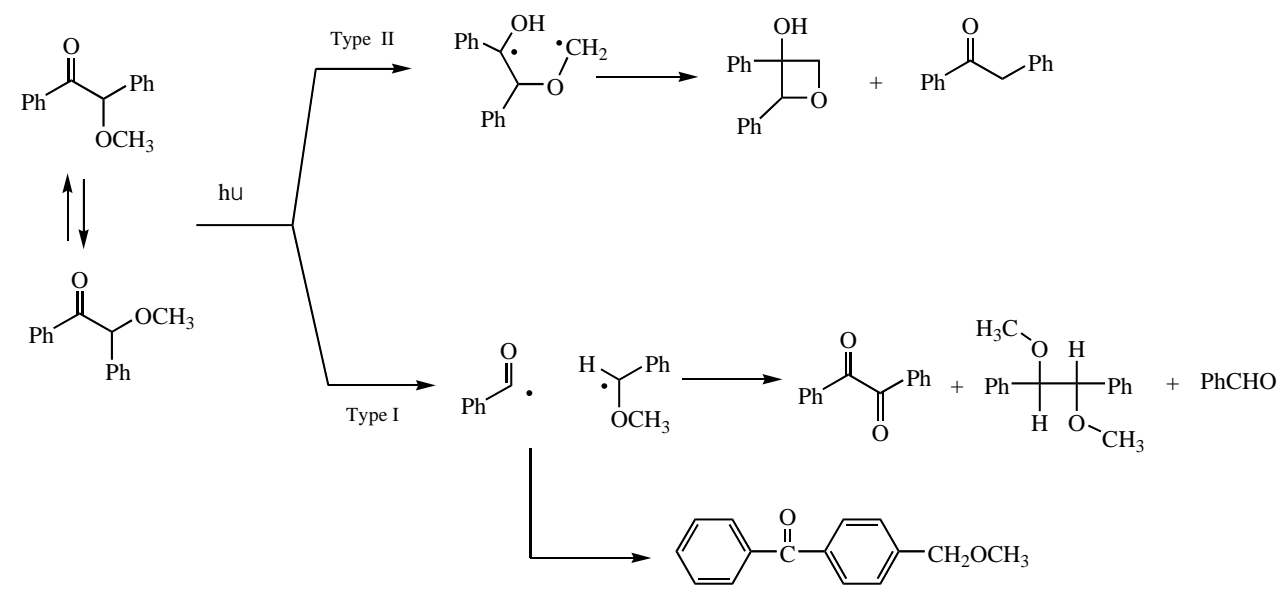

Scheme 25 Type I and Type II photoreaction of benzoin alkyl ethers. 


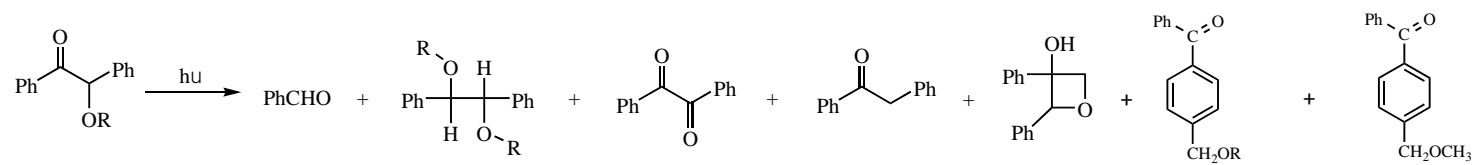

\begin{tabular}{|c|c|c|c|c|c|c|c|}
\hline \multicolumn{8}{|l|}{$\mathbf{R}=\mathbf{C H}_{\mathbf{3}}$} \\
\hline Benzene & $27 \%$ & $39 \%$ & $23 \%$ & -- & -- & -- & -- \\
\hline $\begin{array}{l}\text { Cetyl trimethyl ammonium } \\
\text { chloride micelle }\end{array}$ & $14 \%$ & $8 \%$ & $7 \%$ & $4 \%$ & $52 \%$ & -- & -- \\
\hline \multicolumn{8}{|l|}{$\mathrm{R}=\mathrm{CH}_{2}-\left(\mathrm{CH}_{2}\right)_{6}-\mathrm{CH}_{3}$} \\
\hline Benzene & $12 \%$ & $49 \%$ & -- & $3 \%$ & -- & -- & -- \\
\hline $\begin{array}{l}\text { Cetyl trimethyl ammonium } \\
\text { chloride micelle }\end{array}$ & $3 \%$ & $36 \%$ & -- & $2 \%$ & -- & $45 \%$ & $45 \%$ \\
\hline
\end{tabular}

Scheme 26 Product distribution upon irradiation of benzoin methyl ether and benzoin octyl ether in benzene and CTAB micelle. 
(a)
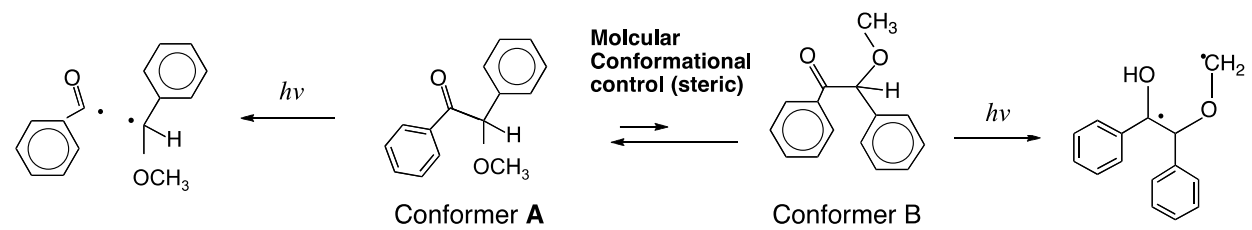

I(RP)

${ }^{*} \mathrm{R}(\mathrm{A})$

*R(B)

$\mathrm{I}(\mathrm{BR})$

(b)

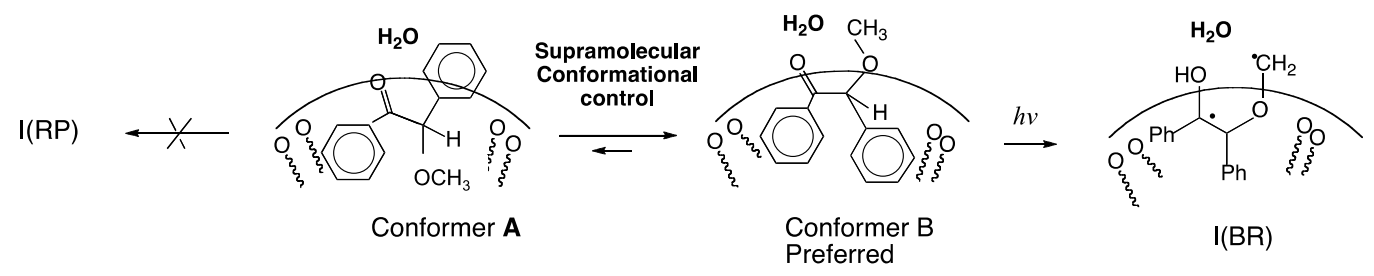

(c)
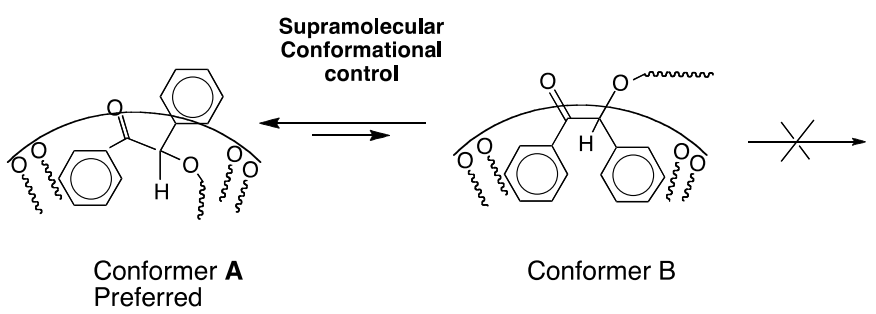

I(BR)

I(RP)

Preferred

Conformer B

Scheme 27 Preferred orientation of benzoin methyl ether and benzoin octyl ether at micelle-water interface. Benzoin alkyl ether can exist in two conformations; depending on the chain length only one is preferred at the interface. 


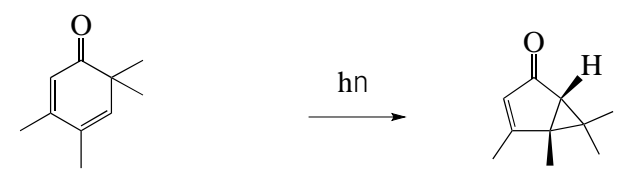

$\mathrm{NaY/(+)ephedrine:} \mathrm{ee}$

$30 \%$ at $23^{\circ} \mathrm{C}$;

$49 \%$ at $-55^{\circ} \mathrm{C}$
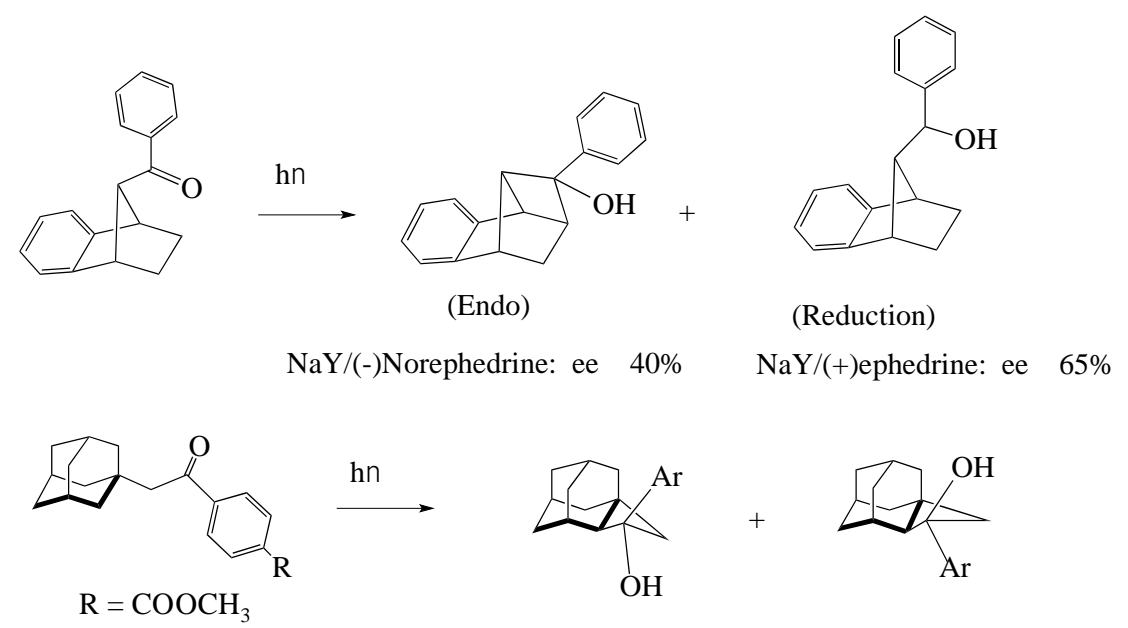

$\mathrm{NaY/(+)ephedrine:} \mathrm{ee}$

$35 \%$

$\sim 5 \%$
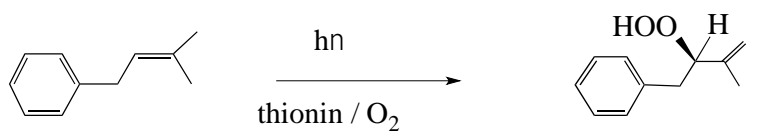

$\mathrm{NaY} /(+)$ ephedrine. $\mathrm{HCl}$ : ee $16 \%$

Scheme 28 Selected examples of asymmetric photoreactions in zeolites. Chiral induction is brought forth by co-inclusion of a chiral inductor with reactant molecules. 

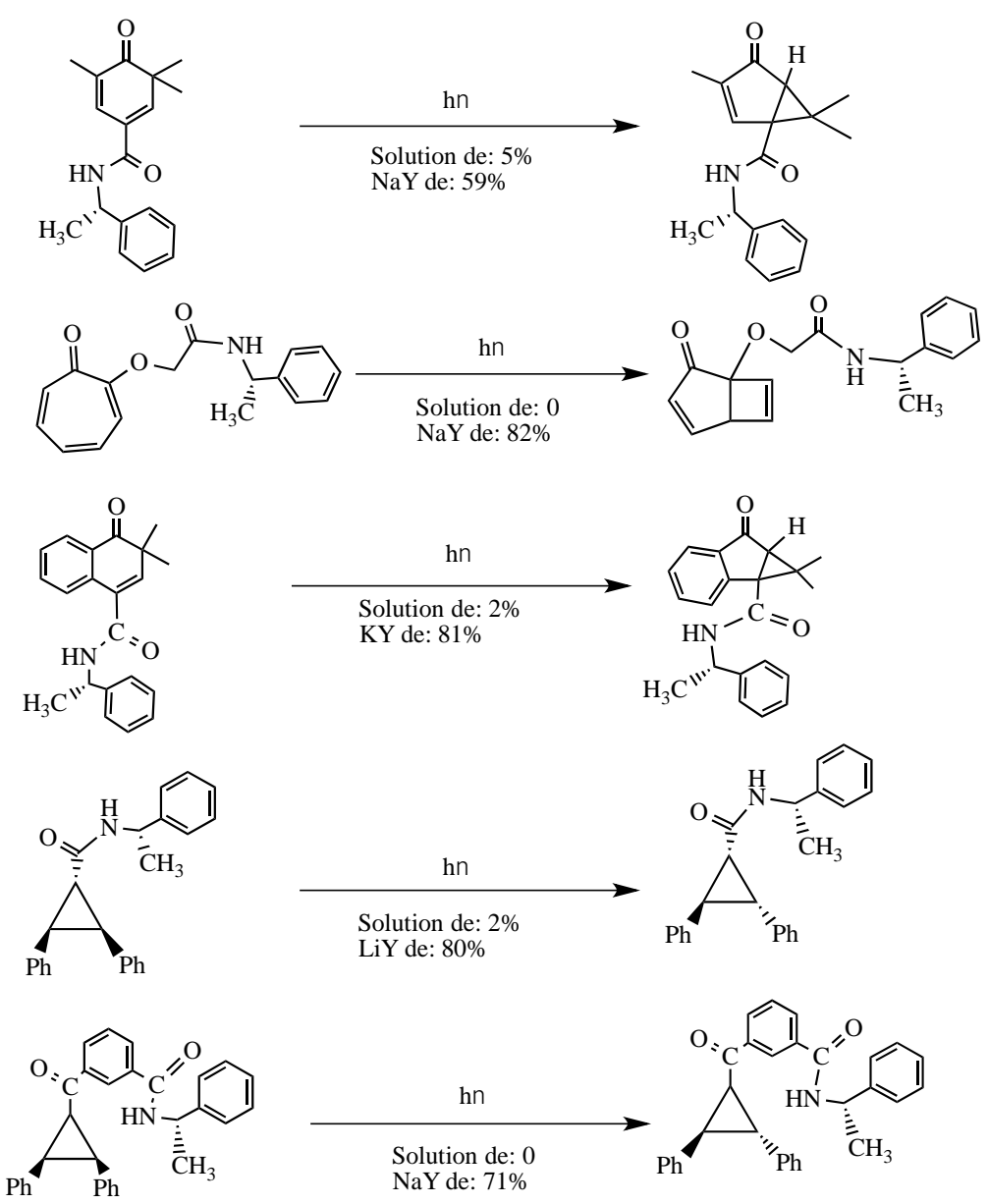

Scheme 29. Selected examples of asymmetric photoreactions in zeolites. Chiral induction is brought forth by chiral auxiliary covalently linked to the reactant molecules. Enhanced diastereomeric excess is obtained within a zeolite. 

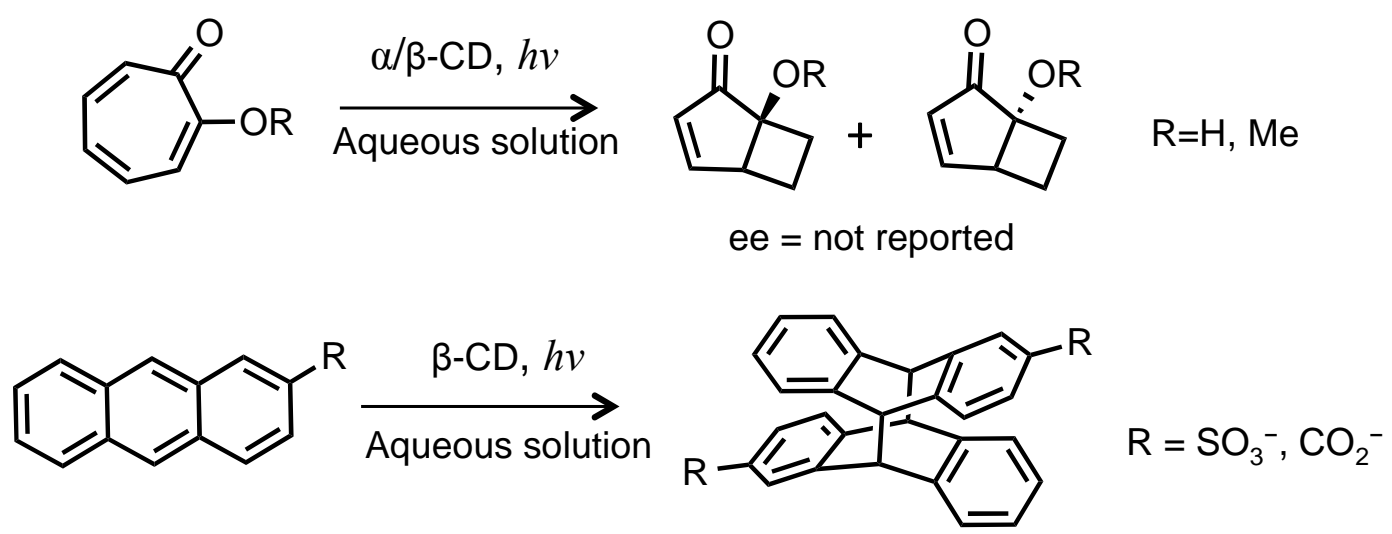

ee $=$ not reported<smiles>O=Cc1ccccc1</smiles><smiles></smiles>
$e e=15 \%$
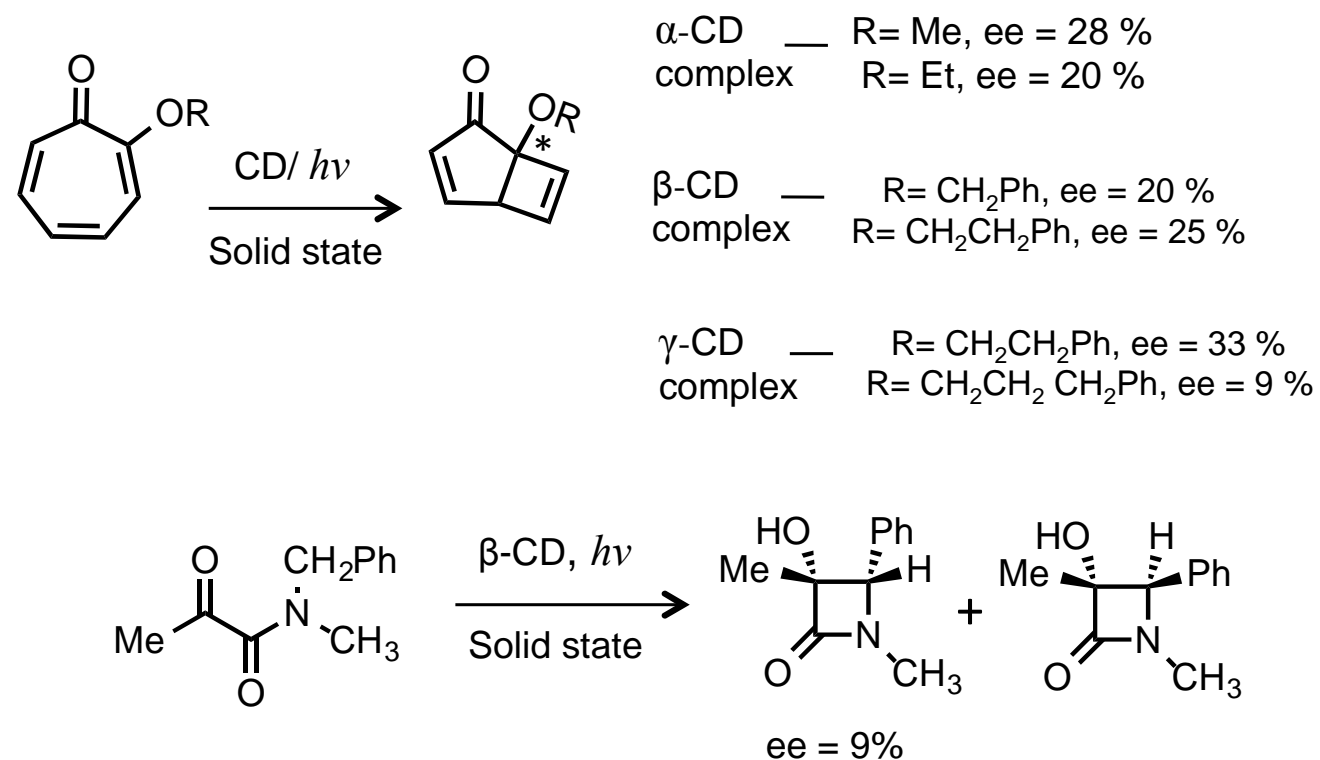

Scheme 30 Selected examples of cyclodextrins used as chiral inductor for photochemical reactions. 


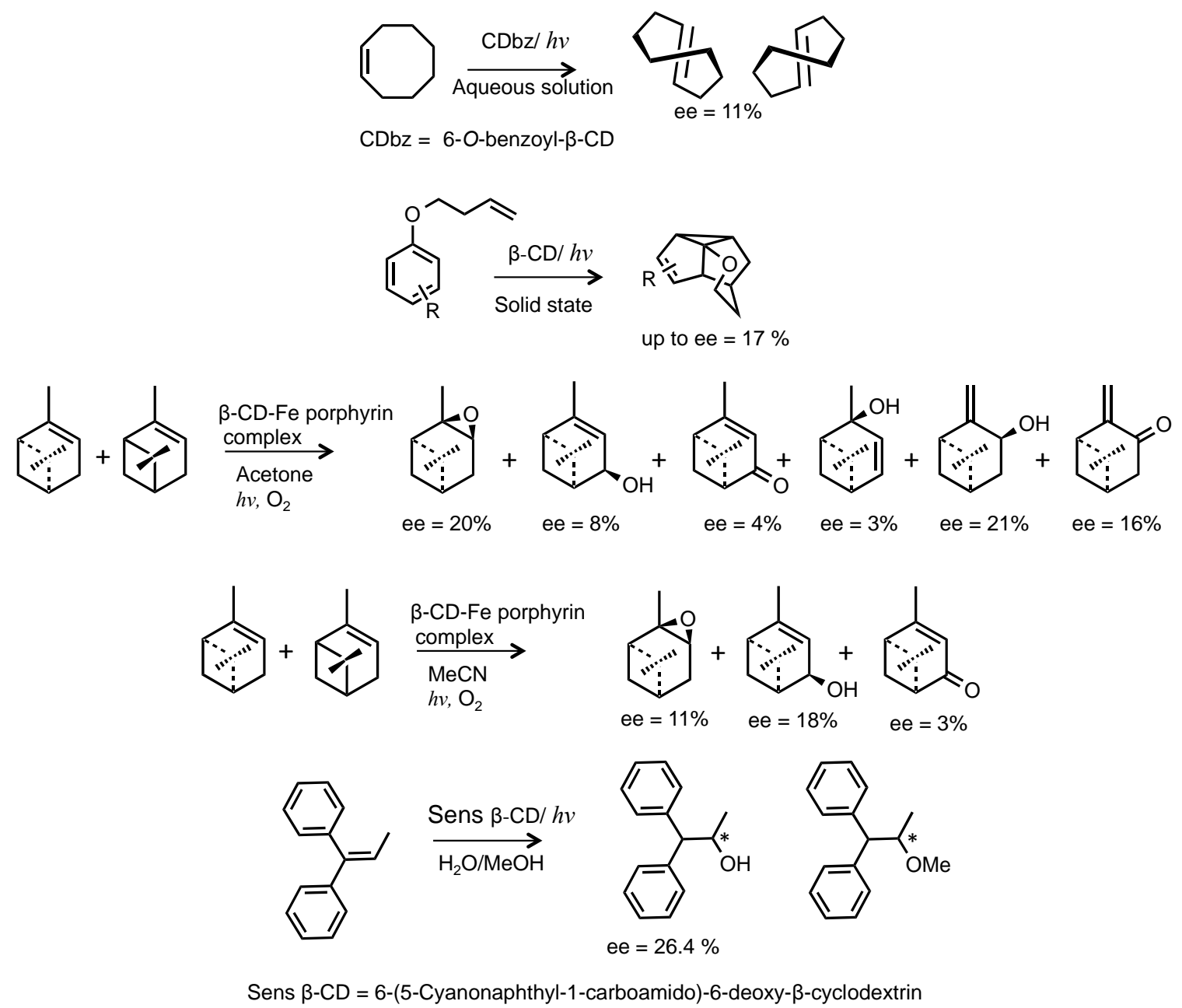

Scheme 31 Selected examples of cyclodextrins used as chiral inductor for photochemical reactions. 

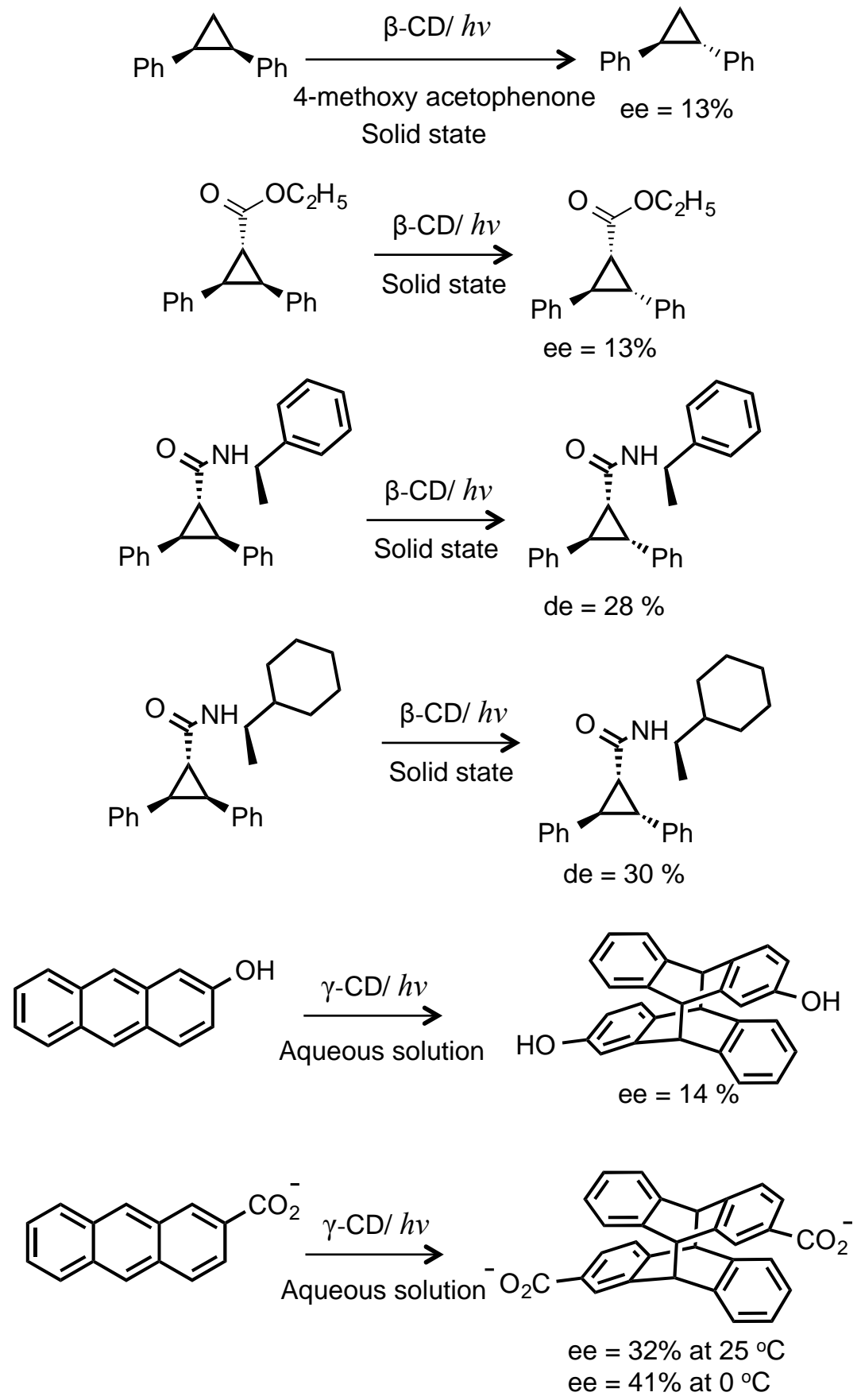

Scheme 32 Selected examples of cyclodextrins used as chiral inductor for photochemical reactions. 

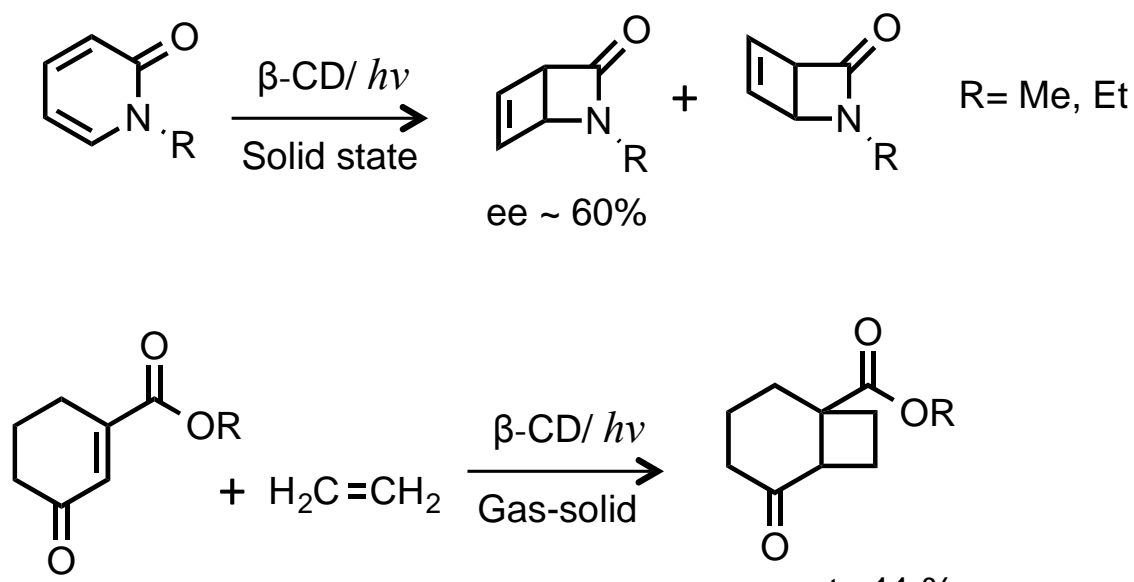

ee up to $11 \%$

OR $+\mathrm{H}_{2} \mathrm{C}=\mathrm{CH}_{2}$<smiles>[R]OC(=O)CCC(=O)O</smiles><smiles>O=C1CCC2(C(=O)O)CCC12</smiles>

ee up to $8 \%$<smiles>COC(=O)c1cc2ccccc2cc1OC</smiles>

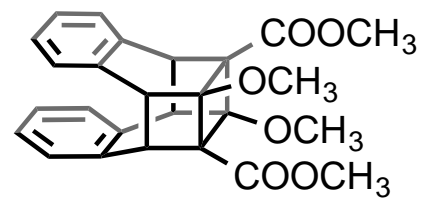

$$
\text { ee }=48 \%
$$<smiles>O=C([OH2+])c1ccc2ccccc2c1</smiles><smiles>O=C([OH2+])C12C3c4ccccc4C(c4ccccc43)C1(C(=O)[OH2+])C2CCCO</smiles><smiles>COC(=O)[C@H](C)C[P+]</smiles>

ee $=94 \%$
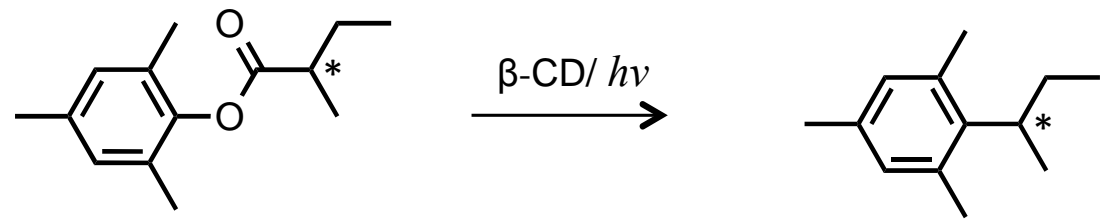

(R) $\mathrm{ee}=14 \%$

Scheme 33 Selected examples of cyclodextrins used as chiral inductor for photochemical reactions. 


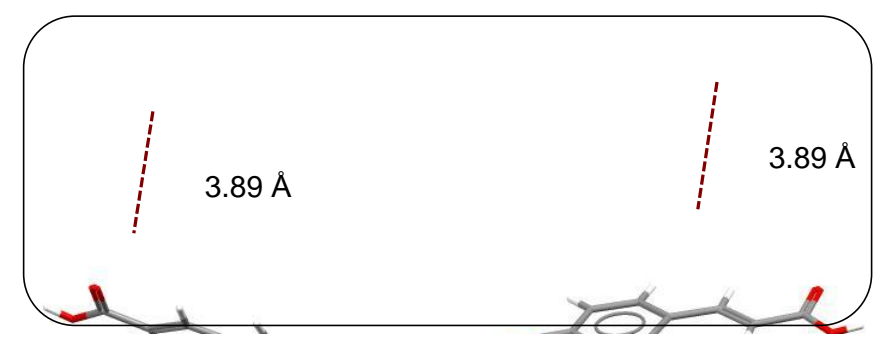

Figure $1 \beta$-Type packing arrangement of trans 4-bromo cinnamic acid. Note the two molecules are related by mirror symmetry and the reactive double bonds are within $4 \AA$ and parallel to each other. 


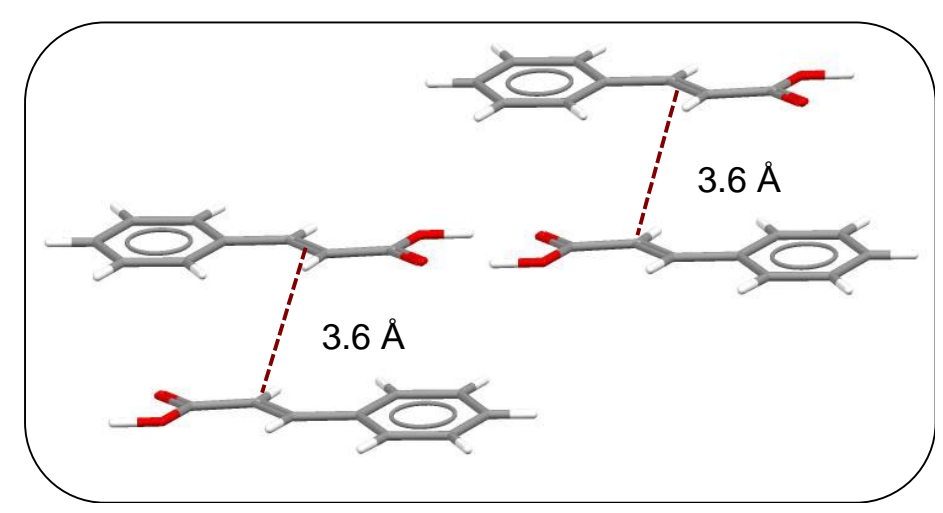

Figure $2 \alpha$-Type packing arrangement of trans cinnamic acid in crystals. Note the two molecules are related by centrosymmetry and the reactive double bonds are within $4 \AA$ and parallel to each other. 

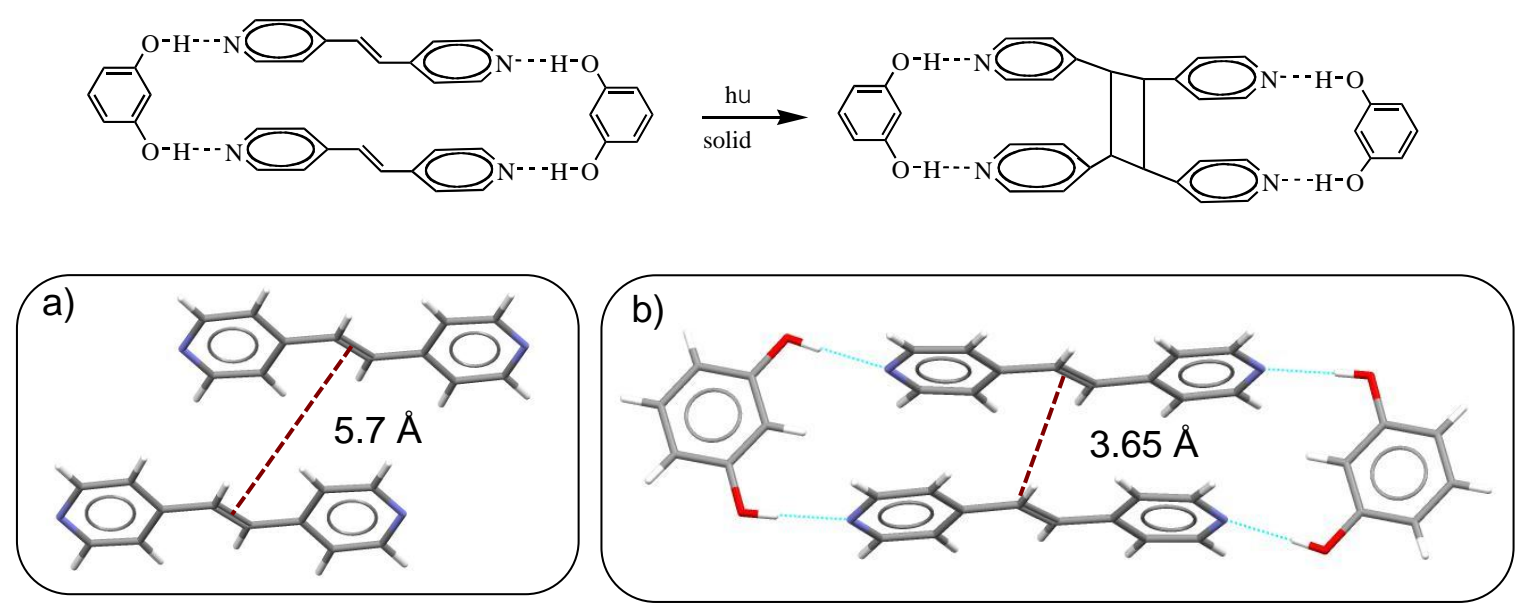

Figure 3 Top: Irradiation of a mixture of 1,3-dihydroxybenzene and trans-1, 2 bis (4pyridyl)ethylene in crystalline state results in a single photodimer.

Bottom: Crystal packing of (a) trans-1, 2 bis (4-pyridyl)ethylene and (b) co-crystal of 1,3-dihydroxybenxene with trans-1, 2 bis (4-pyridyl)ethylene. Note: In the absence of the templating agent 1,3-dihydroxybenzene the reactive double bonds are separated by $\sim 5.7 \AA$. 


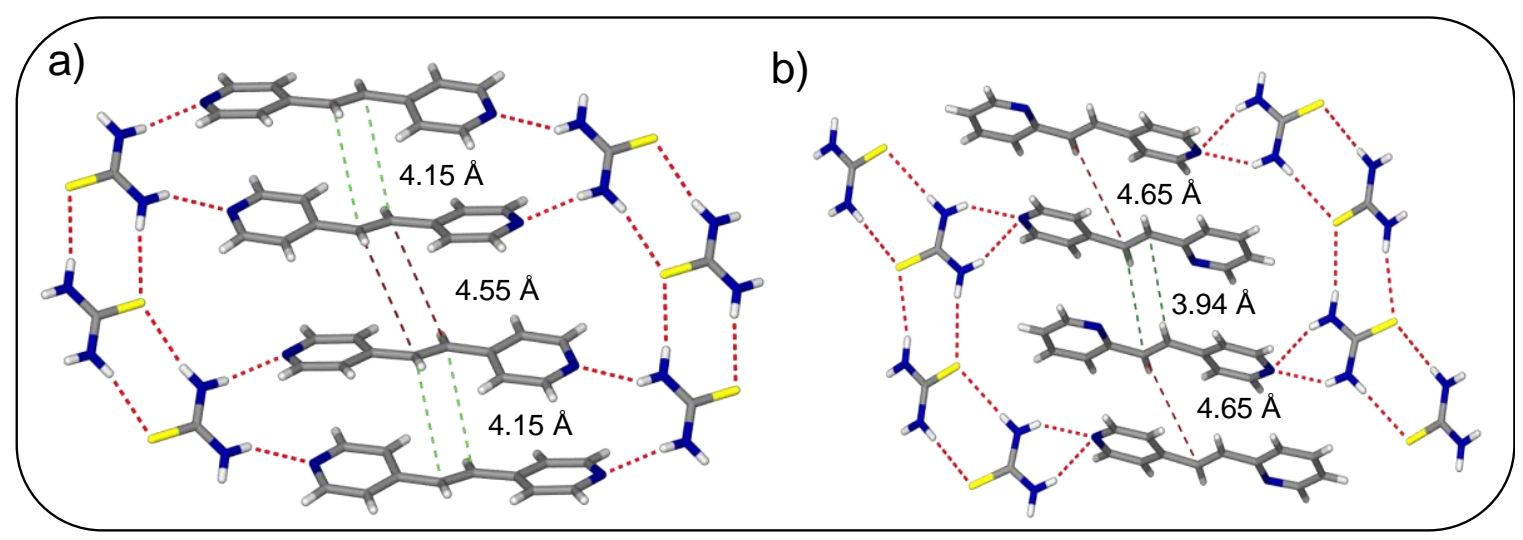

Figure 4 Crystal packing of (a) 1:1 co-crystal of thiourea with trans-1, 2 bis (4pyridyl)ethylene and (b) 2:1 co-crystal of thiourea with trans-1-(2-pyridyl)-2-(4pyridyl)ethylene. 


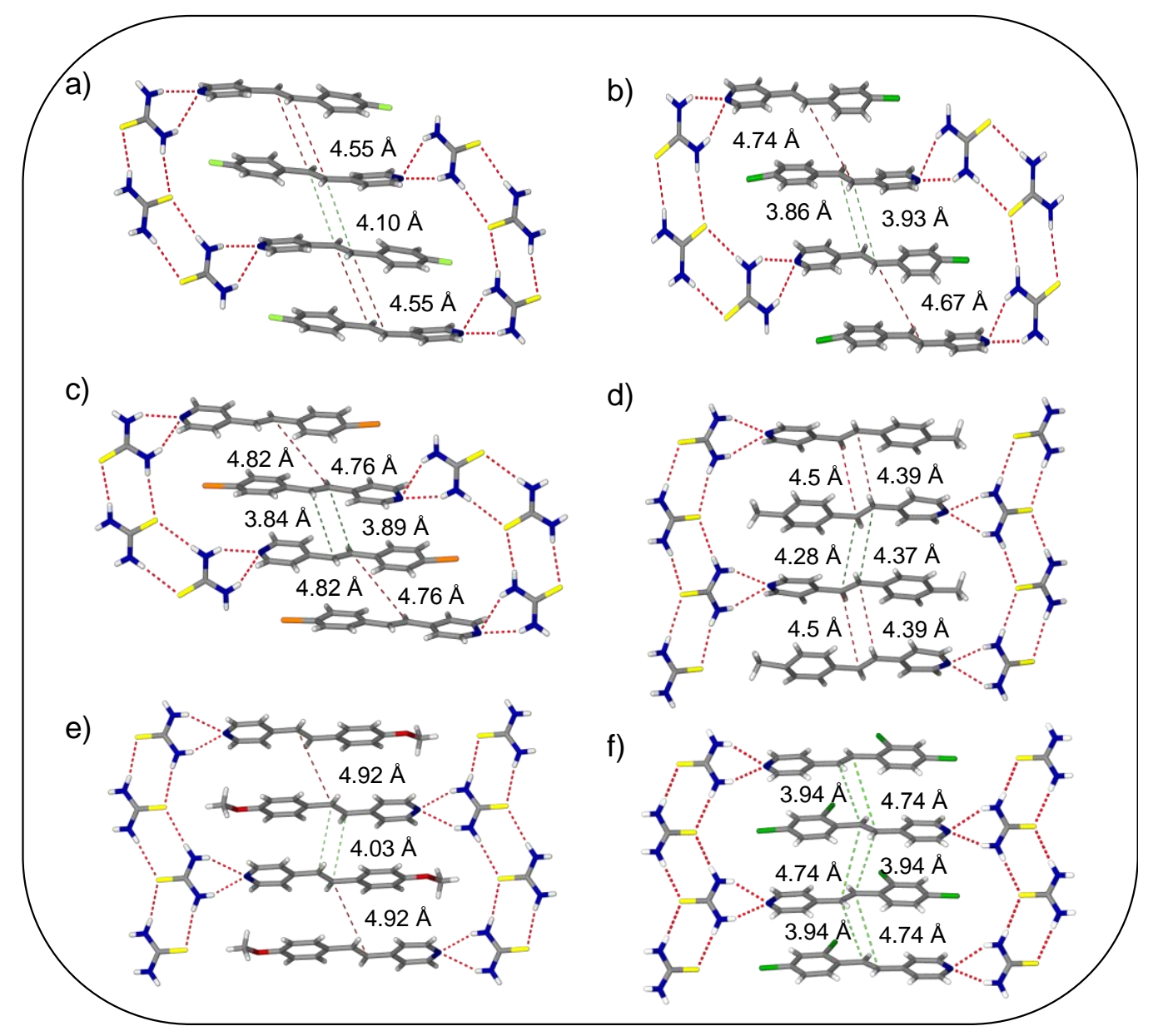

Figure 5 Crystal packing of co-crystals of thiourea with (a) trans-4-fluoro stilbazole, (b) trans-4-chloro stilbazole (c) trans-4-bromo stilbazole, (d) trans-4-methyl stilbazole, (e) trans-4-methoxy stilbazole, and (f) trans-2,4-dichloro stilbazole 


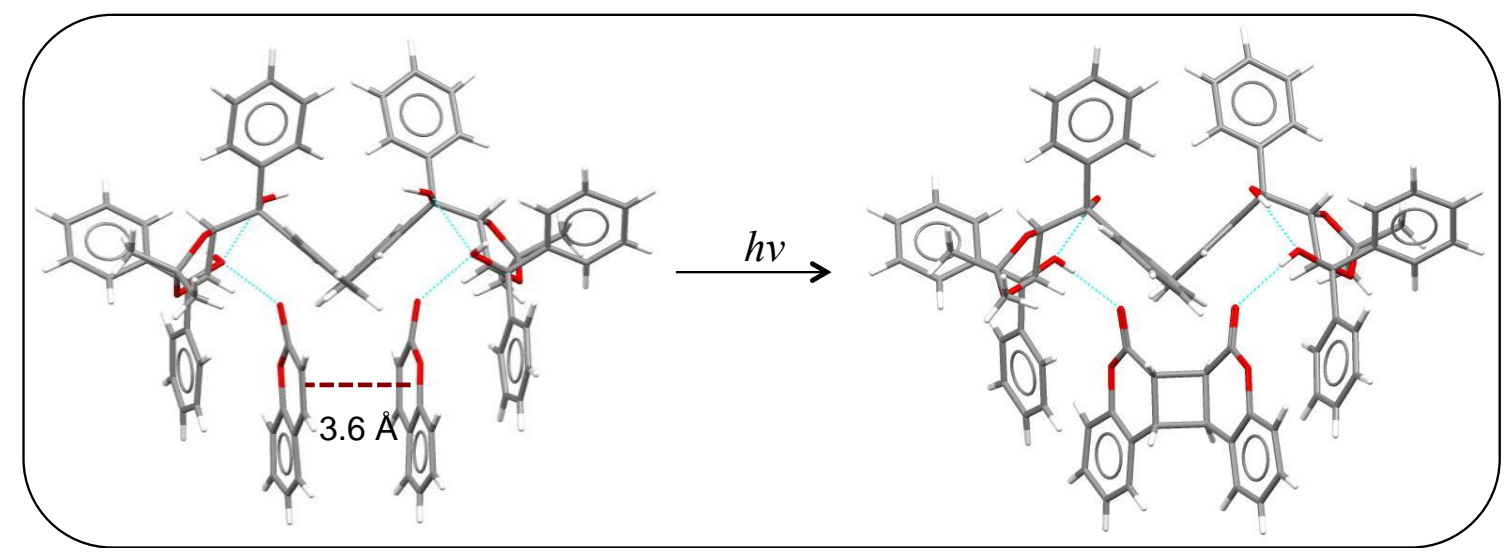

Figure 6 Packing arrangement of coumarin and the host shown in Scheme 5. Coumarin is oriented within reactive distance by optically active host and yields a single optically active dimer upon irradiation. 

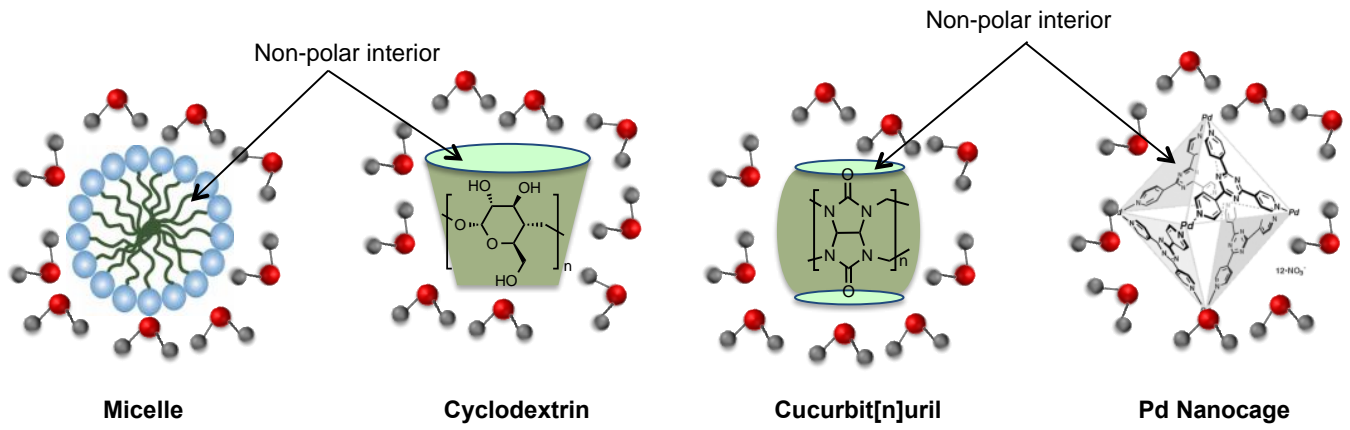

Figure 7 Cartoon representation of structures of micelle, cyclodextrin, cucurbituril and Pd nonocage in water. In these structures water molecules surround the hosts. 


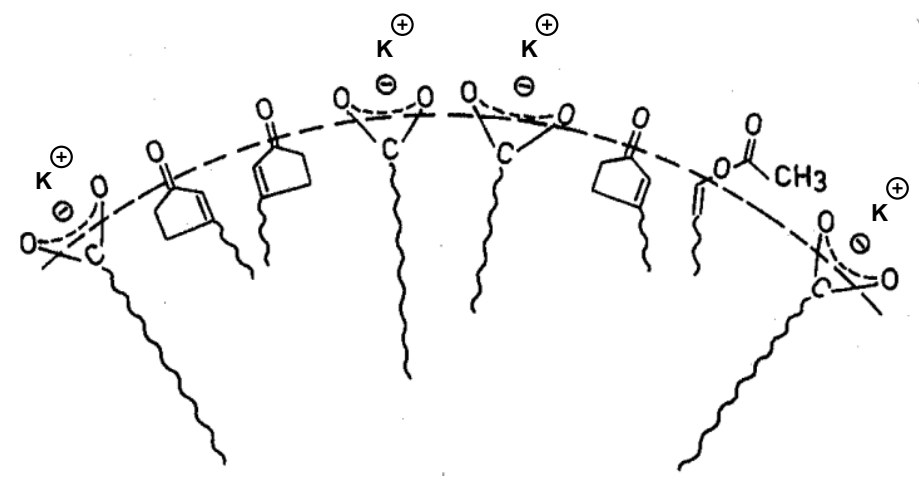

Figure 8 Orientation of 3- $n$-butylcyclopentanone and heptenyl acetate at the micellarwater interface. Polar head group face water while non-polar alkyl chains stay within the micellar core. 


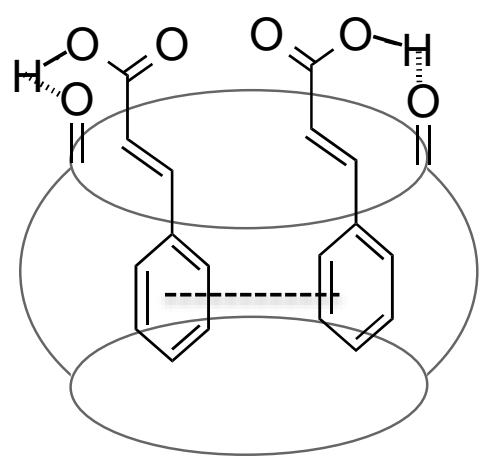

$\pi-\pi$ interaction

Figure 9 Head-head alignment of cinnamic acids in cucurbit[8] uril facilitated by $\pi-\pi$, and hydrogen bonding. 

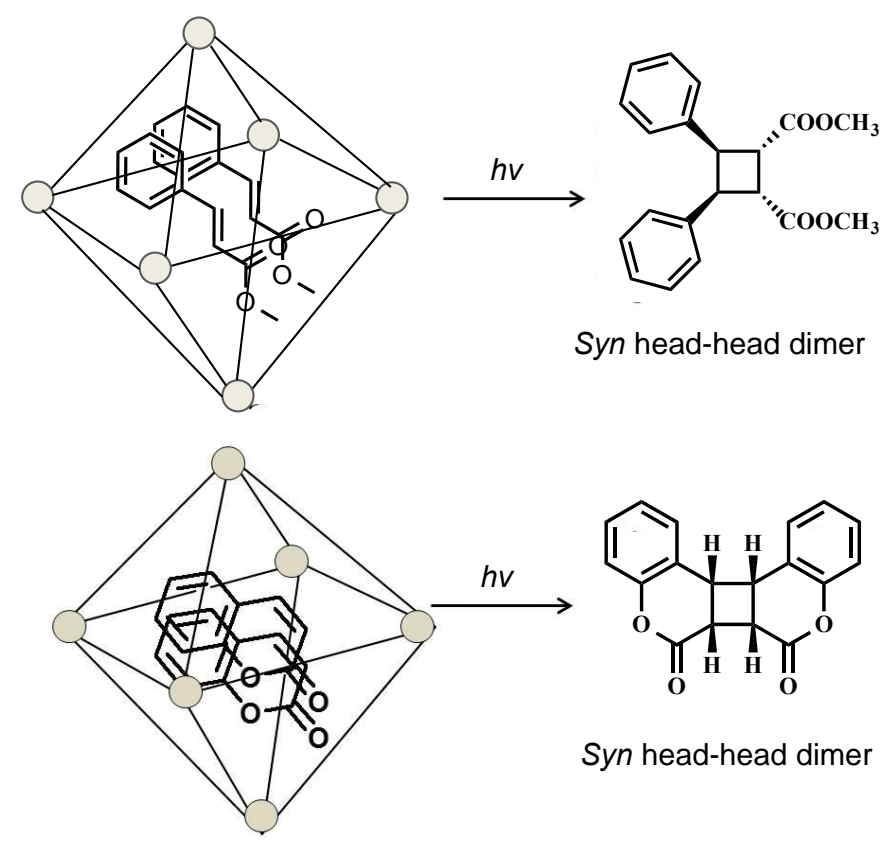

Syn head-head dimer

Figure 10. Cartoon representation of alignment of cinnamic acid and coumarin derivatives included in Pd nanocage. Cage-water interface is believed to facilitate the orientation presented in the figure. 


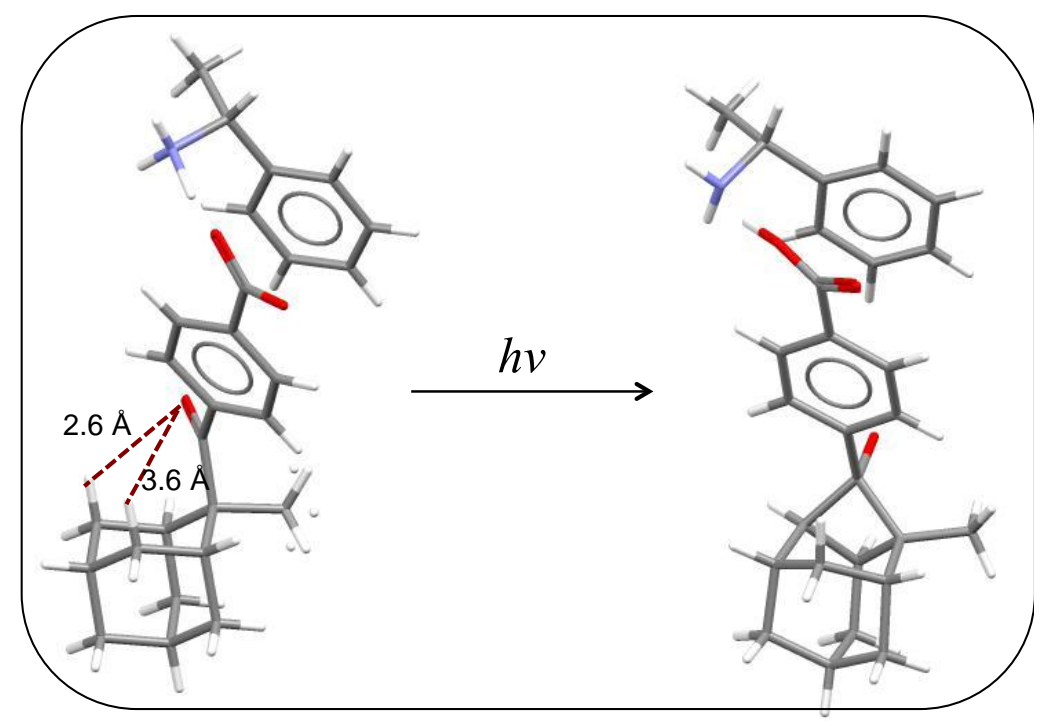

Figure 11 X-ray crystal structure of ketone-carboxylic acid-chiral amine salt shows that one prochiral hydrogen is closer than the other to the carbonyl chromophore. 


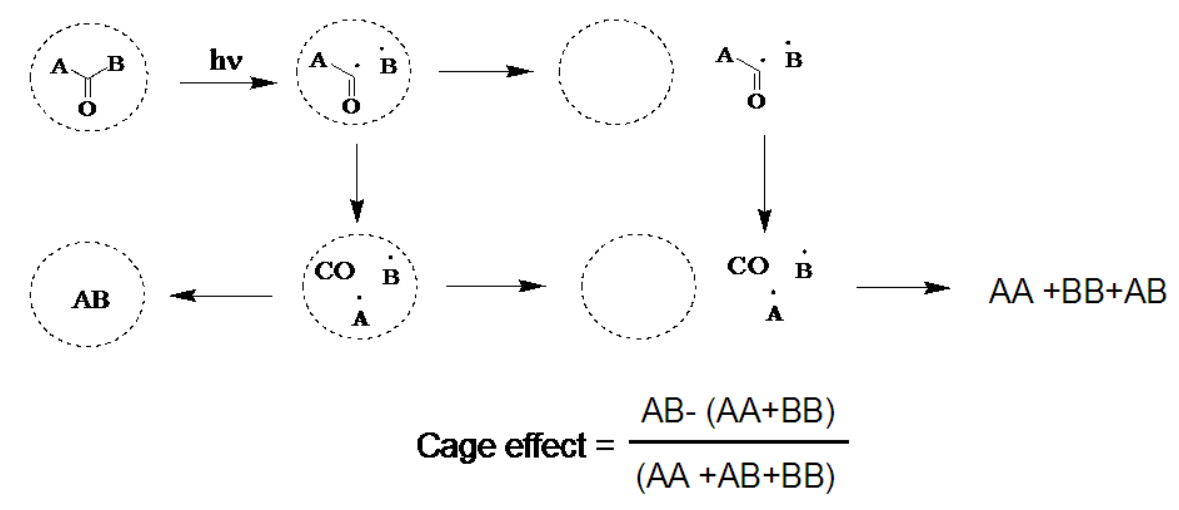

Figure 12 Photolysis of DBK like molecules in a confined space. Cage effect defined. 


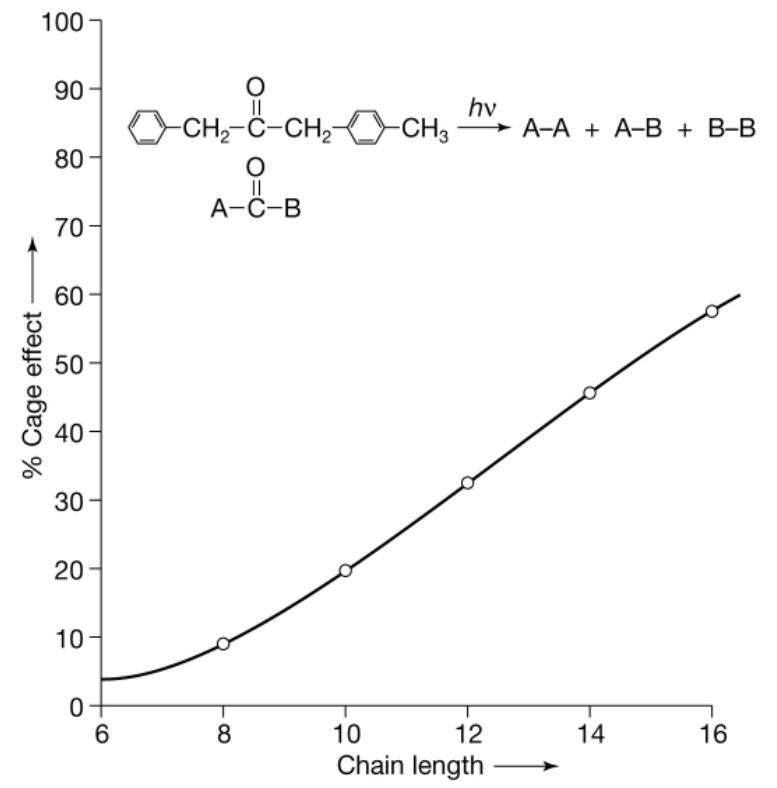

Figure 13 A plot of $\%$ cage effect vs the chain length of the alkanoic acid salt that forms the micelle. The cage effect depends on the size of the micelle. 


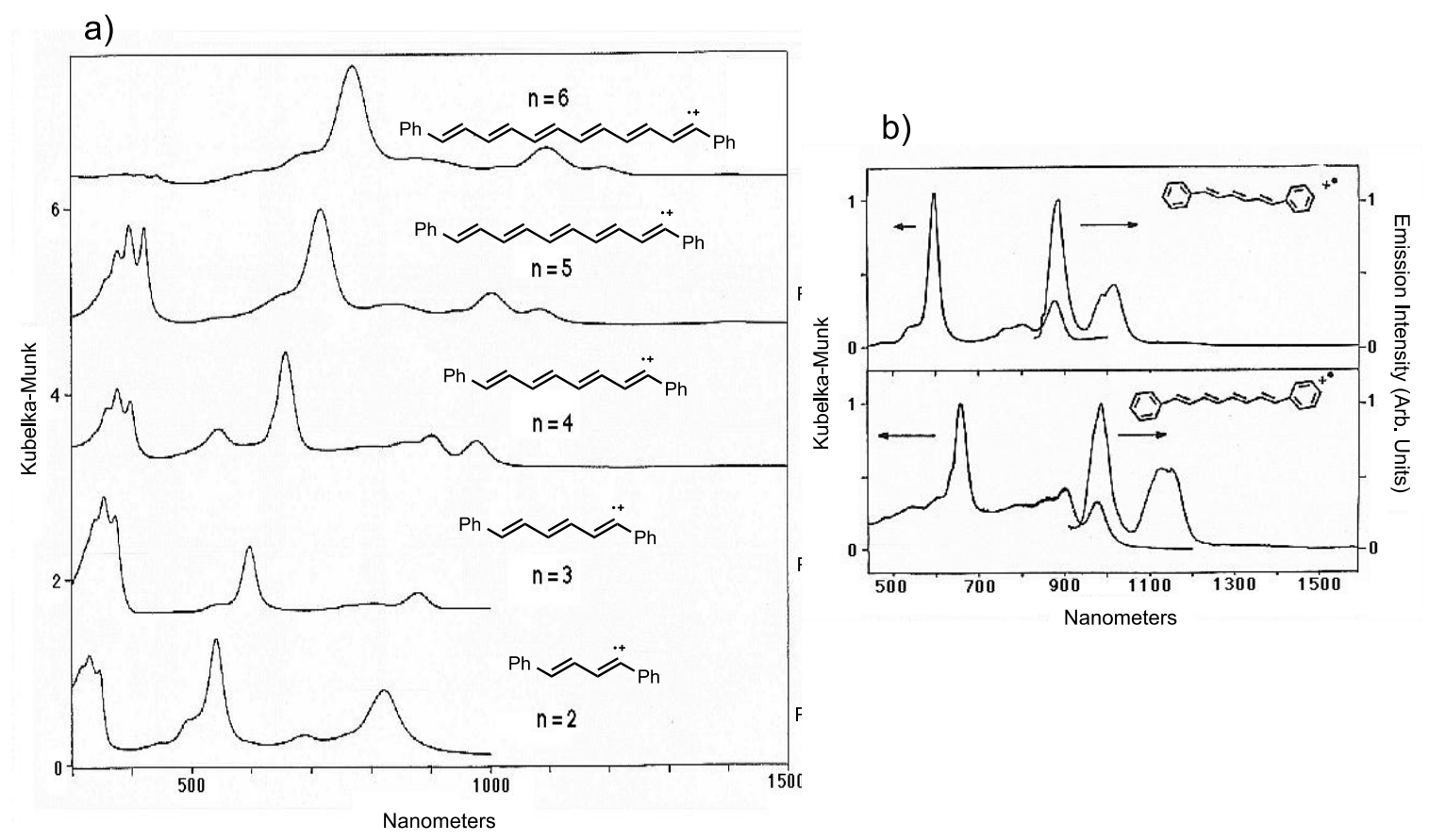

Figure 14 1,n-Diphenyl alkene cation radicals, spontaneously generated in the channels of ZSM-5 have a long lifetime. Absorption and emission spectra could be recoded under ambient condition. (a) Absorption and (b) excitation and emission spectra of a few radical cations trapped in ZSM-5 zeolites are presented. 


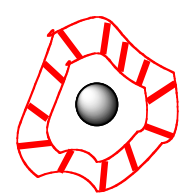

$\mathrm{R}$

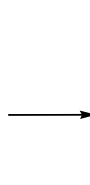

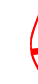

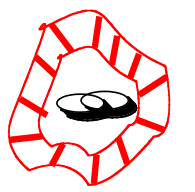

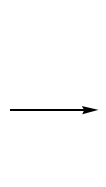

$\mathrm{P}$

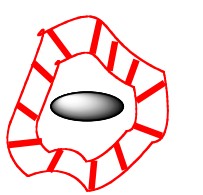

$P 1$

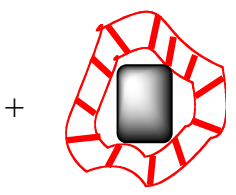

P2

Figure 15 Cartoon representation of a chemical reaction that proceeds in a series of steps (reactant-intermediate-product). Note: two products formed have different size and shape. The surrounding reaction cavity is represented in red and it does not change with the reaction. 


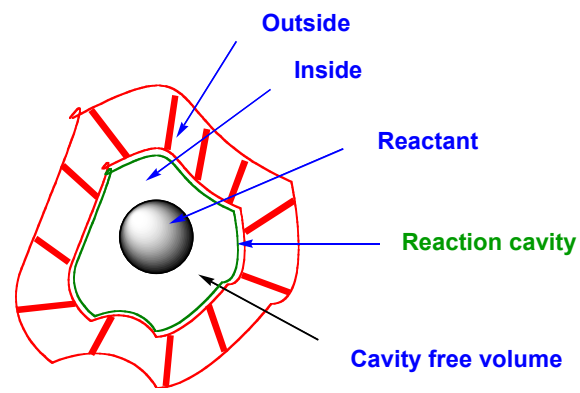

Figure 16 Cartoon representation of reaction cavity showing reactant, cavity free volume (void space surrounding reactant). 


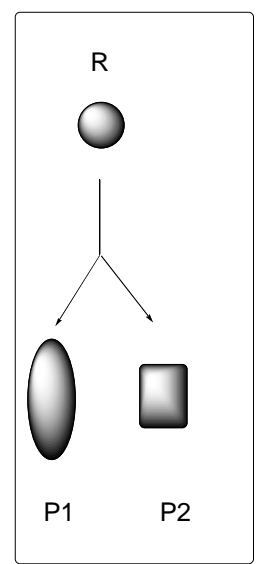

Gas phase Shape change would have no problem

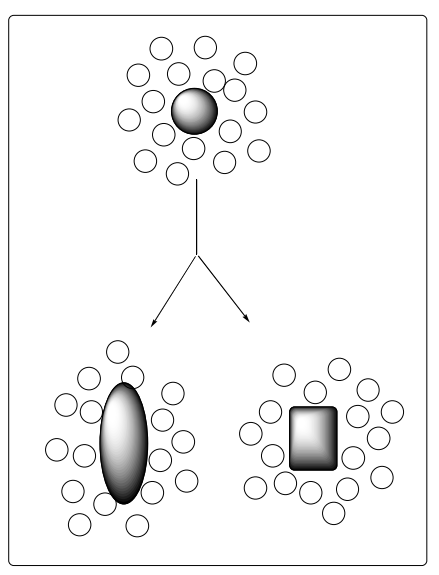

Solution phase

Fast solvent

relaxation would

allow both products

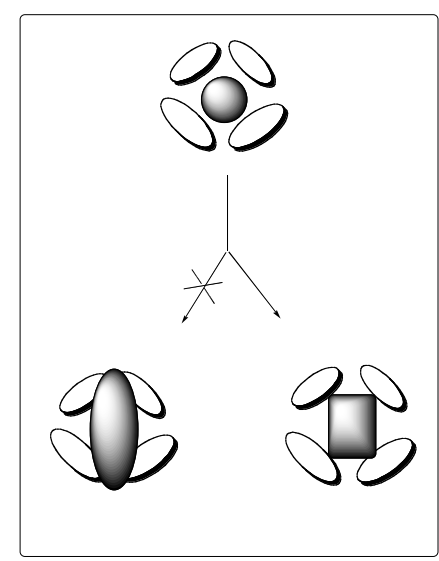

Solid phase

Rigid surroundings

permit only certain shape changes

Figure 17 Cartoon representation of influence of media on conversion of reactant to possible products. In organized media, surrounding favors one product (square) over the other (oval). 


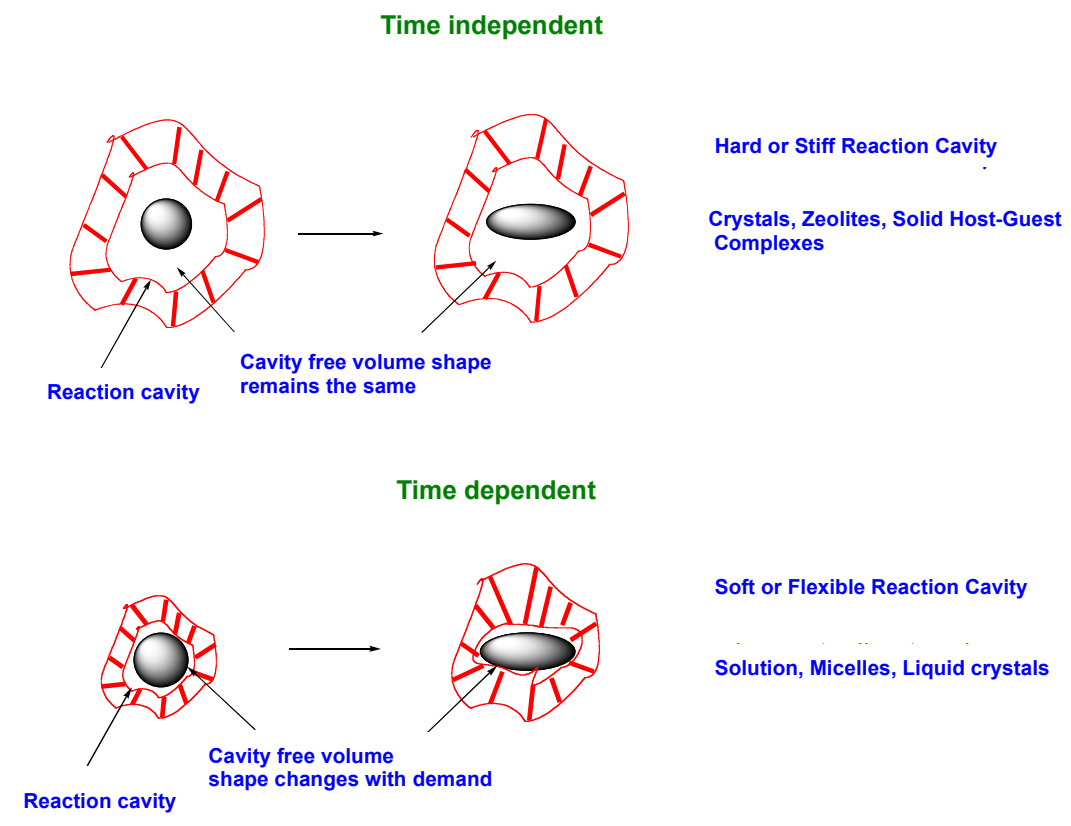

Figure 18 Cartoon representation of importance of free volume around a reactant in a reaction cavity. Two types of reaction cavity (stiff and flexible) are shown. Note: in the latter the extent of free volume changes with the demand. 

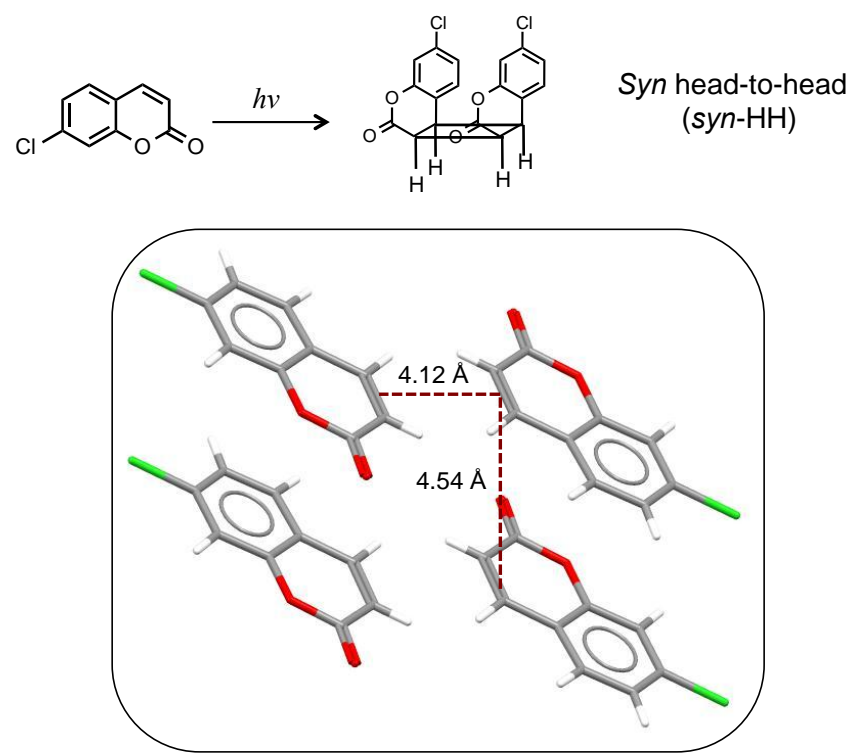

Figure 19 Irradiation of crystalline 7-chlorocoumarin yields a single dimer (syn head-tohead). The packing arrangement of 7-chlorocoumarin is shown in the figure below. 

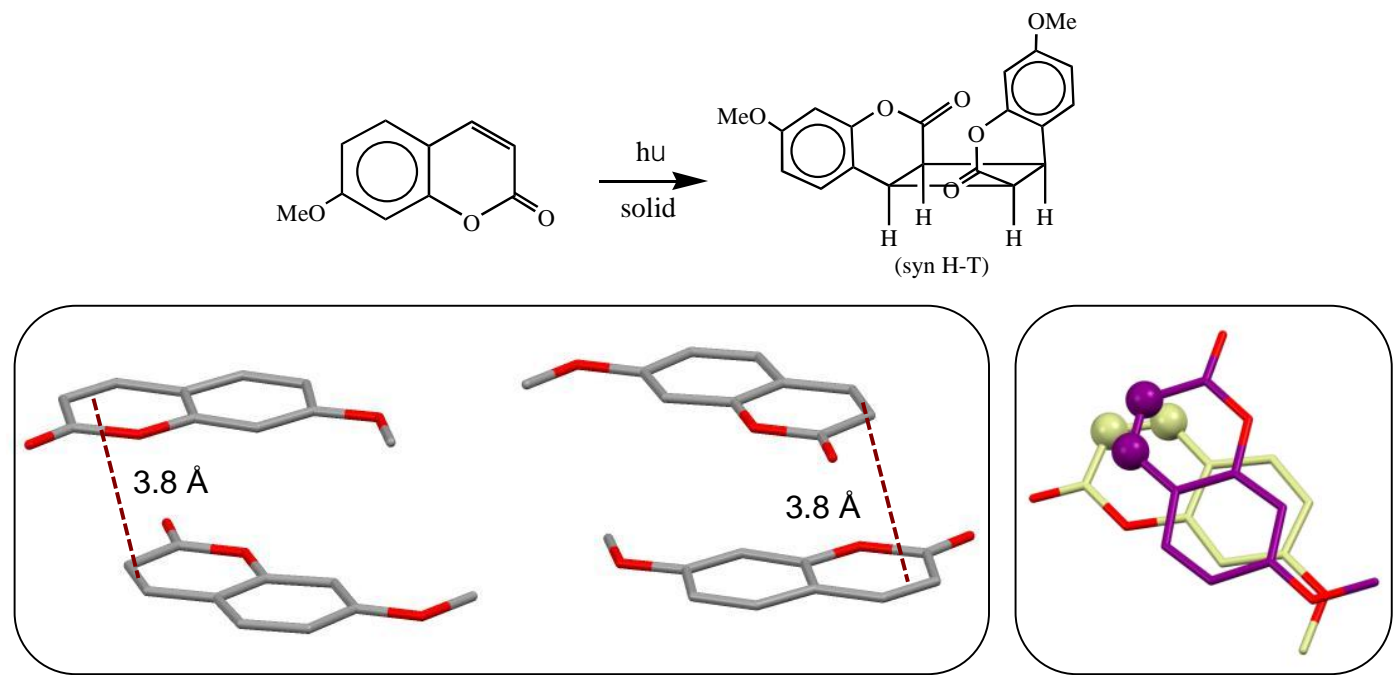

Figure 20 Irradiation of crystalline 7-methoxycoumarin yields syn head-to-tail dimer. In the figure below the packing arrangement of two molecules of 7-methoxycoumarin is shown. The reactive double bonds are rotated by $65^{\circ}$. 


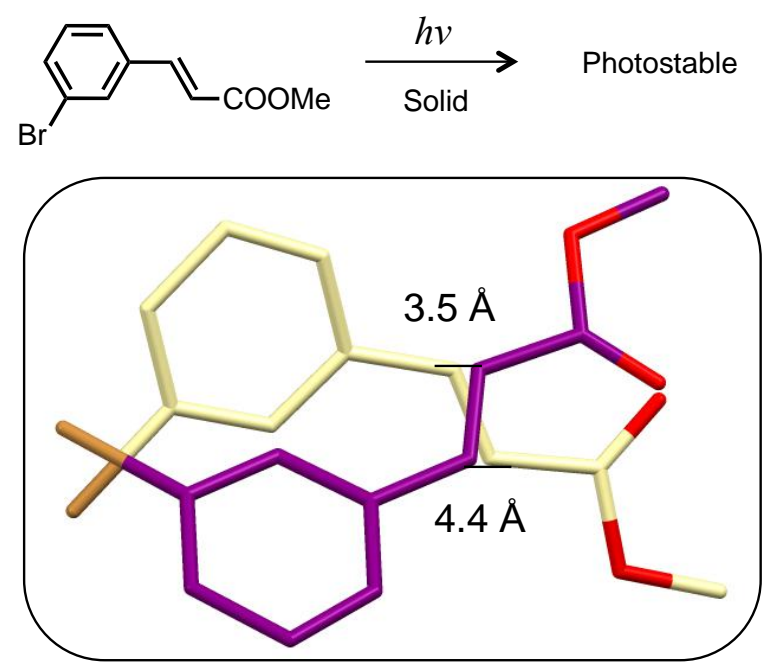

Figure 21 Methyl $m$-bromocinnamate is photostable in the crystalline state. In the figure below the packing arrangement of two molecules of methyl $m$-bromocinnamate is shown. The reactive double bonds rotated by $28^{\circ}$. 
Passive Reaction Cavity

Guest-cavity interactions very weak and non-directional

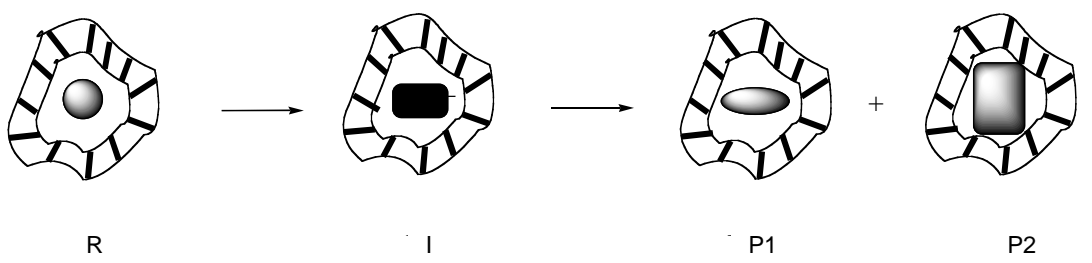

Active Reaction Cavity

Guest-cavity interactions strong and directional

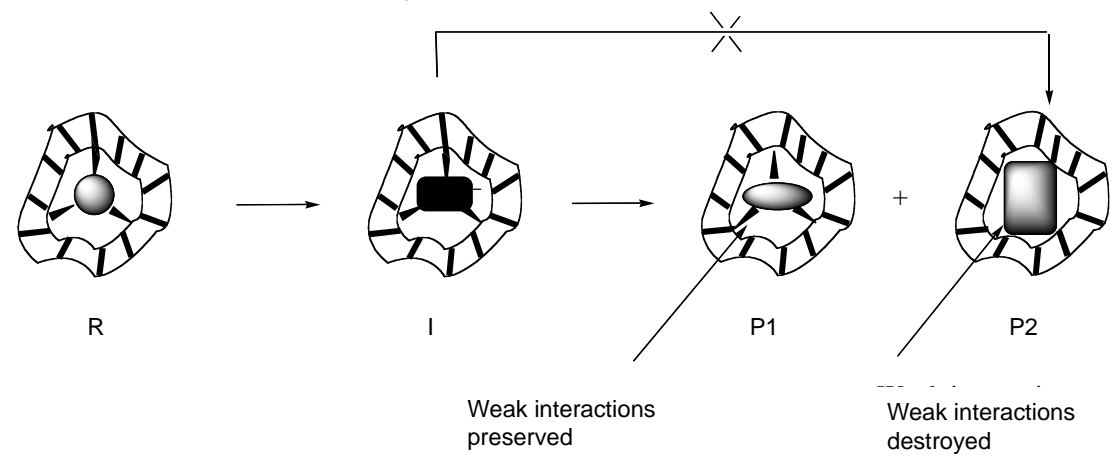

Figure 22 A cartoon representation of two types of reaction cavities, passive and active.

In the passive reaction cavity there is no interaction between the reactant and the cavity whereas in the active reaction cavity there are weak interactions between the reactant and the cavity wall. 


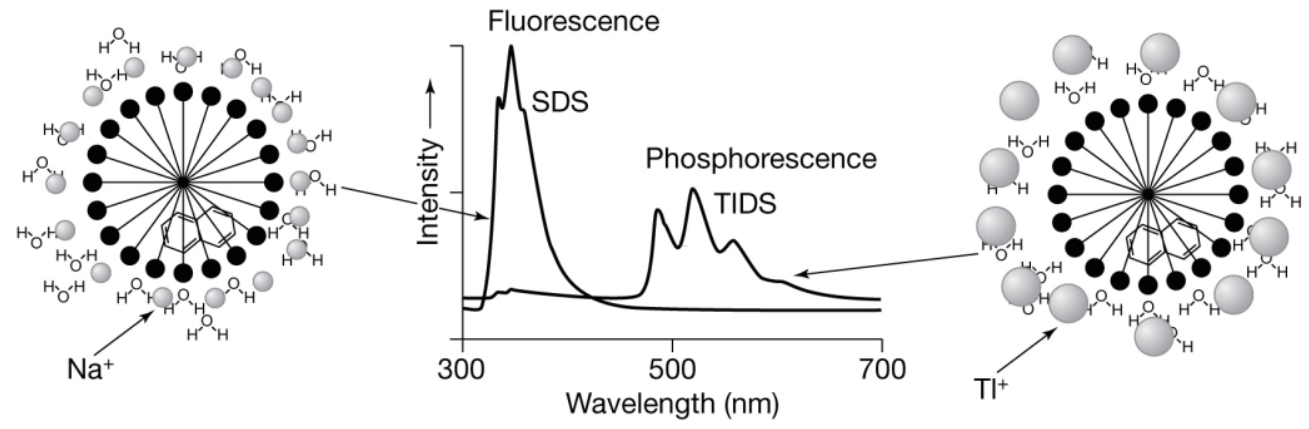

Figure 23 Emission spectra of naphthalene in $\mathrm{Na}^{+}$and $\mathrm{Tl}^{+}$dodecyl sulfate micelle at room temperature. Note: in $\mathrm{Na}^{+}$micelle only fluorescence and in $\mathrm{Tl}^{+}$micelle only phosphorescence is seen. 


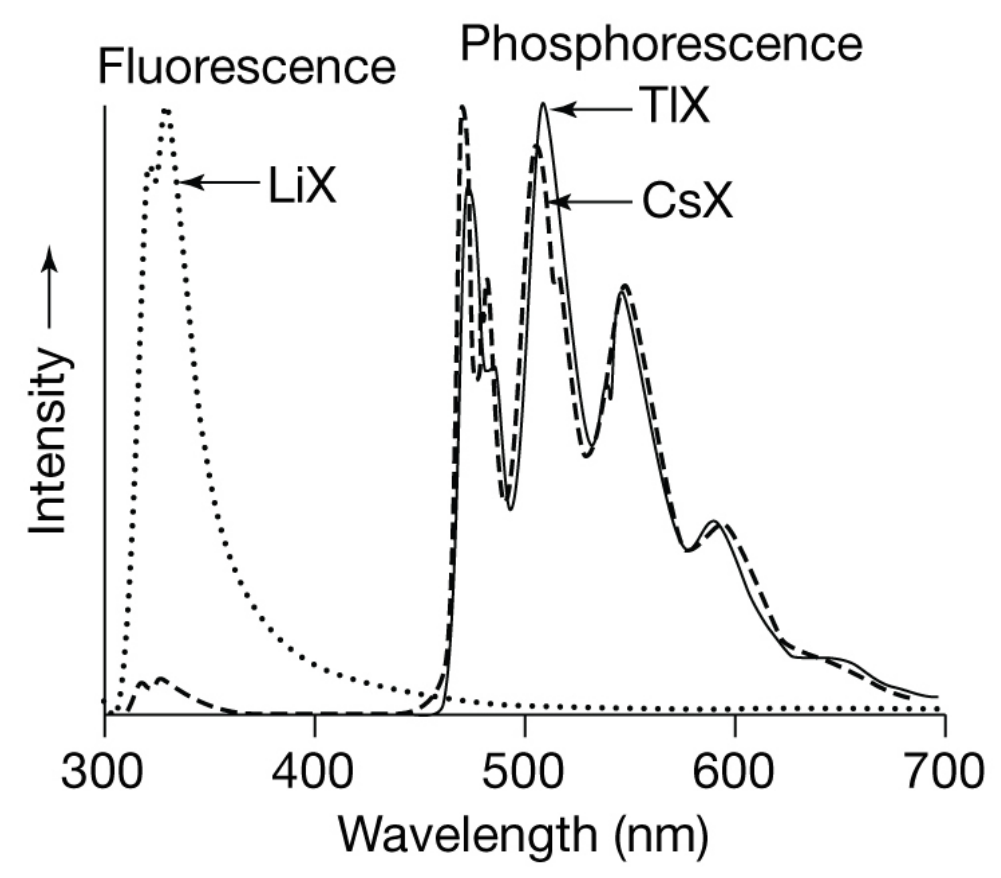

Figure 24 Emission spectra of naphthalene included in the MX-type zeolites where $\mathrm{M}=$ $\mathrm{Li}^{+}, \mathrm{Cs}^{+}$, or $\mathrm{Tl}^{+}$. Note: in $\mathrm{Li}^{+}$zeolite only fluorescence and in $\mathrm{Tl}^{+}$zeolite only phosphorescence is seen. In $\mathrm{Cs}^{+}$zeolite a mixture of fluorescence and phosphorescence is seen. 


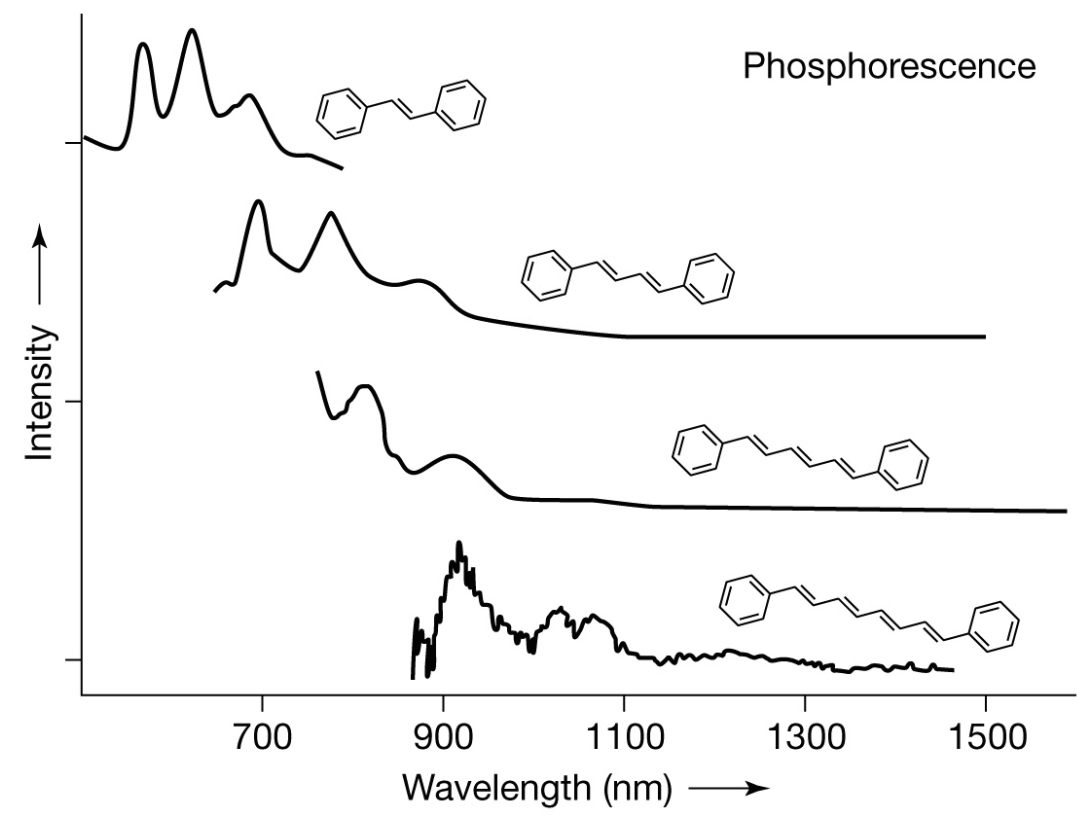

Figure 25 Phosphorescence at room temperature from trans-stilbene, all trans-1,4diphenylbutadiende, all-trans-1,6-diphenylhexatriene and all-trans-1,8diphenyloctatetraene when included in with $\mathrm{Tl}^{+}$exchanged $\mathrm{X}$ zeolites. 


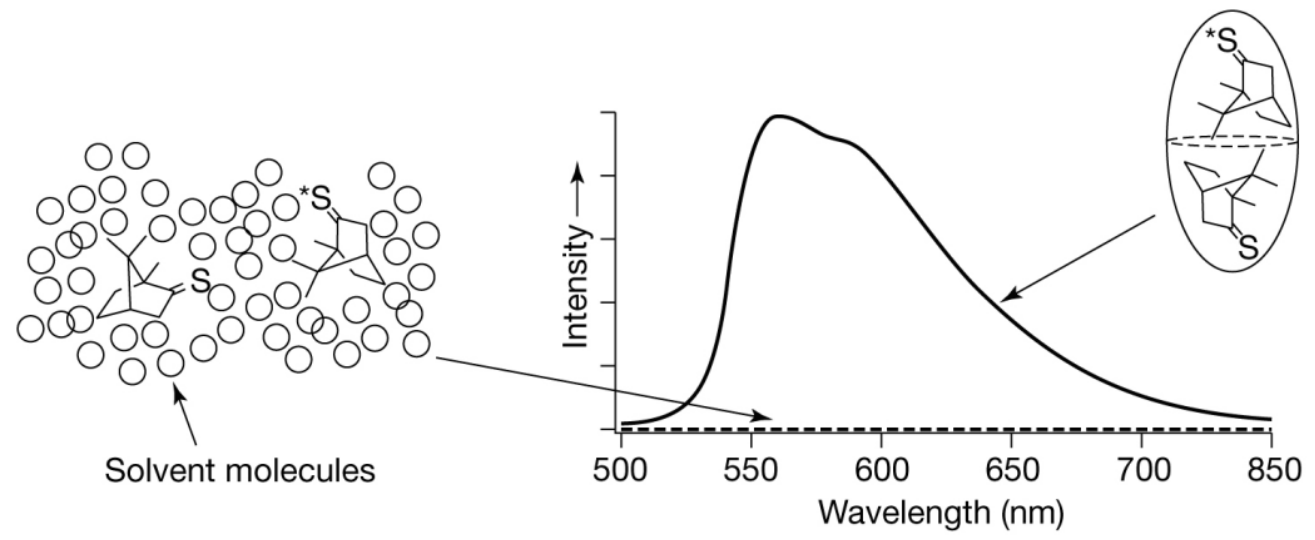

Figure 26 Phosphorescence from thiocamphor included within octa acid in water (solid line) and perfluorodimethylcyclohexane solvent (dotted line) at room temperature. 
a) Chiral Inductor Method (CIM)

$\triangle \quad$ Achiral Reactant $(\mathrm{R})$

Chiral Inductor (CI)

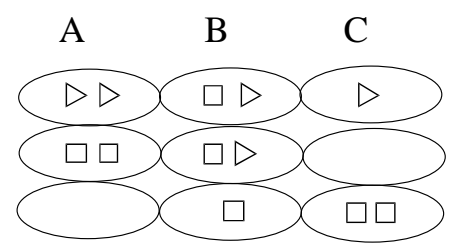

$\mathrm{A}, \mathrm{C}=0 \%$ ee

$\mathrm{B}=0-100 \%$ ee

$\mathrm{D}, \mathrm{E}, \mathrm{F}=$ No reaction

F $\quad$ E $\quad D$

b) Chiral Auxiliary Method (CAM)

Achiral Reactant $\begin{gathered}\text { Covalent bond } \\ \text { Chiral Auxiliary }\end{gathered}$

c) [Chiral Inductor + Chiral Auxiliary Method] (CIAM)
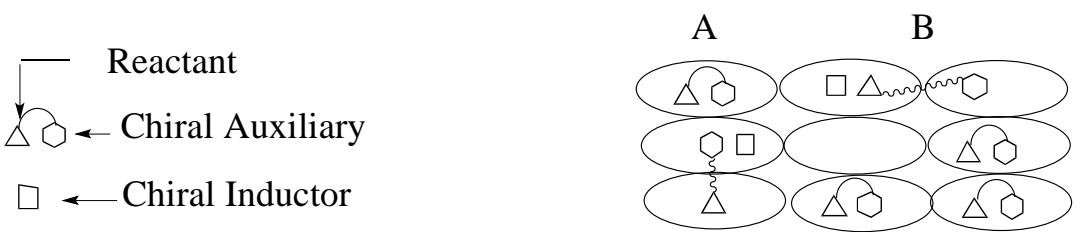

Figure 27 Distributions of reactant and chiral inductor molecules in zeolite cages. 

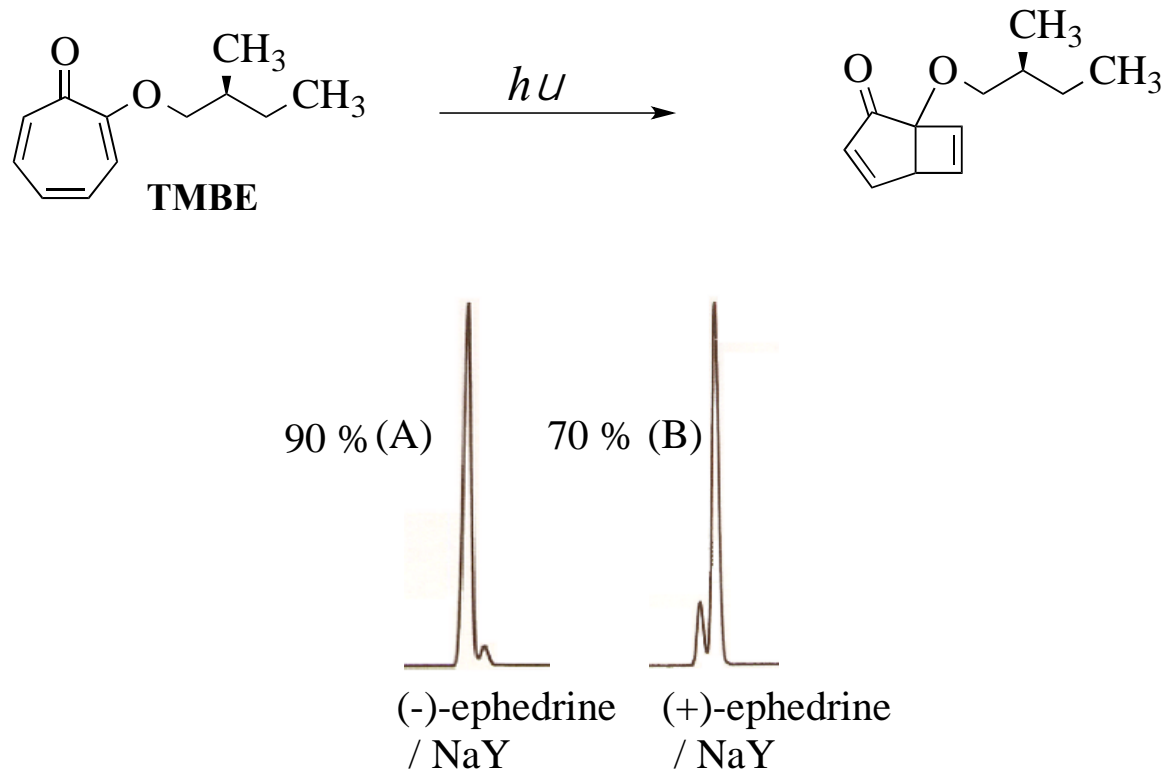

Figure 28 Results on the photocyclization of $(S)$-tropolone 2-methylbutyl ether within a chirally modified $\mathrm{NaY}$ zeolite. The \% diastereomeric excess and the isomer enhanced are shown on the GC traces. Note that the extent of chiral induction brought forth by the antipodes is not the same with achiral and chiral tropolone ether. 


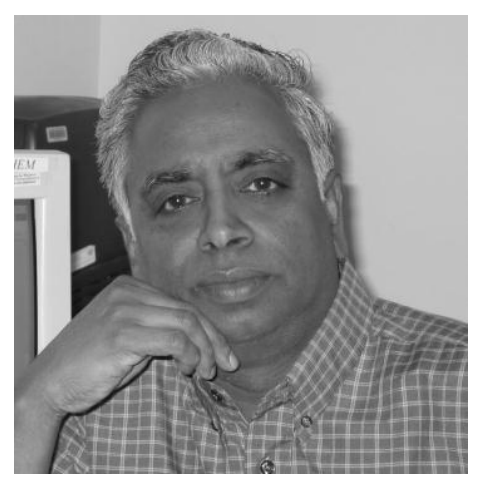

V. Ramamurthy is Professor of Chemistry at University of Miami, Coral Gables, Florida. The author of over 375 publications, editor of fourteen monographs (1991-2011), guest editor of six special journal issues (1987-2014), coauthor of a recently (2010) published textbook Modern Molecular Photochemistry of Organic Molecules and Senior Editor of Langmuir, he received his $\mathrm{Ph}$. D. from the University of Hawaii, Honolulu, USA (R. S. H. Liu), and pursued his postdoctoral training at the University of Western Ontario, London, Canada (P. de Mayo) and Columbia University, New York, USA (N. J. Turro). Before joining the University of Miami in 2005, he was Bernard-Baus Professor of Chemistry at Tulane University, New Orleans, Louisiana, Senior Scientist at the Du Pont Company in Wilmington, Delaware, and Associate Professor at the Indian Institute of Science, Bangalore, India. His research interests are in the area of supramolecular photochemistry that encompasses photochemistry in crystals, zeolites, cavitands and capsules.

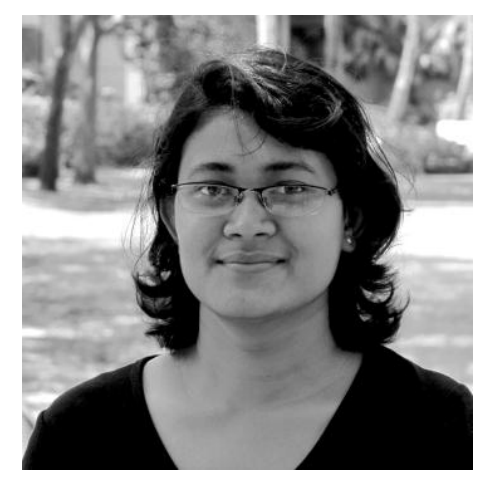

Barnali Mondal received her Ph. D at University of Miami, under the supervision of Prof. V. Ramamurthy in December, 2014 for her thesis in the area of 'Supramolecular Photochemistry'. Prior to joining University of Miami, she received her B.Sc (2006) and M.Sc (2008) from Presidency College, Kolkata. Her research at Miami focused on solid-state photochemistry and use of supramolecular host systems in stabilizing nanoparticles in aqueous solution. She is continuing in the laboratory of Prof. Ramamurthy as a post-doctoral fellow. 